TRANSACTIONS OF THE

AMERICAN MATHEMATICAL SOCIETY

Volume 364, Number 1, January 2012, Pages 309-360

S 0002-9947(2011)05399-7

Article electronically published on August 2, 2011

\title{
GRAPH BRAID GROUPS AND RIGHT-ANGLED ARTIN GROUPS
}

\author{
JEE HYOUN KIM, KI HYOUNG KO, AND HYO WON PARK
}

\begin{abstract}
We give a necessary and sufficient condition for a graph to have a right-angled Artin group as its braid group for braid index $\geq 5$. In order to have the necessity part, graphs are organized into small classes so that one of the homological or cohomological characteristics of right-angled Artin groups can be applied. Finally we show that a given graph is planar iff the first homology of its 2-braid group is torsion-free, and we leave the corresponding statement for $n$-braid groups as a conjecture along with a few other conjectures about graphs whose braid groups of index $\leq 4$ are right-angled Artin groups.
\end{abstract}

\section{INTRODUCTION}

As motivated by robotics, graph braid groups were first introduced by Ghrist 12 in 1998. A motion of $n$ robots is a one-parameter family of $n$-tuples in a graph $\Gamma$ that are pairwise distinct, that is, a path in the configuration space of $\Gamma$ which is the complement of diagonals in the $n$-fold product $\Gamma^{n}$. The graph $n$-braid group over $\Gamma$ is, roughly speaking, the group of these motions under concatenation and is more precisely the fundamental group of the (unordered) configuration space.

On the other hand, a right-angled Artin group is a group that has a finite presentation each of whose relators is a commutator of generators. It was sometimes called a graph group since its presentation can be defined via a graph whose vertices are generators and each of whose edges gives a relator that is the commutator of two ends. The survey article [4 by Charney contains a good overview.

Graph braid groups that can be computed directly by hand usually have presentations whose relators are all in the form of a commutator that is not however necessarily a commutator of generators. In fact, all graph braid groups were presumed to be right-angled Artin groups until it was shown by Abrams and Ghrist in 2 that the pure 2-braid groups of the complete graph $K_{5}$ and the complete bipartite graph $K_{3,3}$ are surface groups, so they are not right-angled Artin groups. Since then, it was a reasonable conjecture that every planar-graph braid group is a right-angled Artin group. Connelly and Doig in [6] proved that every lineartree braid group is a right-angled Artin group where a tree is linear if it does not contain $T_{0}$ in Figure 1(a). Meanwhile, Crisp and Wiest in [7] proved that every

Received by the editors August 11, 2009 and, in revised form, May 24, 2010, June 6, 2010 and June 12, 2010.

2010 Mathematics Subject Classification. Primary 20F36, 20F65, 57M15.

Key words and phrases. Braid group, right-angled Artin group, discrete Morse theory, planar, graph.

This work was supported by the Korea Science and Engineering Foundation (KOSEF) grant funded by the Korea government (MOST) (No. R01-2006-000-10152-0).

(C)2011 American Mathematical Society 309

Reverts to public domain 28 years from publication 
graph braid group embeds in a right-angled Artin group. Conversely, Sabalka in 14 proved that every right-angled Artin group can be realized as a subgroup of a graph braid group. Farley and Sabalka in [9] described an efficient method to obtain a presentation for any graph braid group using the discrete Morse theory.

Recently, Farley and Sabalka in [10] characterized trees whose braid groups are right-angled Artin groups. They proved that for braid indices 2 or 3 , tree braid groups are always right-angled Artin groups, and for braid index $\geq 4$, a braid group over a given tree is a right-angled Artin group if and only if the tree does not contain $T_{0}$. In this article, this result is generalized to arbitrary graphs for braid index $\geq 5$. In fact, we will prove the following two theorems:

Theorem A. If a graph $\Gamma$ contains neither $T_{0}$ nor $S_{0}$ in Figure 1, then the graph $n$-braid group $B_{n} \Gamma$ is a right-angled Artin group for braid index $n \geq 5$.

Theorem B. If a graph $\Gamma$ contains $T_{0}$ or $S_{0}$ in Figure 1, then the graph $n$-braid group $B_{n} \Gamma$ is not a right-angled Artin group for braid index $n \geq 5$.

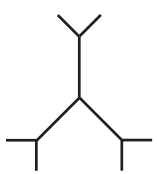

(a) $T_{0}$

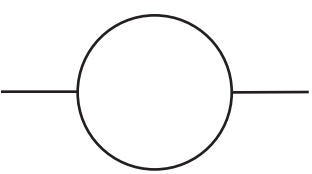

(b) $S_{0}$

FiguRe 1. $T_{0}$ and $S_{0}$

This paper is organized as follows. In $\S 2$, we explain how to simplify configuration spaces using the discrete Morse theory. Basic constructions and notation introduced will be used throughout the article. As applications, we obtain a presentation of $B_{n} \Gamma$ for any graph $\Gamma$ containing neither $T_{0}$ nor $S_{0}$ and prove Theorem A.

For graphs $\Gamma$ and $\Gamma^{\prime}$ and $n \geq n^{\prime}$, suppose that there is an embedding $i: \Gamma^{\prime} \rightarrow \Gamma$ of graphs that induces a surjection $i^{*}: H^{*}\left(B_{n} \Gamma ; \mathbb{Z}_{2}\right) \rightarrow H^{*}\left(B_{n^{\prime}} \Gamma^{\prime} ; \mathbb{Z}_{2}\right)$ of cohomology algebras whose kernel is generated by homogeneous classes of degree 1 and 2, and moreover, suppose that $B_{n^{\prime}} \Gamma^{\prime}$ is not a right-angled Artin group. Then by combining results by Charney-Davis [6] and by Farley-Sabalka [10, one can conclude that $B_{n} \Gamma$ is not a right-angled Artin group either. This argument was introduced by Farley and Sabalka in 10 to show that an $n$-braid group over a tree containing $T_{0}$ is not a right-angled Artin group for $n \geq 4$. One of the hypotheses of this argument does not hold if there is no embedding $i: \Gamma^{\prime} \rightarrow \Gamma$ inducing an injection $i_{*}: H_{1}\left(B_{n^{\prime}} \Gamma^{\prime}\right) \rightarrow H_{1}\left(B_{n} \Gamma\right)$. Furthermore, if $\Gamma^{\prime}$ has no vertices of valency 1 , there is no room to accommodate extra punctures when we construct an embedding $i: \Gamma^{\prime} \rightarrow \Gamma$ to obtain some result for $B_{n} \Gamma$ for all braid indices $n \geq 5$. In $\S 3$, we circumvent these difficulties by dividing the class of graphs containing $T_{0}$ but not $S_{0}$ into smaller classes, for each of which the above argument is applicable. So Theorem B is proved for a graph $\Gamma$ that contains $T_{0}$ but not $S_{0}$.

Given a finite presentation of a group $G$, let $F$ be the free group over generators and $R$ be the normal subgroup generated by relators. Suppose $R \subset[F, F]$. Then the inclusion induces a homomorphism $\Phi_{G}: R /[F, R] \rightarrow[F, F] /[F,[F, F]]$ which can be regarded as the dual of the cup product $H^{1}(G) \wedge H^{1}(G) \rightarrow H^{2}(G)$ according to Matei-Suciu [13. In $\S 4$, we derive and use a number of necessary conditions for $G$ to 
be a right-angled Artin group. For example, if $G$ is a right-angled Artin group, $\Phi_{G}$ is injective, which we employ to show that $B_{n} S_{0}$ and $B_{n} \Theta$ are not right-angled Artin groups where $\Theta$ is the graph of the figurative shape $\Theta$. If $G$ is a right-angled Artin group, the cohomology ring $H^{*}(G)$ is completely determined by $\Phi_{G}$ and $H_{1}(G)$, and this fact also provides a useful obstruction for $G$ to be a right-angled Artin group. A more sophisticated application of this idea is to consider homomorphisms $h_{*}$ : $R^{\prime} /\left[F^{\prime}, R^{\prime}\right] \rightarrow R /[F, R]$ and $\bar{h}:\left[F^{\prime}, F^{\prime}\right] /\left[F^{\prime},\left[F^{\prime}, F^{\prime}\right]\right] \rightarrow[F, F] /[F,[F, F]]$ induced from an embedding $\Gamma^{\prime} \rightarrow \Gamma$. Here we assume that $R \subset[F, F]$ and $R^{\prime} \subset\left[F^{\prime}, F^{\prime}\right]$ for $B_{n} \Gamma=F / R$ and $B_{n} \Gamma^{\prime}=F^{\prime} / R^{\prime}$. If we can directly show from their configuration spaces that $\Phi_{B_{n} \Gamma^{\prime}}$ is not injective and $h_{*}: H_{2}\left(B_{n} \Gamma^{\prime}\right) \rightarrow H_{2}\left(B_{n} \Gamma\right)$ is injective, then the commutativity $\Phi_{B_{n} \Gamma} h_{*}=\bar{h} \Phi_{B_{n} \Gamma^{\prime}}$ implies that $\Phi_{B_{n} \Gamma}$ is not injective and so $B_{n} \Gamma$ is not a right-angled Artin group. Using this argument, we prove Theorem B for a planar graph $\Gamma$ containing $S_{0}$. For non-planar graphs, we prove Theorem B by showing that the first homology of its braid group has a 2-torsion.

We conclude the paper with four conjectures. Two of them are about extending our main results in this paper to graph braid groups of braid index $\leq 4$. The other two conjectures are characterizations of planar graphs via graph braid groups. One is that graph braid groups of planar graphs are commutator-related, and the other is that the first homology group of their graph braid groups are torsion-free. As mentioned above, a given graph must be planar if the first homology of its graph braid group is torsion-free. Finally we prove the converse when the braid index is 2 .

\section{Discrete Configuration SPaCes And their Morse COMPlexes}

2.1. Discrete configuration spaces. Let $\Gamma$ be a connected finite graph. We may regard $\Gamma$ as a metric space by treating each edge as the unit interval $[0,1]$. The topological configuration space of $\Gamma$ is defined by

$$
C_{n} \Gamma=\left\{\left(x_{1}, \ldots, x_{n}\right) \in \Gamma^{n} \mid x_{i} \neq x_{j} \text { if } i \neq j\right\} .
$$

The unordered topological configuration space of $\Gamma$ is defined by $U C_{n} \Gamma=C_{n} \Gamma / S_{n}$, where the symmetric group $S_{n}$ acts on $C_{n} \Gamma$ by permuting coordinates or is alternatively defined by

$$
U C_{n} \Gamma=\left\{\left\{x_{1}, \ldots, x_{n}\right\} \subset \Gamma \mid x_{i} \neq x_{j} \text { if } i \neq j\right\} .
$$

The topological configuration spaces are open complexes, and it is hard to make them finite complexes. We study the following alternative.

We now regard a graph $\Gamma$ as a 1-dimensional CW complex. Then the $n$-fold Cartesian product $\Gamma^{n}$ of $\Gamma$ has a CW complex structure and a cell in $\Gamma^{n}$ has the form $\left(\sigma_{1}, \ldots, \sigma_{n}\right)$, where each $\sigma_{i}$ is either a vertex or an edge of $\Gamma$. Let $\partial \sigma$ denote the set of end vertices if $\sigma$ is an edge, or $\sigma$ itself if $\sigma$ is a vertex. The ordered discrete configuration space of $\Gamma$ is defined by

$$
D_{n} \Gamma=\left\{\left(\sigma_{1}, \ldots, \sigma_{n}\right) \in \Gamma^{n} \mid \partial \sigma_{i} \cap \partial \sigma_{j}=\emptyset \text { if } i \neq j\right\} .
$$

The unordered discrete configuration space of $\Gamma$ is defined by $U D_{n} \Gamma=D_{n} \Gamma / S_{n}$ or is alternatively defined by

$$
U D_{n} \Gamma=\left\{\left\{\sigma_{1}, \ldots, \sigma_{n}\right\} \subset \Gamma \mid \partial \sigma_{i} \cap \partial \sigma_{j}=\emptyset \text { if } i \neq j\right\} .
$$

Abrams showed in [1] that for any $n>1$ and any graph $\Gamma$ with at least $n$ vertices, $D_{n} \Gamma\left(U D_{n} \Gamma\right.$, respectively) is a deformation retract of the topological ordered 
(unordered, respectively) configuration space if a graph is sufficiently subdivided so that

(1) Each path between two vertices of valency $\neq 2$ passes through at least $n+1$ edges.

(2) Each loop from a vertex to itself which cannot be shrunk to a point in $\Gamma$ passes through at least $n+1$ edges.

Abrams conjectured the version where there are at least $n-1$ edges in the condition (1) instead of $n+1$ edges. He also observed that the statement is false if there are fewer than $n-1$ edges. For the sake of completeness, we will prove the conjecture in Theorem 2.4 ,

From now on, we assume that $\Gamma$ is always sufficiently subdivided so that the discrete configuration spaces are deformation retracts of their topological counterparts. The pure graph braid group $P_{n} \Gamma$ and the graph braid group $B_{n} \Gamma$ of $\Gamma$ are the fundamental groups of the ordered and the unordered discrete configuration spaces of $\Gamma$, that is,

$$
P_{n} \Gamma=\pi_{1}\left(D_{n} \Gamma\right) \quad \text { and } \quad B_{n} \Gamma=\pi_{1}\left(U D_{n} \Gamma\right) .
$$

Abrams showed in [1] that discrete configuration spaces $D_{n} \Gamma$ and $U D_{n} \Gamma$ are cubical complexes of non-positive curvature and so are locally $\mathrm{CAT}(0)$ spaces. In particular, $D_{n} \Gamma$ and $U D_{n} \Gamma$ are Eilenberg-MacLane spaces, and $P_{n} \Gamma$ and $B_{n} \Gamma$ are torsion-free, and the word problems and the conjugacy problems in $P_{n} \Gamma$ and $B_{n} \Gamma$ are solvable.

2.2. Presentation of graph braid groups. In order to compute a presentation of graph braid groups, Farley and Sabalka in [9] considered a complex that is as simple as possible but is still homotopy equivalent to $U D_{n} \Gamma$. By using the discrete Morse theory developed by Forman in [11, they proved that $U D_{n} \Gamma$ can be systemically collapsed to a complex, called a Morse complex, which is simple enough to compute $\pi_{1}$. Mostly following [9], we quickly review how to collapse $U D_{n} \Gamma$ to its Morse complex.

Step I. Give an order of vertices of a graph $\Gamma$. First choose a maximal tree $T$ in $\Gamma$. We also assume that $T$ is always sufficiently subdivided in the sense of 2.1. Edges in $\Gamma-T$ are called deleted edges. Pick a vertex of valency 1 in $T$ to be a basepoint and assign 0 to this vertex. Let $R$ be a regular neighborhood of $T$ in a plane. Then the boundary $\partial R$ is a simple closed curve in the plane. Starting from the base vertex 0 , we number unnumbered vertices of $T$ as we travel along $\partial R$ clockwise. Figure 2(a) illustrates this procedure for the graph $S_{0}$ and for $n=4$. There is one deleted edge $d$ to form a maximal tree. All vertices in $\Gamma$ are numbered and so are referred to by the non-negative integers.

Each edge $e$ in $T$ is oriented so that the initial vertex $\iota(e)$ is larger than the terminal vertex $\tau(e)$. The edge $e$ is denoted by $\tau(e)-\iota(e)$. A (open, cubical) cell $c$ in the unordered configuration space $U D_{n} \Gamma$ can be written as $\left\{c_{1}, \ldots, c_{n}\right\}$, where each $c_{j}$ is either a vertex or an edge in $\Gamma$. The cell $c$ is an $i$-cell if the number of edges among $c_{j}$ 's is $i$. For example, $\{6-10, d, 0-1,5\}$ in Figure 2 represents a 3 -cell in $U D_{4} S_{0}$. Let $K_{i}$ denote the set of all $i$-cells of $U D_{n} \Gamma$.

Step II. Define a function $W: K_{i} \rightarrow K_{i+1} \cup\{$ void $\}$ for $i \geq-1$. For a cell $c=\left\{c_{1}, \ldots, c_{n-1}, v\right\} \in U D_{n} \Gamma$, a vertex $v$ is called blocked in $c$ if $\tau(e)$ appears in $c$ (as a vertex or as an end vertex of an edge) for the edge $e$ with $\iota(e)=v$. 


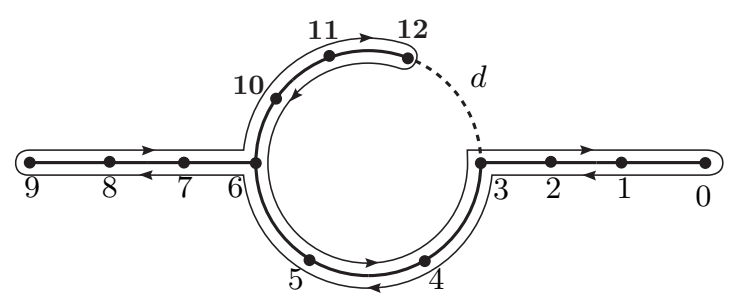

(a) Numbering a maximal tree $T$ of $S_{0}$ for $n=4$

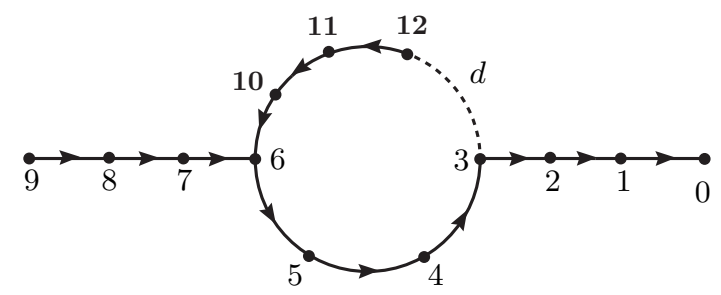

(b) Orientations of edges in a maximal tree $T$ of $S_{0}$

Figure 2. Maximal tree of $S_{0}$

Let $K_{-1}=\emptyset$. Define $W$ by induction on $i$. Let $c=\left\{c_{1}, c_{2}, \ldots, c_{n}\right\}$ be an $i$-cell. If $c \notin \operatorname{im}(W)$ and there are unblocked vertices in $c$ and, say, $c_{1}$ is the smallest unblocked vertex, then $W(c)=\left\{v-c_{1}, c_{2}, \ldots, c_{n}\right\}$, where $v$ - $c_{1}$ is the edge in $T$ such that $v<c_{1}$. Otherwise, $W(c)=$ void. Then it is not hard to see that $W$ is welldefined and each cell in $W\left(K_{i}\right)-$ void $\}$ has the unique preimage under $W$, and there is no cell in $K_{i}$ that is both an image and a preimage of other cells under $W$.

Step III. Collapse $U D_{n} \Gamma$ to a Morse complex. For each pair $(c, W(c)) \in$ $K_{i} \times\left(W\left(K_{i}\right)-\{\operatorname{void}\}\right)$, we homotopically collapse the closure $\overline{W(c)}$ onto $\overline{W(c)}-$ $(W(c) \cup c)$ to obtain a Morse complex of $U D_{n} \Gamma$.

For a pair $(c, W(c)) \in K_{i} \times\left(W\left(K_{i}\right)-\{\operatorname{void}\}\right)$, cells $c$ and $W(c)$ are said to be redundant and collapsible, respectively. Redundant or collapsible cells disappear in a Morse complex. A cell that survives in a Morse complex is said to be critical. Every cell in $\Gamma$ is one of these three kinds that can also be characterized as follows: An edge $e$ in a cell $c=\left\{c_{1}, \ldots, c_{n-1}, e\right\}$ is order-respecting if $e$ is not a deleted edge and there is no vertex $v$ in $c$ such that $v$ is adjacent to $\tau(e)$ and $\tau(e)<v<\iota(e)$. A cell is critical if it contains neither order-respecting edges nor unblocked vertices. A cell is collapsible if it contains at least one order-respecting edge and each unblocked vertex is larger than the initial vertex of some order-respecting edge. A cell is redundant if it contains at least one unblocked vertex that is smaller than the initial vertices of all order-respecting edges. Notice that there is exactly one critical 0 -cell $\{0,1, \ldots, n-1\}$.

For example, consider $U D_{4} S_{0}$ in Figure 2 . The cells $\{0,1,2,3\}$ and $\{6-10,7,8, d\}$ are critical, the cells $\{1-2,5,6, d\}$ and $\{1-2,5,6-10,7\}$ are collapsible, and the cells $\{8,6-10,11, d\}$ and $\{1,2-3,5,6-10\}$ are redundant. 
Example 2.1. Morse complex of $U D_{2} S_{0}$.

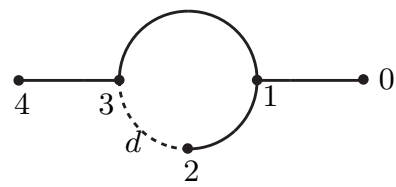

(a) Maximal tree of $S_{0}$ for $n=2$

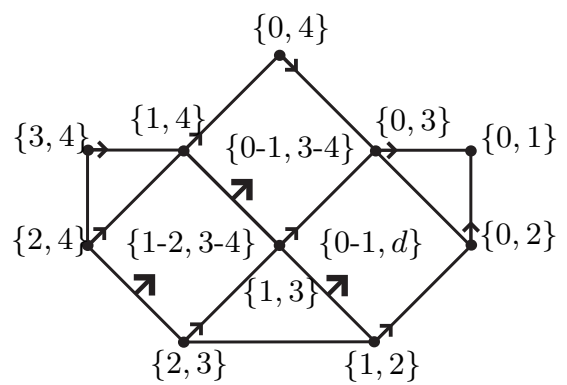

(b) $U D_{2} S_{0}$ and gradient field for collapsing

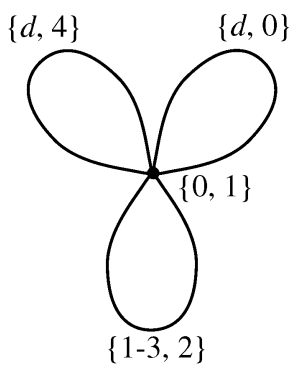

(c) Morse complex

FiguRE $3 . S_{0}$

The complex $U D_{2} S_{0}$ is a 2-dimensional complex as in Figure 3(b). The order of vertices of a maximal tree of $S_{0}$ as in Figure 3(a) determines $W$ as follows: For 0-cells, $W(\{0,2\})=\{0,1-2\}, W(\{0,3\})=\{0,1-3\}, W(\{0,4\})=\{0,3-4\}$, $W(\{1,2\})=\{0-1,2\}, W(\{1,3\})=\{0-1,3\}, W(\{1,4\})=\{0-1,4\}, W(\{2,3\})=$ $\{1-2,3\}, W(\{2,4\})=\{1-2,4\}, W(\{3,4\})=\{1-3,4\}$, and for 1-cells, $W(\{3-4,2\})=$ $\{1-2,3-4\}, W(\{d, 1\})=\{0-1, d\}, W(\{3-4,1\})=\{0-1,3-4\}$.

After collapsing, a Morse complex of $U D_{2} S_{0}$ is a 1-dimensional complex which has one 0 -cell $\{0,1\}$ and three 1-cells $\{0, d\},\{4, d\},\{1-3,2\}$ as depicted in Figure 3 (c).

The braid group $B_{n} \Gamma$ is now given by the fundamental group of a Morse complex of $U D_{n} \Gamma$. Thus $B_{n} \Gamma$ has a presentation whose generators are critical 1-cells and whose relators are boundary words of critical 2-cells in terms of critical 1-cells. For example, $B_{2} S_{0}$ is the free group generated by the three critical 1-cells above.

In order to rewrite a word in 1-cells of $U D_{n} \Gamma$ into a new word in critical 1-cells, we use the rewriting homomorphism $\tilde{r}$ from the free group on $K_{1}$ to the free group on the set of critical 1-cells defined as follows: First define a homomorphism $r$ from the free group on $K_{1}$ to itself by $r(c)=1$ if $c$ is collapsible, $r(c)=c$ if $c$ is critical, and

$$
r(c)=\left\{v-v_{1}, v_{2}, \ldots, v_{n-1}, \iota(e)\right\}\left\{v, v_{2}, \ldots, v_{n-1}, e\right\}\left\{v-v_{1}, v_{2}, \ldots, v_{n-1}, \tau(e)\right\}^{-1}
$$

if $c=\left\{v_{1}, v_{2}, \ldots, v_{n-1}, e\right\}$ is redundant such that $v_{1}$ is the smallest unblocked vertex and $e$ is the edge in $c$. Forman's discrete Morse theory in [11 guarantees that there is a non-negative integer $k$ such that $r^{k}(c)=r^{k+1}(c)$ for all $c \in K_{1}$. An essential idea is that a sequence of consecutive collapsing never circles back like a gradient flow of a height function. Once this fact is established, the finiteness of $K_{1}$ implies the existence of such a $k$. Let $\tilde{r}=r^{k}$; then for any $c \in K_{1}, \tilde{r}(c)$ is a word in critical 1-cells that is the image of $c$ under the quotient map defined by collapsing $U D_{n} \Gamma$ 
unto its Morse complex. We note that $k=0$ iff $c$ is critical, $k=1$ iff $c$ is collapsible, and $k \geq 2$ iff $c$ is redundant. In fact, it is impossible that $r(c)$ is a product of three critical cells, and so $k>1$ if $c$ is redundant.

Using the rewriting map $\tilde{r}$, we can rewrite the boundary word of a critical 2-cell in terms of critical 1-cells. Thus it is possible to compute a presentation of $B_{n} \Gamma$ using a Morse complex of $U D_{n} \Gamma$. However, the computation of $\tilde{r}$ is usually tedious, and the following lemma somewhat shortens it. In particular, the lemma will be useful in the proof of Theorem 2.4 .

Lemma 2.2. Let $c$ be a 1-cell in $U D_{n} \Gamma$ and $e$ be an edge in $\Gamma$ such that $\iota(e)$ is an unblocked vertex in $c$. If $\iota(e)-\tau(e)=1$, then $\tilde{r}(c)=\tilde{r}\left(V_{e}(c)\right)$, where $V_{e}(c)$ denotes the 1-cell obtained from $c$ by replacing $\iota(e)$ with $\tau(e)$.

Proof. We first remark that this result was proved by Farley and Sabalka in 9] if $\iota(e)$ is the smallest unblocked vertex in $c$. We proceed by induction on the minimal integer $k$ such that $r^{k}(c)=r^{k+1}(c)$. Since $c$ must be either collapsible or redundant, $k \geq 1$. If $k=1$, the $c$ is collapsible and $\tilde{r}(c)=\tilde{r}\left(V_{e}(c)\right)=0$.

Assume that $k \geq 2$. Let $e^{\prime}$ be the edge in $c$ and $e_{s}$ be the edge in $\Gamma$ such that $\iota\left(e_{s}\right)$ is the smallest unblocked vertex in $c$. If $\iota(e)=\iota\left(e_{s}\right)$, then we are done by the result of Farley and Sabalka. Now assume $\iota(e) \neq \iota\left(e_{s}\right)$. Then we may write $r(c)=c_{e^{\prime}}^{L} c_{e_{s}}^{\tau}\left(c_{e^{\prime}}^{\tau}\right)^{-1}$, where $c_{e^{\prime}}^{L}$ and $c_{e^{\prime}}^{\tau}$ are 1-cells obtained from the collapsible 2-cell $W(c)$ by replacing an edge $e^{\prime}$ by $\iota\left(e^{\prime}\right)$ and $\tau\left(e^{\prime}\right)$, respectively. Since $v$ is unblocked in $c_{e^{\prime}}^{l}, c_{e_{s}}^{\tau}$ and $c_{e^{\prime}}^{\tau}$, all three 1-cells $c_{e^{\prime}}^{l}, c_{e_{s}}^{\tau}$ and $c_{e^{\prime}}^{\tau}$ are either collapsible or redundant. If $c_{e^{\prime}}^{l}$ is collapsible, then $e_{s}$ is an order-respecting edge proceeded by no unblocked vertices that are smaller than $\iota\left(e_{s}\right)$ in both $c_{e^{\prime}}^{l}$ and $V_{e}\left(c_{e^{\prime}}^{L}\right)$ by our assumption. So $V_{e}\left(c_{e^{\prime}}^{l}\right)$ is collapsible and $\tilde{r}\left(c_{e^{\prime}}^{l}\right)=\tilde{r}\left(V_{e}\left(c_{e^{\prime}}^{l}\right)\right)$. On the other hand, if $c_{e^{\prime}}^{l}$ is redundant, then $\tilde{r}\left(c_{e^{\prime}}^{l}\right)=\tilde{r}\left(V_{e}\left(c_{e^{\prime}}^{\iota}\right)\right)$ by induction because $c_{e^{\prime}}^{\iota}$ requires one less iteration of $r$ to stabilize than $c$. Similarly we can show $\tilde{r}\left(c_{e_{s}}^{\tau}\right)=\tilde{r}\left(V_{e}\left(c_{e_{s}}^{\tau}\right)\right)$ and $\tilde{r}\left(c_{e^{\prime}}^{\tau}\right)=\tilde{r}\left(V_{e}\left(c_{e^{\prime}}^{\tau}\right)\right)$. Since $\iota\left(e_{s}\right)$ is still the smallest unblocked vertex in $V_{e}(c), r\left(V_{e}(c)\right)=V_{e}\left(c_{e^{\prime}}^{l}\right) V_{e}\left(c_{e_{s}}^{\tau}\right) V_{e}\left(c_{e^{\prime}}^{\tau}\right)^{-1}$. Thus $\tilde{r}(c)=\tilde{r}(r(c))=$ $\tilde{r}\left(c_{e^{\prime}}^{l} c_{e_{s}}^{\tau}\left(c_{e^{\prime}}^{\tau}\right)^{-1}\right)=\tilde{r}\left(V_{e}\left(c_{e^{\prime}}^{L}\right) V_{e}\left(c_{e_{s}}^{\tau}\right) V_{e}\left(c_{e^{\prime}}^{\tau}\right)^{-1}\right)=\tilde{r}\left(r\left(V_{e}(c)\right)\right)=\tilde{r}\left(V_{e}(c)\right)$.

Example 2.3. $B_{4} S_{0}$ is a right-angled Artin group.

Proof. By Abrams' conjecture proved in Theorem [2.4, $B_{4} S_{0}$ is the fundamental group of $U D_{4} S_{0}$ with a subdivision, a maximal tree, and an order as given in Figure 2. Then $U D_{4} S_{0}$ has 10 critical 1-cells $\{d, 0,1,2\},\{d, 0,1,4\},\{d, 0,4,5\}$, $\{d, 4,5,6\}, \quad\{6-10,0,1,7\}, \quad\{6-10,0,7,11\}, \quad\{6-10,0,7,8\}, \quad\{6-10,7,11,12\}$, $\{6-10,7,8,11\},\{6-10,7,8,9\}$, and 4 critical 2-cells $\{d, 6-10,0,7\},\{d, 6-10,4,7\}$, $\{d, 6-10,7,11\},\{d, 6-10,7,8\}$.

The boundary word of the critical 2-cell $\{d, 6-10,0,7\}$ is the product

$$
\{6-10,0,7,12\}\{d, 0,6,7\}\{6-10,0,3,7\}^{-1}\{d, 0,7,10\}^{-1} .
$$

By Lemma 2.2, $\tilde{r}(\{d, 0,6,7\})=\tilde{r}(\{d, 0,5,7\})=\tilde{r}(\{d, 0,4,7\})=\tilde{r}(\{d, 0,4,6\})=$ $\tilde{r}(\{d, 0,4,5\})=\{d, 0,4,5\}$. Similarly, we have $\tilde{r}(\{6-10,0,7,12\})=\{6-10,0,7,11\}$, $\tilde{r}(\{6-10,0,3,7\})=\{6-10,0,1,7\}$, and $\tilde{r}(\{d, 0,7,10\})=\{d, 0,4,5\}$. Consequently, the boundary word is rewritten in terms of critical 1-cells as follows:

$$
\tilde{r}(\partial(\{d, 6-10,0,7\}))=\{6-10,0,7,11\}\{d, 0,4,5\}\{6-10,0,1,7\}^{-1}\{d, 0,4,5\}^{-1} .
$$


Applying the rewriting map, we can obtain the boundary words of other critical 2-cells in terms of critical 1-cells as follows:

$$
\begin{aligned}
\tilde{r}(\partial(\{d, 6-10,4,7\})) & =\{6-10,0,7,11\}\{d, 4,5,6\}\{6-10,0,1,7\}^{-1}\{d, 4,5,6\}^{-1}, \\
\tilde{r}(\partial(\{d, 6-10,7,11\})) & =\{6-10,7,11,12\}\{d, 4,5,6\}\{6-10,0,7,11\}^{-1}\{d, 4,5,6\}^{-1}, \\
\tilde{r}(\partial(\{d, 6-10,7,8\})) & =\{6-10,7,8,11\}\{d, 4,5,6\}\{6-10,0,7,8\}^{-1}\{d, 4,5,6\}^{-1} .
\end{aligned}
$$

We eliminate the generators $\{6-10,0,7,11\},\{6-10,7,11,12\}$ and $\{6-10,7,8,11\}$ by applying a Tietze transformation. Then there are seven generators

$$
\begin{gathered}
\{d, 0,1,2\},\{d, 0,1,4\},\{d, 0,4,5\},\{d, 4,5,6\}, \\
\{6-10,0,1,7\},\{6-10,0,7,8\},\{6-10,7,8,9\}
\end{gathered}
$$

and one relation $\left[\{d, 0,4,5\}^{-1}\{d, 4,5,6\},\{6-10,0,1,7\}\right]$. Also, we can introduce a new generator $g$ by adding the relation $g=\{d, 0,4,5\}^{-1}\{d, 4,5,6\}$ and then eliminate the generator $\{d, 4,5,6\}$ by applying a Tietze transformation. Then we obtain the presentation of $B_{4} S_{0}$ with seven generators $g,\{d, 0,1,2\},\{d, 0,1,4\},\{d, 0,4,5\}$, $\{6-10,0,1,7\},\{6-10,0,7,8\},\{6-10,7,8,9\}$ and one relator $[g,\{6-10,0,1,7\}]$. Thus $B_{4}\left(S_{0}\right)$ is a right-angled Artin group. Note that $B_{n}\left(S_{0}\right)$ is not a right-angled Artin group for $n \geq 5$, as we will see in 4 .

We now explain how to obtain a geometric graph braid corresponding to a critical 1-cell. Given a graph $\Gamma$ ordered by a planar embedding of its maximal tree, the 1-skeleton of the Morse complex is a bouquet consisting of critical 1-cells. So the subcomplex consisting of all 0 -cells and all collapsible 1-cells in $U D_{n} \Gamma$ forms a maximal tree $S$ of the 1-skeleton of $U D_{n} \Gamma$. Let $c=\left\{e, v_{1}, \ldots, v_{n-1}\right\}$ be a critical 1cell in $U D_{n} \Gamma$ and $0_{n}=\{0,1, \ldots, n-1\}$ be the base point of $U D_{n} \Gamma$. Then there are unique edge paths $\alpha$ from $0_{n}$ and $\left\{\iota(e), v_{1}, \ldots, v_{n-1}\right\}$ and $\beta$ from $\left\{\tau(e), v_{1}, \ldots, v_{n-1}\right\}$ and $0_{n}$ in the tree $S$. The edge loop $\alpha e \beta$ is a typical generator of $B_{n} \Gamma$. The edge loop uniquely corresponds to a geometric $n$-braid on $\Gamma$ given by the motion of $n$ vertices in $0_{n}$ along $\alpha e \beta$.

For example, the critical 1-cell $\{6-10,7,8,11\}$ of $U D_{4} S_{0}$ in Example 2.3 determines the edge loop given by

$$
\begin{aligned}
& \{0,1,2,3\} \rightarrow\{0,1,2,4\} \rightarrow\{0,1,2,5\} \rightarrow \cdots \rightarrow\{0,1,2,11\} \rightarrow\{0,1,3,11\} \\
& \rightarrow \cdots \rightarrow\{0,1,10,11\} \rightarrow \cdots \rightarrow\{0,8,10,11\} \rightarrow\{1,8,10,11\} \rightarrow \cdots \rightarrow\{7,8,10,11\} \\
& \rightarrow\{6,7,8,11\} \rightarrow\{5,7,8,11\} \rightarrow \cdots \rightarrow\{0,7,8,11\} \rightarrow\{0,6,8,11\} \\
& \rightarrow \cdots \rightarrow\{0,1,8,11\} \rightarrow\{0,1,7,11\} \rightarrow \cdots \rightarrow\{0,1,2,11\} \rightarrow \cdots \rightarrow\{0,1,2,3\},
\end{aligned}
$$

where the 1-cell $\{6-10,7,8,11\}$ gives the edge path $\{7,8,10,11\} \rightarrow\{6,7,8,11\}$. This loop corresponds to the geometric 4-braid in Figure 4(a).

Similarly the critical 1-cell $\{d, 0,1,2\}(=\{0,1,2,12\} \rightarrow\{0,1,2,3\})$ of $U D_{4} S_{0}$ determines the edge loop given by

$$
\{0,1,2,3\} \rightarrow\{0,1,2,4\} \rightarrow \cdots \rightarrow\{0,1,2,12\} \rightarrow\{0,1,2,3\}
$$

and corresponds to the geometric 4-braid in Figure 4 (b).

Since geometric braids permute the $n$ vertices in $0_{n}$, there is a natural homomorphism $\pi$ from $B_{n} \Gamma$ into the symmetric group $S_{n}$. If the vertex $i$ in $0_{n}$ is regarded as a puncture labeled by $n-i$ for $0 \leq i \leq n-1$, then $\pi(\{6-10,7,8,11\})=(4,3,2)$ and $\pi(\{d, 0,1,2\})$ is the identity. The homomorphism $\pi$ obviously depends on the 


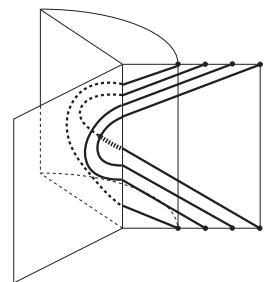

(a) $\{6-10,7,8,11\}$

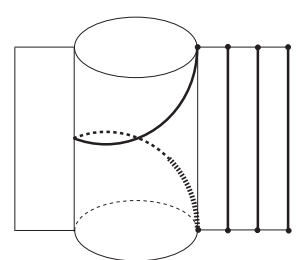

(b) $\{d, 0,1,2\}$

Figure 4. Geometric graph braids on $S_{0}$

choice of labels, but it is well-defined up to conjugation. Thus for a graph $\Gamma$ ordered by a planar embedding of its maximal tree, there is a well-defined homomorphism

$$
\pi_{*}: H_{1}\left(B_{n} \Gamma\right) \rightarrow H_{1}\left(S_{n}\right)=\mathbb{Z}_{2}
$$

which will be useful in 4.4

When we work with an arbitrary graph of an arbitrary braid index $n$, it is convenient to represent cells of $U D_{n}(\Gamma)$ by using the following notation adapted from [9. Let $A$ be a vertex of valency $\mu \geq 3$ in a maximal tree of $\Gamma$. Starting from the branch clockwise next to the branch joining the base vertex, we number branches incident to $A$ clockwise. Let $\vec{a}$ be a vector $\left(a_{1}, \ldots, a_{\mu-1}\right)$ of non-negative integers and let $|\vec{a}|=\sum_{i=1}^{\mu-1} a_{i}$. Then for $1 \leq k \leq \mu-1, A_{k}(\vec{a})$ denotes the set consisting of one edge $e$ with $\tau(e)=A$ that lies on the $k$-th branch together with $a_{i}$ blocked vertices that lie on the $i$-th branch if $i \neq k$ and $a_{k}-1$ blocked vertices on the $k$-th branch. For $1 \leq s \leq n, 0_{s}$ denotes the set $\{0,1, \ldots, s-1\}$ of $s$ consecutive vertices from the base vertex. Let $\dot{A}(\vec{a})$ denote the set of vertices consisting of $A$ together with $a_{i}$ blocked vertices that lie on the $i$-th branch, and let $A(\vec{a})=\dot{A}(\vec{a})-\{A\}$. Then $A(\vec{a})$ can be obtained from $A_{k}(\vec{a})$ by replacing an edge $e$ with $\iota(e)$. Every critical $i$-cell is represented by the following union:

$$
A_{k_{1}}^{1}\left(\vec{a}^{1}\right) \cup \cdots \cup A_{k_{p}}^{p}\left(\vec{a}^{p}\right) \cup\left\{d_{1}, \ldots, d_{q}\right\} \cup\left\{v_{1}, \ldots, v_{r}\right\} \cup 0_{s},
$$

where $A^{1}, \ldots, A^{p}$ are vertices of valency $\geq 3, d_{1}, \ldots, d_{q}$ are deleted edges, and $v_{1}, \ldots, v_{r}$ are blocked vertices blocked by deleted edges. Furthermore, since $s$ is uniquely determined by $s=n-\left(\left|\vec{a}^{1}\right|+\cdots+\left|\vec{a}^{p}\right|+q+r\right)$, we will omit $0_{s}$ in the notation.

We now prove the conjecture by Abrams for braid index $n \geq 3$. Abrams himself proved the conjecture for $n=2$ in [1].

Theorem 2.4. Suppose that $\Gamma$ is a graph with at least $n(\geq 3)$ vertices such that each path joining two vertices of valency $\neq 2$ contains at least $n-1$ edges and each simple loop contains at least $n+1$ edges. Then $U D_{n} \Gamma\left(D_{n} \Gamma\right.$, respectively) is a deformation retract of $U C_{n} \Gamma\left(C_{n} \Gamma\right.$, respectively).

Proof. We first choose a maximal tree $T$ of $\Gamma$ so that one of the end vertices of every deleted edge has valency $\geq 3$ in $\Gamma$, the base vertex 0 has valency 1 in $T$ and the path between 0 and the nearest vertex of valency $\geq 3$ in $T$ contains at least $n-1$ edges. Then we give an order on vertices of $T$ by choosing a planar embedding of $T$. Let $\Gamma^{\prime}$ be obtained by subdividing $\Gamma$ so that each path joining two vertices of valency $\geq 3$ contains at least $n+1$ edges. When we subdivide, we do not touch any deleted 
edge. We note that the assumption $n \geq 3$ is needed here. A maximal tree $T^{\prime}$ of $\Gamma^{\prime}$ and its planar embedding are obtained by subdividing $T$ so that an order on $T^{\prime}$ is assigned accordingly. Since $\Gamma^{\prime}$ is a subdivision of $\Gamma$, vertices of valency $\geq 3$ in $\Gamma$ and in $\Gamma^{\prime}$ coincide and deleted edges in $\Gamma$ and in $\Gamma^{\prime}$ also coincide. We use the same notation to denote two coinciding objects. Let $K_{i}$ (and $K_{i}^{\prime}$, respectively) be the set of all critical $i$-cells in $U D_{n} \Gamma$ (and $U D_{n} \Gamma^{\prime}$ ). Each cell in $K_{i}$ containing non-orderrespecting edges given by $A_{k}(\vec{a})$ 's and deleted edges $d$ 's can be identified with the $i$-cell in $K_{i}^{\prime}$ containing the non-order-respecting edges and the deleted edges that are denoted by the same notation. Thus $K_{i}$ can be naturally regarded as a subset of $K_{i}^{\prime}$ for each $i$. We remark that a non-order-respecting edge in $K_{i}^{\prime}$ determined by $A_{k}(\vec{a})$ may be a subdivided part of the corresponding non-order-respecting edge in $K_{i}$.

Due to our requirement on the base vertex, $K_{0}=K_{0}^{\prime}$ consists of a single critical 0 -cell. Let $\tilde{r}\left(\tilde{r}^{\prime}\right.$, respectively) be the rewriting map of 1-cells in terms of critical 1-cells in $U D_{n} \Gamma$ (and $\left.U D_{n} \Gamma^{\prime}\right)$. The fundamental group $\pi_{1}\left(U D_{n} \Gamma\right)\left(\pi_{1}\left(U D_{n} \Gamma^{\prime}\right)\right.$, respectively) is generated over $K_{1}\left(K_{1}^{\prime}\right.$, respectively) and related by $\tilde{r}(\partial c)$ for each $c \in K_{2}$ ( $K_{2}^{\prime}$, respectively). Our first goal is to obtain an isomorphism between $\pi_{1}\left(U D_{n} \Gamma\right)$ and $\pi_{1}\left(U D_{n} \Gamma^{\prime}\right)$ which can be established by applying Tietze transformations if we show the following two facts:

(1) For each $c \in K_{2}, \tilde{r}(\partial c)=\tilde{r}^{\prime}(\partial c)$.

(2) There is a bijection $h: K_{2}^{\prime}-K_{2} \rightarrow K_{1}^{\prime}-K_{1}$ such that for each $c \in K_{2}^{\prime}-K_{2}$, the word $\tilde{r}^{\prime}(\partial c)$ contains $h(c)$ or $h(c)^{-1}$ exactly once.

For a vertex $v$ in a tree $T$ ordered by its planar embedding, the location of $v$ is completely determined by the triple $\left(Y_{T}(v), \beta_{T}(v), \delta_{T}(v)\right)$, where $Y_{T}(v)$ is the nearest smaller vertex of valency $\geq 3$ or the base vertex 0 if no such vertex exists, $v$ lies on the $\beta_{T}(v)$-th branch of $Y_{T}(v)$ clockwise from the branch to the base vertex, and $\delta_{T}(v)$ is the number of edges in the shortest path from $v$ to $Y_{T}(v)$. If $v$ itself is of valency $\geq 3$ or the base vertex, then $\beta_{T}(v)=\delta_{T}(v)=0$. A 1-cell $b^{\prime}$ in $U D_{n} \Gamma^{\prime}$ with a non-order-respecting edge or a deleted edge is said to be realizable in $\Gamma$ if there is a 1-cell $b$ in $U D_{n} \Gamma$ with the same non-order-respecting edge or the same deleted edge such that for each $v^{\prime} \in b^{\prime},\left(Y_{T^{\prime}}\left(v^{\prime}\right), \beta_{T^{\prime}}\left(v^{\prime}\right), \delta_{T^{\prime}}\left(v^{\prime}\right)\right)=\left(Y_{T}(v), \beta_{T}(v), \delta_{T}(v)\right)$ for some $v \in b$.

For $c \in K_{2}$, we try to compute $\tilde{r}^{\prime}(\partial c)$. Whenever we encounter a redundant 1cell $b^{\prime}$ that is not realizable in $\Gamma$ on the course of this computation, $b^{\prime}$ must contain exactly one vertex $v^{\prime}$ such that $\delta_{T^{\prime}}\left(v^{\prime}\right)>\delta_{T}(v)$ for any vertex $v$ of $T$ satisfying $Y_{T}(v)=Y_{T^{\prime}}\left(v^{\prime}\right)$ and $\beta_{T}(v)=\beta_{T^{\prime}}\left(v^{\prime}\right)$. We move $v^{\prime}$ forward by applying $V_{e}$ in Lemma 2.2 as many times as needed until $b^{\prime}$ becomes realizable in $\Gamma$. It is now clear that $\tilde{r}$ and $\tilde{r}^{\prime}$ yield the same output. This proves part (1).

To show part (2), we first characterize critical cells in $K_{2}^{\prime}-K_{2}$ and $K_{1}^{\prime}-K_{1}$. A pair of vertices $A, B$ of valency $\geq 3$ in $\Gamma$ are said to be restrictive if the shortest path joining $A$ and $B$ in $\Gamma$ contains exactly $n-1$ edges, every vertex on the interior of the path has valency 2 in $\Gamma$, and there is a deleted edge $d$ on the path such that its one end vertex is $B$. In order to have $K_{2}^{\prime}-K_{2} \neq \emptyset$ and $K_{1}^{\prime}-K_{1} \neq \emptyset$, it is necessary that there is at least one restrictive pair in $\Gamma$. Let $\mu$ be the valency of $A$ in $T$ and assume that $d$ is on the $k_{0}$-th branch at $A$ for some $1 \leq k_{0} \leq \mu$. Let $\vec{\delta}_{i}$ be the $\mu$-dimensional unit vector whose $i$-th component equals 1 . 


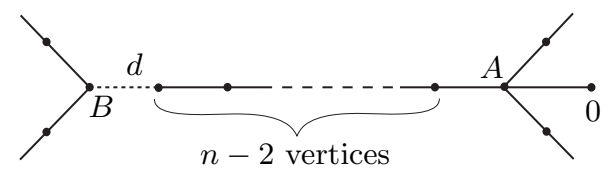

(a) Restrictive pair

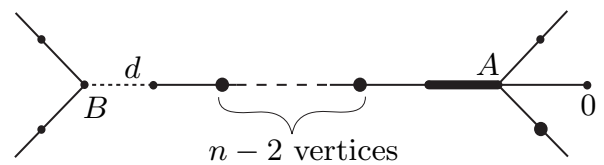

(c) $A_{k_{0}}\left((n-1) \vec{\delta}_{k_{0}}+\vec{\delta}_{k}\right)$

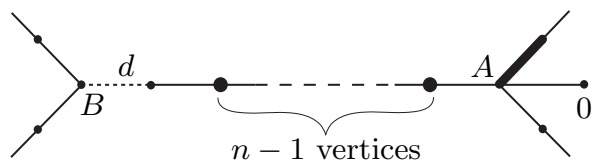

(b) $A_{k}\left((n-1) \vec{\delta}_{k_{0}}+\vec{\delta}_{k}\right)$

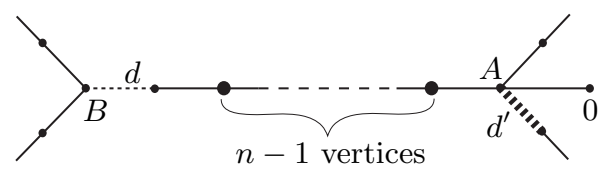

(d) $d^{\prime} \cup A\left((n-1) \vec{\delta}_{k_{0}}\right)$

FiguRe 5. Critical 1-cells in $K_{1}^{\prime}-K_{1}$

There are three kinds of critical 2-cells in $K_{2}^{\prime}-K_{2}$ that can be described by using the notation of a restrictive pair (see Figure 5):

(i) $d \cup A_{k}\left((n-2) \vec{\delta}_{k_{0}}+\vec{\delta}_{k}\right)$ for $k_{0}<k \leq \mu$;

(ii) $d \cup d^{\prime} \cup A\left((n-2) \vec{\delta}_{k_{0}}\right)$ for a deleted edge $d^{\prime}$ with an end vertex $A$;

(iii) $d \cup A_{k_{0}}\left((n-2) \vec{\delta}_{k_{0}}+\vec{\delta}_{k}\right)$ for some $1 \leq k<k_{0}$.

There are also three corresponding kinds of critical 1-cells in $K_{1}^{\prime}-K_{1}$ :

(i) $A_{k}\left((n-1) \vec{\delta}_{k_{0}}+\vec{\delta}_{k}\right)$ for $k_{0}<k \leq \mu$;

(ii) $d^{\prime} \cup A\left((n-1) \vec{\delta}_{k_{0}}\right)$ for a deleted edge $d^{\prime}$ with an end vertex $A$;

(iii) $A_{k_{0}}\left((n-1) \vec{\delta}_{k_{0}}+\vec{\delta}_{k}\right)$ for some $1 \leq k<k_{0}$.

We define a bijection $h: K_{2}^{\prime}-K_{2} \rightarrow K_{1}^{\prime}-K_{1}$ by sending a critical 2-cell in $K_{2}^{\prime}-K_{2}$ to the corresponding critical 1-cell in $K_{1}^{\prime}-K_{1}$. It is not hard to see that the word $\tilde{r}^{\prime}(\partial c)$ contains $h(c)$ or $h(c)^{-1}$ exactly once. For example, $\partial\left(d \cup A_{k}\left((n-2) \vec{\delta}_{k_{0}}+\vec{\delta}_{k}\right)\right)$ contains $\left\{v_{d}\right\} \cup A_{k}\left((n-2) \vec{\delta}_{k_{0}}+\vec{\delta}_{k}\right)$ or its inverse exactly once, where $v_{d}$ is the end vertex of $d$ that is not $B$. But

$$
\tilde{r}^{\prime}\left(\left\{v_{d}\right\} \cup A_{k}\left((n-2) \vec{\delta}_{k_{0}}+\vec{\delta}_{k}\right)\right)=\tilde{r}^{\prime}\left(A_{k}\left((n-1) \vec{\delta}_{k_{0}}+\vec{\delta}_{k}\right)\right)
$$

by Lemma 2.2 ,

In order to realize this isomorphism of fundamental groups by a continuous map, we need to consider an intermediate cubical complex $\overline{U D}_{n}$ obtained by subdividing $U D_{n} \Gamma$ as follows: Whenever an edge $e$ in $\Gamma$ is subdivided into $e_{1}, \ldots, e_{\ell}$ in $\Gamma^{\prime}$, we replace each cell $\left\{e, x_{1} \ldots x_{i}\right\}$ containing $e$ by $(2 \ell-1)$ cells $\left\{e_{1}, x_{1} \ldots x_{i}\right\}$, $\left\{v_{1}, x_{1} \ldots x_{i}\right\},\left\{e_{2}, x_{1} \ldots x_{i}\right\}, \ldots,\left\{v_{\ell-1}, x_{1} \ldots x_{i}\right\},\left\{e_{\ell}, x_{1} \ldots x_{i}\right\}$, where $v_{j}=\tau\left(e_{j}\right)$ $=\iota\left(e_{j+1}\right)$. Then there is an obvious cubical embedding $\bar{i}: \overline{U D}_{n} \hookrightarrow U D_{n} \Gamma^{\prime}$. We remark that $U D_{n} \Gamma^{\prime}$ have more cells, such as one containing more than one vertice or edge produced by a subdivision of an edge. The cubical complex $\overline{U D}_{n}$ still has the obvious 0-cell as the basepoint. A critical cell $c$ in $U D_{n} \Gamma$ may become a union $\sigma$ of cells $\overline{U D}_{n}$. Except for the critical cell corresponding to $c$ in $U D_{n} \Gamma^{\prime}$, all other cells in $\bar{i}(\sigma)$ inevitably contain an order-respecting edge preceded by an unblocked vertex, and so they are redundant in $U D_{n} \Gamma^{\prime}$. Thus the isomorphism $: \pi_{1}\left(U D_{n} \Gamma\right) \rightarrow \pi_{1}\left(U D_{n} \Gamma^{\prime}\right)$ established above is induced from the composite of the identity map : $U D_{n} \Gamma \rightarrow \overline{U D}_{n}$ and the inclusion $\bar{i}: \overline{U D}_{n} \rightarrow U D_{n} \Gamma^{\prime}$. 
Since both $\overline{U D}_{n}$ and $U D_{n} \Gamma^{\prime}$ are $K(\pi, 1)$-spaces, $\bar{i}$ is a homotopy equivalence. As Abrams showed in [1, $U D_{n} \Gamma^{\prime}$ is a deformation retract of $U C_{n} \Gamma$. Thus the composition of two inclusions $\bar{i}: \overline{U D}_{n} \hookrightarrow U D_{n} \Gamma^{\prime}$ and $U D_{n} \Gamma^{\prime} \hookrightarrow U C_{n} \Gamma$ is a homotopy equivalence. Since involved cubical complexes are all finite, this composition has the homotopy extension property, and so $\overline{U D}_{n}$ is a deformation retract of $U C_{n} \Gamma$. Since $\overline{U D}_{n}$ and $U D_{n} \Gamma$ share the same underlying space, we complete a proof for the unordered case.

Recall the covering $D_{n} \Gamma \rightarrow U C_{n} \Gamma=D_{n} \Gamma / S_{n}$ for the symmetric group $S_{n}$. Let $\bar{p}: \bar{D}_{n} \rightarrow \overline{U D}_{n}$ be the covering with the deck transformation group $S_{n}$. It is clear that the cover $\bar{D}_{n}$ can be obtained by subdividing $D_{n} \Gamma$ in a similar way to subdividing $U D_{n} \Gamma$ in order to obtain $\overline{U D}_{n}$ so that there is a cubical embedding $\tilde{i}: \bar{D}_{n} \rightarrow D_{n} \Gamma^{\prime}$. Then we naturally have $\bar{i} \bar{p}=p^{\prime} \tilde{i}$, where $p^{\prime}: D_{n} \Gamma^{\prime} \rightarrow U D_{n} \Gamma^{\prime}$ is the $S_{n}$-covering. Then $\tilde{i}_{*}: \pi_{1}\left(\bar{D}_{n}\right) \rightarrow \pi_{1}\left(D_{n} \Gamma^{\prime}\right)$ is injective since $\bar{i}_{*}$ is an isomorphism. Considering indices of subgroups, we have

$$
\left[\pi_{1}\left(U D_{n} \Gamma\right): \operatorname{im} p_{*}^{\prime}\right]\left[\pi_{1}\left(D_{n} \Gamma^{\prime}\right): \operatorname{im} \tilde{i}_{*}\right]=\left[\pi_{1}\left(U D_{n} \Gamma^{\prime}\right): \operatorname{im} \bar{i}_{*}\right]\left[\pi_{1}\left(\overline{U D}_{n}\right): \operatorname{im} \bar{p}_{*}\right],
$$

and so $\left[\pi_{1}\left(D_{n} \Gamma^{\prime}\right): \operatorname{im} \tilde{i}_{*}\right]=1$. Thus $\tilde{i}_{*}$ is an isomorphism. Since both $\bar{D}_{n}$ and $D_{n} \Gamma^{\prime}$ are $K(\pi, 1)$-spaces, $\tilde{i}$ is a homotopy equivalence. By a similar argument to the unordered case, we obtain the ordered case.

It is clear from the above proof that the existence of restrictive pairs is not only necessary but also sufficient to guarantee $K_{2}^{\prime}-K_{2} \neq \emptyset$ and $K_{1}^{\prime}-K_{1} \neq \emptyset$, perhaps except for the case $n=2$. It is also clear that if each path in $\Gamma$ joining two vertices of valency $\geq 3$ contains at least $n$ edges, then $K_{2}^{\prime}-K_{2}=K_{1}^{\prime}-K_{1}=\emptyset$.

2.3. Proof of Theorem A. We say that a graph $\Gamma$ contains another graph $\Gamma^{\prime}$ if a subdivision of $\Gamma^{\prime}$ is a subgraph of a subdivision of $\Gamma$.

Theorem A. If a graph $\Gamma$ does not contain $T_{0}$ and $S_{0}$ in Figure 1 , then $B_{n}(\Gamma)$ is a right-angled Artin group for any braid index.

Proof. Suppose that a graph $\Gamma$ contains neither $T_{0}$ nor $S_{0}$. Since $\Gamma$ does not contain $T_{0}$, a maximal tree of $\Gamma$ must be a linear tree, that is, the tree contains a simple path containing all vertices of valency $\geq 3$. Moreover, since $\Gamma$ does not contain $S_{0}$, every circuit cannot contain more than one vertex of valency $\geq 3$, and so circuits form bouquets. Thus $\Gamma$ must look like a graph in Figure 6 that we call a linear star-bouquet.

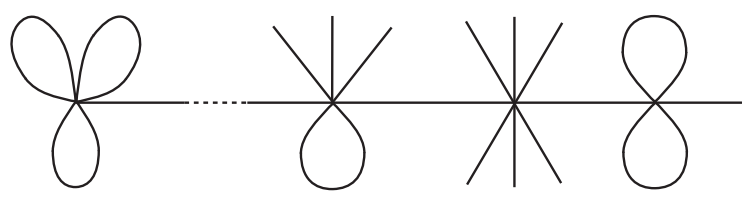

Figure 6. Graph $\Gamma$ with no $T_{0}$ or $S_{0}$

If we choose a maximal tree of $\Gamma$ and give an order as in Figure 7 , the complex $U D_{n} \Gamma$ has two kinds of critical 1-cells:

$$
d_{p}^{i} \cup A^{i}(\vec{a}), \quad A_{k}^{i}(\vec{a})
$$

and four kinds of critical 2-cells: For $i<j$

$A_{k}^{i}(\vec{a}) \cup A_{\ell}^{j}(\vec{b}), \quad d_{p}^{i} \cup d_{q}^{j} \cup A^{i}(\vec{a}) \cup A^{j}(\vec{b}), \quad A_{k}^{i}(\vec{a}) \cup d_{q}^{j} \cup A^{j}(\vec{b}), \quad d_{p}^{i} \cup A_{\ell}^{j}(\vec{b}) \cup A^{i}(\vec{a})$. 
Here $A^{i}$ is the $i$-th vertex of valency $\geq 3$ in the maximal tree and $d_{j}^{i}$ is the $j$-th of deleted edges that ends at the vertex $A^{i}$. See Figure 7.

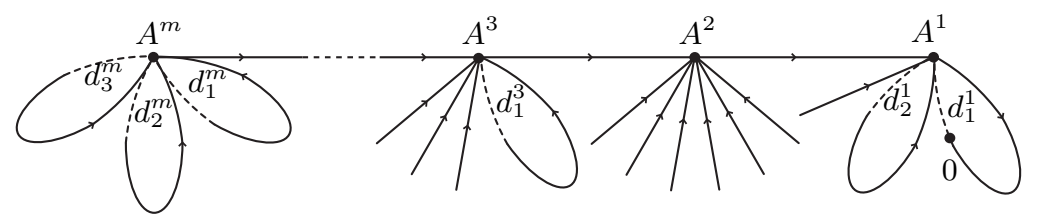

Figure 7. Maximal tree of $\Gamma$

Relations of $B_{n} \Gamma$ are obtained by rewriting boundary words of critical 2-cells as words in critical 1-cells. Let $\mu$ be the valency of $A^{i}$ in the maximal tree. The boundary word of the critical 2-cell $A_{k}^{i}(\vec{a}) \cup A_{\ell}^{j}(\vec{b})$ is given by the product of 1cells $\left(A_{k}^{i}(\vec{a}) \cup A^{j}(\vec{b})\right)\left(\left(\dot{A}^{i}(\vec{a})-A^{i}\left(\vec{\delta}_{k}\right)\right) \cup A_{\ell}^{j}(\vec{b})\right)\left(A_{k}^{i}(\vec{a}) \cup\left(\dot{A}^{j}(\vec{b})-A^{j}\left(\vec{\delta}_{\ell}\right)\right)\right)^{-1}\left(A^{i}(\vec{a}) \cup\right.$ $\left.A_{\ell}^{j}(\vec{b})\right)^{-1}$, where $\vec{\delta}_{k}$ denotes the $k$-th coordinate unit vector. Since

$$
\begin{gathered}
\tilde{r}\left(A^{i}(\vec{a}) \cup A_{\ell}^{j}(\vec{b})\right)=\tilde{r}\left(\left(\dot{A}^{i}(\vec{a})-A^{i}\left(\vec{\delta}_{k}\right)\right) \cup A_{\ell}^{j}(\vec{b})\right)=A_{\ell}^{j}(\vec{b}) \\
\text { and } \tilde{r}\left(A_{k}^{i}(\vec{a}) \cup A^{j}(\vec{b})\right)=\tilde{r}\left(A_{k}^{i}(\vec{a}) \cup\left(\dot{A}^{j}(\vec{b})-A^{j}\left(\vec{\delta}_{\ell}\right)\right)\right)=A_{k}^{i}\left(\vec{a}+|\vec{b}| \vec{\delta}_{\mu-1}\right),
\end{gathered}
$$

the relation becomes

$$
\left[A_{k}^{i}\left(\vec{a}+|\vec{b}| \vec{\delta}_{\mu-1}\right), A_{\ell}^{j}(\vec{b})\right],
$$

which is a commutator of critical 1-cells. Similarly, we can obtain relations from the boundary words of the remaining three types of critical 2-cells. Then they are all commutators of critical 1-cells as follows:

$$
\begin{aligned}
& {\left[d_{p}^{i} \cup A^{i}\left(\vec{a}+(|\vec{b}|+1) \vec{\delta}_{\mu-1}\right), d_{q}^{j} \cup A^{j}(\vec{b})\right],} \\
& {\left[A_{k}^{i}\left(\vec{a}+(|\vec{b}|+1) \vec{\delta}_{\mu-1}\right), d_{q}^{j} \cup A^{j}(\vec{b})\right], \quad\left[d_{p}^{i} \cup A^{i}\left(\vec{a}+|\vec{b}| \vec{\delta}_{\mu-1}\right), A_{\ell}^{j}(\vec{b})\right] .}
\end{aligned}
$$

Consequently, $B_{n} \Gamma$ is a right-angled Artin group.

\section{Graphs Containing $T_{0}$ BUt not $S_{0}$}

Let $K$ be a finite simplicial complex. Then the exterior face algebra $\Lambda[K]$ of $K$ over a given field is defined by

$$
\Lambda[K]=\Lambda\left[v_{1}, \ldots, v_{m}\right] / I,
$$

where $\Lambda\left[v_{1}, \ldots, v_{m}\right]$ is the exterior algebra generated by vertices of $K$ over a field and $I$ is the ideal generated by the products $v_{i_{1}} \cdots v_{i_{\ell}}$ such that $\left\{v_{i_{1}}, \ldots, v_{i_{\ell}}\right\}$ does not form a simplex in $K$. The field $\mathbb{Z}_{2}$ will be used in this article. In this case, the algebra $\Lambda[K]$ can be regarded as a Stanley-Reisner ring, and it was shown by Bruns and Gubeladze in [3] that $\Lambda[K]$ completely determines a finite simplicial complex $K$. That is, if $\Lambda[K]$ and $\Lambda\left[K^{\prime}\right]$ are isomorphic for finite simplicial complexes $K$ and $K^{\prime}$, then there is a simplicial homeomorphism between $K$ and $K^{\prime}$.

A simplicial complex $K$ is called a flag complex if every complete subgraph in a 1-skeleton spans a simplex in $K$. The following proposition provides our guiding principle in this section. 
Proposition 3.1. (1) (Charney-Davis [5]) Let $G$ be a right-angled Artin group. The Eilenberg-MacLane space $K(G, 1)$ is a full subcomplex of a high-fold torus. In particular, the cohomology algebra $H^{*}\left(G ; \mathbb{Z}_{2}\right)$ is an exterior face algebra of a flag complex.

(2) (Farley-Sabalka 10]) Let $K$ and $K^{\prime}$ be finite simplicial complexes. If $\phi$ : $\Lambda(K) \rightarrow \Lambda\left(K^{\prime}\right)$ is a degree-preserving surjection, $K$ is a flag complex and $\operatorname{ker}(\phi)$ is generated by homogeneous elements of degree 1 and 2 , then $K^{\prime}$ is also a flag complex.

Let $\Gamma$ and $\Gamma^{\prime}$ be graphs such that $\Gamma$ contains $\Gamma^{\prime}$. If homology groups $H_{k}\left(B_{n} \Gamma\right)$ and $H_{k}\left(B_{n^{\prime}} \Gamma^{\prime}\right)$ are torsion-free for $k \geq 0$ and $n^{\prime} \leq n$ and there exists an embedding $i: \Gamma^{\prime} \rightarrow \Gamma$ which induces an injection $i_{*}: H_{k}\left(B_{n^{\prime}} \Gamma^{\prime}\right) \rightarrow H_{k}\left(B_{n} \Gamma\right)$ sending generators to generators, then the induced map $i^{*}: H^{*}\left(B_{n} \Gamma ; \mathbb{Z}_{2}\right) \rightarrow H^{*}\left(B_{n^{\prime}} \Gamma^{\prime} ; \mathbb{Z}_{2}\right)$ is surjective by the universal coefficient theorem. Also, if $H^{*}\left(B_{n^{\prime}} \Gamma^{\prime} ; \mathbb{Z}_{2}\right)$ is an exterior face algebra of a non-flag complex, the induced map $i^{*}$ is degree-preserving and $\operatorname{ker}\left(i^{*}\right)$ is generated by homogeneous elements of degree 1 and 2 , then $H^{*}\left(B_{n} \Gamma ; \mathbb{Z}_{2}\right)$ is not an exterior face algebra of a flag complex by Proposition $3.1(2)$. So $B_{n} \Gamma$ is not a right-angled Artin group by Proposition 3.1(1). In the case where $\Gamma^{\prime}=T_{0}$ and $\Gamma$ is a tree $T$ containing $T_{0}$, Farley and Sabalka employed this argument in [10] to prove that $B_{n} T$ is not a right-angled Artin group for $n \geq 4$. We try to do the same for the case where $\Gamma^{\prime}=T_{0}$ and $\Gamma$ is a graph containing $T_{0}$. There are several difficulties, as we now explain.

If a graph contains $S_{0}$ as well as $T_{0}$, such as the graph $T^{\prime}$ given in Figure 8 , then one of the hypotheses of the above argument fails to hold. In fact, one can show that there is no embedding $i: T_{0} \rightarrow T^{\prime}$ which induces an injection $i_{*}$ : $H_{1}\left(B_{4} T_{0}\right) \rightarrow H_{1}\left(B_{n} T^{\prime}\right)$ for $n \geq 4$. For a graph $\Gamma$ containing $S_{0}$, we introduce some other argument in $\$ 4$ to prove that $B_{n} \Gamma$ is not a right-angled Artin group.

Let $\mathcal{G}$ be the set consisting of graphs that contain $T_{0}$ but do not contain $S_{0}$. Let $T_{1}, T_{2}$ and $T_{3}$ be graphs depicted in Figure 8. Then they are graphs in $\mathcal{G}$. One can also show that for each $k=0,1,2$, there is no embedding $T_{k} \rightarrow T_{k+1}$ which induces an injection $H_{1}\left(B_{4} T_{k}\right) \rightarrow H_{1}\left(B_{n} T_{k+1}\right)$ for $n \geq 4$. In order to apply the above argument, we further need to divide $\mathcal{G}$ into four subclasses: For each $k=0,1,2, \mathcal{G}_{k}$ consists of graphs in $\mathcal{G}$ that contain $T_{k}$ but do not contain $T_{k+1}$ and $\mathcal{G}_{3}$ consists of graphs containing $T_{3}$ but not containing $S_{0}$.

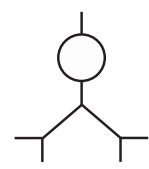

$T^{\prime}$

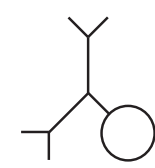

$T_{1}$

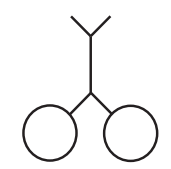

$T_{2}$

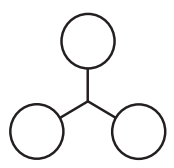

$T_{3}$

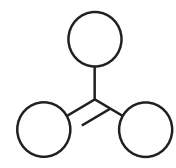

$T_{3}^{\prime}$

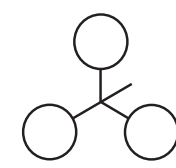

$T_{3}^{\prime \prime}$

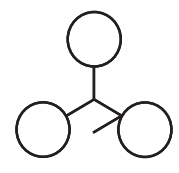

$T_{3}^{\prime \prime \prime}$

Figure 8 
However, we still have one technical problem. The graph $T_{3}$ itself is in $\mathcal{G}_{3}$. In order to show $B_{n} T_{3}$ is not a right-Angled Artin group for $n \geq 2$, we need to find a map $h: T_{3} \rightarrow T_{3}$ that induces an injection $h_{*}: H_{*}\left(U D_{2} T_{3}\right) \rightarrow H_{*}\left(U D_{n} T_{3}\right)$ for $n>2$. The injectivity of $h_{*}$ on homologies forces $h$ to be surjective due to the absence of vertices of valency 1 in $T_{3}$. Then it is impossible for a surjection $h$ to induce a cubical map $U D_{2} T_{3} \rightarrow U D_{n} T_{3}$ for $n>2$, since there is no room to place extra $n-2$ vertices. In $\$ 3.4$, we will show $B_{n} T_{3}$ is not a right-angled Artin group for $n \geq 2$ by using some other method. Also, the subclass $\mathcal{G}_{3}$ is modified so that it consists of graphs that contain $T_{3}^{\prime}, T_{3}^{\prime \prime}$, or $T_{3}^{\prime \prime \prime}$ as depicted in Figure 8 but do not contain $S_{0}$. Then $\mathcal{G}=\mathcal{G}_{0} \cup \mathcal{G}_{1} \cup \mathcal{G}_{2} \cup \mathcal{G}_{3} \cup\left\{T_{3}\right\}$. The goal of this section is to prove the following:

Theorem 3.2. Let $\Gamma$ be a graph in $\mathcal{G}$, that is, it contains $T_{0}$ and does not contain $S_{0}$. Then $B_{n} \Gamma$ is not a right-angled Artin group for $n \geq 4$.

Except for $T_{3}$, this theorem will be proved by the argument mentioned above. In 3.1. we prove that for $\Gamma \in \mathcal{G}$, the homology groups of the Morse complex of $U D_{n} \Gamma$ are free abelian groups generated by critical $k$-cells. In $\$ 3.2$, we prove that the cohomology algebras of $U D_{4} T_{1}, U D_{3} T_{2}, U D_{2} T_{3}^{\prime}, U D_{2} T_{3}^{\prime \prime}$, and $U D_{2} T_{3}^{\prime \prime \prime}$ are exterior face algebras of non-flag complexes. In $\$ 3.3$ we prove that for $\Gamma \in \mathcal{G}_{k}$ for some $k=0,1,2,3$, there is an embedding $i: P_{k} \rightarrow \Gamma$ which induces a surjection $i^{*}$ on cohomology algebras such that $i^{*}$ is degree-preserving and $\operatorname{ker}\left(i^{*}\right)$ is generated by homogeneous elements of degree 1 and 2 , where $P_{k}=T_{k}$ for $k=0,1,2$ and $P_{3}$ is one of $T_{3}^{\prime}, T_{3}^{\prime \prime}$, and $T_{3}^{\prime \prime \prime}$.

3.1. Homologies of $U D_{n} \Gamma$. Let $\Gamma$ be a graph in $\mathcal{G}$. Since $\Gamma$ does not contain $S_{0}$, every circuit cannot contain more than one vertex of valency $\geq 3$, and so circuits form bouquets. Moreover, since $\Gamma$ contains $T_{0}$ but not $S_{0}$, the maximal tree of $\Gamma$ is a non-linear tree. Thus $\Gamma$ looks like a graph in Figure 9(a).

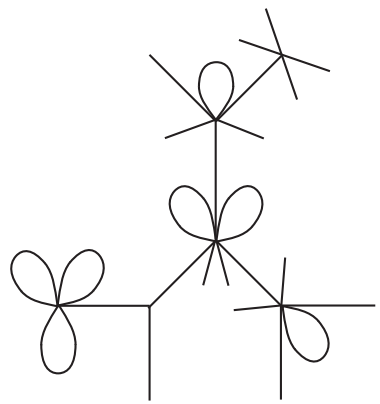

(a) Graph containing $T_{0}$ but not $S_{0}$

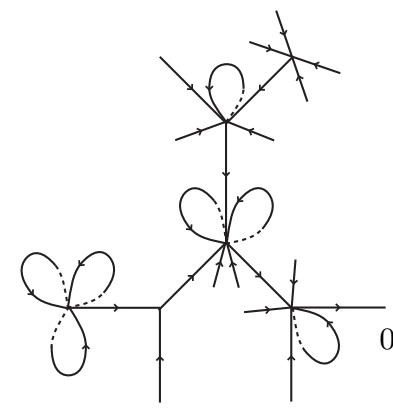

(b) Maximal tree and order

Figure 9. Graph containing $T_{0}$ but not $S_{0}$

Given a graph $\Gamma$, we want to compute the homology of the braid group $B_{n} \Gamma$, which is the same as the homology of $U D_{n} \Gamma$, so we may use its Morse complex instead. Let $\left(C_{i}\left(U D_{n} \Gamma\right), \partial\right)$ be the (cubical) cellular chain complex of $U D_{n} \Gamma$. A choice of a maximal tree and an order on it determine a Morse complex, as described in $\left\{2.2\right.$. Let $M_{i}\left(U D_{n} \Gamma\right)$ be the free abelian group generated by critical $i$-cells. Let 
$c=\left\{e_{1}, e_{2}, \ldots, e_{i}, v_{i+1}, \ldots, v_{n}\right\}$ be an $i$-cell of $U D_{n} \Gamma$, where $e_{1}, \ldots, e_{i}$ are edges with $\iota\left(e_{1}\right)<\iota\left(e_{2}\right)<\cdots<\iota\left(e_{i}\right)$ and $v_{i+1}, \ldots, v_{n}$ are vertices of $\Gamma$ :

$$
\partial(c)=\sum_{k=1}^{i}(-1)^{k}\left(\partial_{k}^{\iota}(c)-\partial_{k}^{\tau}(c)\right)
$$

where

$$
\begin{aligned}
\partial_{k}^{\iota}(c) & =\left\{e_{1}, \ldots, e_{k-1}, e_{k+1}, \ldots, e_{i}, v_{i+1}, \ldots, v_{n}, \iota\left(e_{k}\right)\right\}, \\
\partial_{k}^{\tau}(c) & =\left\{e_{1}, \ldots, e_{k-1}, e_{k+1}, \ldots, e_{i}, v_{i+1}, \ldots, v_{n}, \tau\left(e_{k}\right)\right\} .
\end{aligned}
$$

Consider an abelianized version of the rewriting map $\tilde{r}$ in $\$ 2.2$. Let $R: C_{i}\left(U D_{n} \Gamma\right)$ $\rightarrow C_{i}\left(U D_{n} \Gamma\right)$ be a homomorphism defined by $R(c)=0$ if $c$ is a collapsible $i$-cell, by $R(c)=c$ if $c$ is critical, and by $R(c)= \pm \partial W(c)+c$ if $c$ is redundant where the sign is chosen so that the coefficient of $c$ in $\partial W(c)$ is -1 . By [11, there is a non-negative integer $m$ such that $R^{m}=R^{m+1}$, and let $\widetilde{R}=R^{m}$. Then $\widetilde{R}(c)$ is in $M_{i}\left(U D_{n} \Gamma\right)$ and we have a homomorphism $\widetilde{R}: C_{i}\left(U D_{n} \Gamma\right) \rightarrow M_{i}\left(U D_{n} \Gamma\right)$. This definition is not very different from the map defined by Forman in 11]. But it is easy to see that the two maps are essentially the same. Define a map $\widetilde{\partial}: M_{i}\left(U D_{n} \Gamma\right) \rightarrow M_{i-1}\left(U D_{n} \Gamma\right)$ by $\widetilde{\partial}(c)=\widetilde{R} \partial(c)$. Then $\left(M_{i}\left(U D_{n} \Gamma\right), \widetilde{\partial}\right)$ forms a chain complex. However, the inclusion $M_{*}\left(U D_{n} \Gamma\right) \hookrightarrow C_{*}\left(U D_{n} \Gamma\right)$ is not a chain map. Instead, consider a homomorphism $\varepsilon: M_{i}\left(U D_{n} \Gamma\right) \rightarrow C_{i}\left(U D_{n} \Gamma\right)$ defined as follows: For a (critical) $i$-cell $c, \varepsilon(c)$ is obtained from $c$ by minimally adding collapsible $i$-cells until it becomes closed in the sense that for each redundant $(i-1)$-cell $c^{\prime}$ in the boundary of every $i$-cell summand in $\varepsilon(c), W\left(c^{\prime}\right)$ already appears in $\varepsilon(c)$. Then $\varepsilon$ is a chain map that is a chain homotopy inverse of $\widetilde{R}$. Thus $\left(M_{i}\left(U D_{n} \Gamma\right), \widetilde{\partial}\right)$ and $\left(C_{i}\left(U D_{n} \Gamma\right), \partial\right)$ have the same chain homotopy type, and so their homology groups are isomorphic.

It should be more efficient to compute the homology groups of $U D_{n} \Gamma$ by using its Morse complex if we appropriately choose a maximal tree and an order on $\Gamma$ in $\mathcal{G}$. The following lemma that generalizes the corresponding Farley's result in [8] is useful in the sense that it significantly reduces the amount of computation to evaluate the rewriting map $\widetilde{R}$.

Lemma 3.3. Let $\Gamma$ be a graph. If a cell $c$ in $U D_{n} \Gamma$ contains an order-respecting edge e satisfying

(1) every vertex $v$ in $c$ with $\tau(e)<v<\iota(e)$ is blocked,

(2) for any edge $e^{\prime}$ in $c$ with $\tau(e)<\iota\left(e^{\prime}\right)<\iota(e), \tau(e)<\tau\left(e^{\prime}\right)<\iota(e)$, then $\widetilde{R}(c)=0$.

Proof. The cell $c$ can be either collapsible or redundant. If $c$ is collapsible, then $R(c)=0$ and we are done. So we assume that $c$ is redundant. Then $c$ has the smallest unblocked vertex $v_{s}$. Let $e_{s}$ be the edge in $\Gamma$ such that $\iota\left(e_{s}\right)=v_{s}$. We use induction on the minimal integer $k$ such that $R^{k}(c)=R^{k+1}(c)$. Since $c$ is redundant, $k \geq 2$. Suppose that $k=2$. Then each cell $c^{\prime}$ occurring in $R(c)= \pm \partial W(c)+c$ contains at least one of $e$ and $e_{s}$. Conditions (1) and (2) guarantee that $e$ remains order-respecting. If $c^{\prime}$ does not contain $e, c^{\prime}$ contains $e_{s}$, and $e_{s}$ must be orderrespecting in this case by the choice of $v_{s}$. Since $k=1, c^{\prime}$ can no longer be redundant. Thus $c^{\prime}$ is collapsible, and so $R^{2}(c)=0$.

Suppose that $k \geq 3$. Each cell $c^{\prime}$ occurring in $R(c)$ also contains at least one of $e$ and $e_{s}$. Conditions (1) and (2) guarantee that $e$ remains order-respecting and 
satisfies (1) and (2). If $c^{\prime}$ contains $e_{s}$ and every vertex $v$ in $c^{\prime}$ with $\tau\left(e_{s}\right)<v<\iota\left(e_{s}\right)$ is blocked, then $c^{\prime}$ is collapsible. If $c^{\prime}$ contains $e_{s}$ and there is an unblocked vertex $v$ in $c^{\prime}$ with $\tau\left(e_{s}\right)<v<\iota\left(e_{s}\right)$, then the unblocked vertex is newly formed and $c^{\prime}$ contains $e$ that is order-respecting and still satisfies conditions (1) and (2) because no edges lying between $\tau\left(e_{s}\right)$ and $\iota\left(e_{s}\right)$ are touched. Obviously, $c^{\prime}$ stabilizes at least one application faster than $c$ does under iteration of $R$. By induction, $\widetilde{R}\left(c^{\prime}\right)=0$. If $c^{\prime}$ does not contain $e_{s}$, then $c^{\prime}$ contains the order-respecting edge $e$ that satisfies conditions (1) and (2) because no edges lying between $\tau\left(e_{s}\right)$ and $\iota\left(e_{s}\right)$ are untouched. This also implies $\widetilde{R}\left(c^{\prime}\right)=0$ by induction. Since $\widetilde{R}\left(c^{\prime}\right)=0$ for each cell $c^{\prime}$ occurring in $R(c)$, we have $\widetilde{R}(c)=0$.

For a graph $\Gamma$, let $c=\left\{c_{1}, \ldots, c_{n-1}, v_{s}\right\}$ be an $i$-cell in $U D_{n} \Gamma$. Define a function $V: K_{i} \rightarrow K_{i}$ by $V(c)=\left\{c_{1}, \ldots, c_{n-1}, \tau\left(e_{s}\right)\right\}$ if $c$ is redundant, and $v_{s}$ is the smallest unblocked vertex in $c$, and $e_{s}$ is the edge of $\Gamma$ such that $\iota\left(e_{s}\right)=v_{s}$, and by $V(c)=c$ otherwise. The function $V$ should stabilize to a function $\widetilde{V}: K_{i} \rightarrow K_{i}$ under iteration, that is, $\widetilde{V}=V^{k}$ for some non-negative integer $k$ such that $V^{k}=V^{k+1}$.

Lemma 3.4. Let $\Gamma$ be a graph not containing $S_{0}$. Suppose that we choose a maximal tree of $\Gamma$ and give an order as in Figure 9(b). Let $c$ be a cell in $U D_{n} \Gamma$ given by $c=$ $\left\{c_{1}, \ldots, c_{n-1}, e\right\}$, where $e$ is an edge and each $c_{i}$ are either a vertex or an edge. Then $\widetilde{R} \widetilde{V}\left(c^{\iota}\right)=\widetilde{R} \widetilde{V}\left(c^{\tau}\right)$, where $c^{\iota}=\left\{c_{1}, \ldots, c_{n-1}, \iota(e)\right\}$ and $c^{\tau}=\left\{c_{1}, \ldots, c_{n-1}, \tau(e)\right\}$.

Proof. If $e$ is not a deleted edge or if $e$ is a deleted edge and there are no edges between $\iota(e)$ and $\tau(e)$, then $\iota(e)$ eventually becomes the smallest unblocked vertex and moves via the original location of $\tau(e)$ under iteration of $V$, and so $\widetilde{V}\left(c^{\iota}\right)=$ $\widetilde{V}\left(c^{\tau}\right)$.

If $e$ is a deleted edge and there is an edge $e^{\prime}$ between $\iota(e)$ and $\tau(e)$, then due to our choice of a maximal tree and an order, $e^{\prime}$ is an order-respecting edge satisfying the hypothesis of Lemma 3.3, and so $\widetilde{R} \widetilde{V}\left(c^{\iota}\right)=\widetilde{R} \widetilde{V}\left(c^{\tau}\right)=0$

Farley proved in 8 that $\widetilde{\partial}: M_{i}\left(U D_{n} \Gamma\right) \rightarrow M_{i-1}\left(U D_{n} \Gamma\right)$ is zero for any tree $\Gamma$. We now try to extend this result to graphs containing $T_{0}$ but not $S_{0}$.

Lemma 3.5. Let $\Gamma$ be a graph not containing $S_{0}$. Suppose that we choose a maximal tree of $\Gamma$ and give an order as in Figure $9(\mathrm{~b})$. Then $\widetilde{R}(c)=\widetilde{R} V(c)$ for a redundant cell c in $U D_{n} \Gamma$. Consequently, $\widetilde{R}=\widetilde{R} \widetilde{V}$.

Proof. Suppose that $v_{s}$ is the smallest unblocked vertex in $c$ and $e_{s}$ is an edge of $\Gamma$ such that $\iota\left(e_{s}\right)=v_{s}$. Then $R(c)= \pm \partial W(c)+c=\sum_{e}\left(c_{e}^{\iota}-c_{e}^{\tau}\right)+V(c)$, where $c_{e}^{\iota}$ and $c_{e}^{\tau}$ are cells obtained from $W(c)$ by replacing an edge $e \neq e_{s}$ in $W(c)$ by $\iota(e)$ and $\tau(e)$, respectively. It suffices to show that $\widetilde{R}\left(c_{e}^{\iota}-c_{e}^{\tau}\right)=0$ for each edge $e \neq e_{s}$ in $c$. We use induction on the minimal integer $k$ such that $R^{k}(c)=R^{k+1}(c)$. Since $c$ is redundant, $k \geq 2$. Suppose that $k=2$. Then both $c_{e}^{l}$ and $c_{e}^{\tau}$ must be collapsible, and so $\widetilde{R}\left(c_{e}^{\iota}\right)=\widetilde{R}\left(c_{e}^{\tau}\right)=0$.

Suppose that $k \geq 3$. Among six possible relative positions of edges $e$ and $e_{s}$, $\tau\left(e_{s}\right)<\tau(e)<\iota\left(e_{s}\right)<\iota(e)$ and $\tau(e)<\tau\left(e_{s}\right)<\iota(e)<\iota\left(e_{s}\right)$ are impossible due to our choice of the maximal tree and the order of $\Gamma$ as in Figure 9(b). If $\tau\left(e_{s}\right)<$ $\iota\left(e_{s}\right)<\tau(e)<\iota(e), \tau(e)<\tau\left(e_{s}\right)<\iota\left(e_{s}\right)<\iota(e)$, or $\tau(e)<\iota(e)<\tau\left(e_{s}\right)<\iota\left(e_{s}\right)$, then both $c_{e}^{\iota}$ and $c_{e}^{\tau}$ have the order-respecting edge $e_{s}$ satisfying the hypothesis of Lemma 3.3 and so $\widetilde{R}\left(c_{e}^{\iota}\right)=\widetilde{R}\left(c_{e}^{\tau}\right)=0$. 
In the remaining case $\tau\left(e_{s}\right)<\tau(e)<\iota(e)<\iota\left(e_{s}\right)$, both $c_{e}^{\iota}$ and $c_{e}^{\tau}$ are redundant and stabilize faster than $c$. By induction, $\widetilde{R}\left(c_{e}^{\iota}\right)=\widetilde{R} V\left(c_{e}^{\iota}\right)$ and $\widetilde{R}\left(c_{e}^{\tau}\right)=\widetilde{R} V\left(c_{e}^{\tau}\right)$. If $V\left(c_{e}^{l}\right)$ has an unblocked vertex $v$ such that $\tau\left(e_{s}\right)<v<\iota\left(e_{s}\right)$, then $V\left(c_{e}^{l}\right)$ is redundant, and so $\widetilde{R} V\left(c_{e}^{\iota}\right)=\widetilde{R} V^{2}\left(c_{e}^{\iota}\right)$ by induction. Thus $\widetilde{R} V^{2}\left(c_{e}^{\iota}\right)=\widetilde{R}\left(c_{e}^{\iota}\right)$. Let $m$ be the smallest integer such that every vertex $v$ in $V^{m}\left(c_{e}^{l}\right)$ with $\tau\left(e_{s}\right)<v<$ $\iota\left(e_{s}\right)$ is blocked. Then $\widetilde{R}\left(c_{e}^{\iota}\right)=\cdots=\widetilde{R} V\left(V^{m-1}\left(c_{e}^{\iota}\right)\right)=\widetilde{R} V^{m}\left(c_{e}^{\iota}\right)=\widetilde{R} \widetilde{V}\left(c_{e}^{\iota}\right)$ by induction. Similarly, $\widetilde{R}\left(c_{e}^{\tau}\right)=\widetilde{R} \widetilde{V}\left(c_{e}^{\tau}\right)$. Thus $\widetilde{R}\left(c_{e}^{\iota}\right)=\widetilde{R} \widetilde{V}\left(c_{e}^{\iota}\right)=\widetilde{R} \widetilde{V}\left(c_{e}^{\tau}\right)=\widetilde{R}\left(c_{e}^{\tau}\right)$ by Lemma 3.4 .

Theorem 3.6. Let $\Gamma$ be a graph not containing $S_{0}$. Then there is a Morse complex $\left(M_{*}\left(U D_{n} \Gamma\right), \widetilde{\partial}\right)$ whose boundary maps are all zero, and so the $i$-th homology $H_{i}\left(M_{*}\left(U D_{n} \Gamma\right)\right)$ is the free abelian group over critical $i$-cells.

Proof. Suppose that we choose a maximal tree of $\Gamma$ and give an order as in Figure 9 (b). Let $c$ be a critical $i$-cell of $U D_{n} \Gamma$. The boundary homomorphism $\widetilde{\partial}$ of the Morse chain complex is given by $\widetilde{\partial}(c)=\widetilde{R}\left(\sum_{k=1}^{i}(-1)^{k}\left(\partial_{k}^{\iota}(c)-\partial_{k}^{\tau}(c)\right)\right)$. By Lemma 3.4 and Lemma 3.5, $\widetilde{R}\left(\partial_{k}^{\iota}(c)\right)=\widetilde{R} \widetilde{V}\left(\partial_{k}^{\iota}(c)\right)=\widetilde{R} \widetilde{V}\left(\partial_{k}^{\tau}(c)\right)=\widetilde{R}\left(\partial_{k}^{\tau}(c)\right)$. Thus $\widetilde{R}\left(\partial_{k}^{\iota}(c)-\partial_{k}^{\tau}(c)\right)=0$ for each $k$, and so $\widetilde{\partial}(c)=0$.

Let $\Gamma$ and $\Gamma^{\prime}$ be graphs. We are interested in an embedding $i: \Gamma^{\prime} \rightarrow \Gamma$ of graphs that induces homomorphisms $\tilde{i}_{*}: H_{*}\left(M_{*}\left(U D_{n^{\prime}} \Gamma^{\prime}\right)\right) \rightarrow H_{*}\left(M_{*}\left(U D_{n} \Gamma\right)\right)$ for $n^{\prime} \leq n$. If $\Gamma^{\prime}$ has a vertex of valency 1 , then we can choose maximal trees $T$ and $T^{\prime}$ of $\Gamma$ and $\Gamma^{\prime}$, respectively, and give an order on $\Gamma$ and $\Gamma^{\prime}$ as described in $\$ 2.2$ so that they satisfy the following conditions:

(1) The base vertex $0^{\prime}$ of $T^{\prime}$ has valency one in $\Gamma^{\prime}$.

(2) The edge path joining $i\left(0^{\prime}\right)$ and the base vertex 0 in $T$ passes through exactly $n-n^{\prime}$ edges, none of which are images of edges of $\Gamma^{\prime}$.

(3) The order is preserved under $i$, that is, $v_{1}<v_{2}$ iff $i\left(v_{1}\right)<i\left(v_{2}\right)$ for all vertices $v_{1}, v_{2}$ in $\Gamma^{\prime}$.

(4) The image of an edge $e$ on $\Gamma^{\prime}$ is a deleted edge on $\Gamma$ if and only if $e$ is a deleted edge on $\Gamma^{\prime}$.

By conditions (1) and (2), the embedding $i$ naturally induces an embedding $i$ : $U D_{n^{\prime}} \Gamma^{\prime} \rightarrow U D_{n} \Gamma$ for $n^{\prime} \leq n$ defined by

$$
i\left(\left\{\sigma_{1}, \sigma_{2}, \ldots, \sigma_{n^{\prime}}\right\}\right)=\left\{i\left(\sigma_{1}\right), i\left(\sigma_{2}\right), \ldots, i\left(\sigma_{n^{\prime}}\right)\right\} \cup 0_{n-n^{\prime}} .
$$

So $i$ preserves $i$-cells and, in fact, is a chain map of cubical chain complexes of $U D_{n^{\prime}} \Gamma^{\prime}$ and $U D_{n} \Gamma$. Moreover, by conditions (1), (2), and (3), $i$ and $W$ commute. So $i$ preserves critical $i$-cells and induces a chain map $\tilde{i}$ between Morse complexes, that is, $\widetilde{\partial} \tilde{i}=\tilde{i} \widetilde{\partial}: M_{i}\left(U D_{n^{\prime}} \Gamma^{\prime}\right) \rightarrow M_{i-1}\left(U D_{n} \Gamma\right)$. Thus the diagram

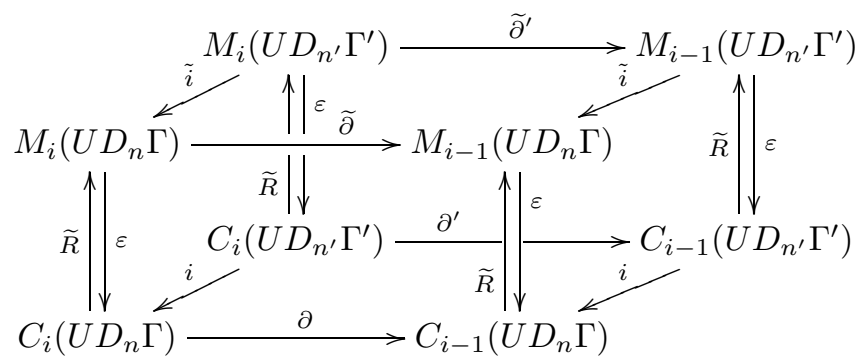


commutes. Since the chain map $\varepsilon: M_{*}\left(U D_{n} \Gamma\right) \rightarrow C_{*}\left(U D_{n} \Gamma\right)$ induces an isomorphism between homologies of two chain complexes, the diagram

$$
\begin{array}{ccc}
H_{*}\left(M_{*}\left(U D_{n^{\prime}} \Gamma^{\prime}\right)\right) \stackrel{\tilde{i}_{*}}{\longrightarrow} H_{*}\left(M_{*}\left(U D_{n} \Gamma\right)\right) \\
\cong \uparrow \widetilde{R}_{*} & \cong \downarrow \varepsilon_{*} \\
H_{*}\left(C_{*}\left(U D_{n^{\prime}} \Gamma^{\prime}\right)\right) \stackrel{i_{*}}{\longrightarrow} H_{*}\left(C_{*}\left(U D_{n} \Gamma\right)\right)
\end{array}
$$

commutes. Although the homomorphism $\tilde{i}_{*}$ may be intractable in general, it is manageable if we appropriately choose maximal trees and orders for a given embedding $i: \Gamma^{\prime} \rightarrow \Gamma$. Since $i_{*}=\varepsilon_{*} \tilde{i}_{*} \widetilde{R}_{*}$, many properties such as injectivity are shared by $i_{*}$ and $\tilde{i}_{*}$, and moreover they do not depend on the choice of maximal trees and orders.

3.2. Cohomology algebra of graphs without $S_{0}$. In [10, Farley and Sabalka employed an indirect construction to compute the cohomology algebra of the braid group of a tree with coefficients in $\mathbb{Z}_{2}$. They defined an equivalence relation on the unordered configuration space of a tree so that its quotient space is a subcomplex of a high-fold torus and the epimorphism of cohomology algebras induced from the quotient map carries the structure of the cohomology algebra of the subcomplex of the high-fold torus to that of the cohomology algebra of the braid group. In fact, they proved from this construction that the cohomology algebra of $B_{4} T_{0}$ is an exterior face algebra of a non-flag complex.

Let $\Gamma$ be a graph that contains $T_{0}$ and does not contain $S_{0}$. In this section, we take a more direct route to compute the cohomology algebra of $B_{n} \Gamma$. The two approaches start quite differently, but they end up producing essentially the same formulation. So our approach explains a motivation of the construction by Farley and Sabalka.

Let $\left(C^{i}\left(U D_{n} \Gamma ; \mathbb{Z}_{2}\right), \delta\right)$ be the cubical cellular cochain complex with coefficients in $\mathbb{Z}_{2}$ of $U D_{n} \Gamma$, and let $\left(M^{i}\left(U D_{n} \Gamma ; \mathbb{Z}_{2}\right), \tilde{\delta}\right)$ be the Morse cochain complex with coefficients in $\mathbb{Z}_{2}$ which is dual to the Morse chain complex $\left(M_{i}\left(U D_{n} \Gamma\right), \tilde{\partial}\right)$. Let $\widetilde{R}^{*}$ and $\varepsilon^{*}$ denote the dual of the chain maps $\widetilde{R}$ and $\varepsilon$ discussed in 93.1 . Consider the following commutative diagram:

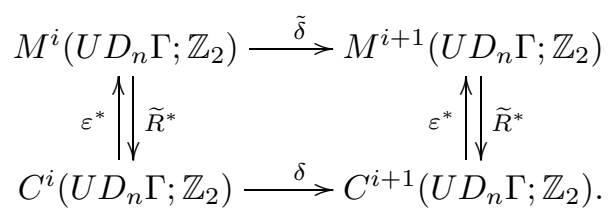

$C_{i}\left(U D_{n} \Gamma ; \mathbb{Z}_{2}\right)$ is generated by $i$-cells in $U D_{n} \Gamma$, and so $C^{i}\left(U D_{n} \Gamma ; \mathbb{Z}_{2}\right)$ is generated by their dual $c^{*}$, where $c^{*}$ is evaluated to 1 on $c$ and to 0 on other $i$-cells. Similarly, $M^{i}\left(U D_{n} \Gamma ; \mathbb{Z}_{2}\right)$ is generated by $c^{*}$ dual to critical $i$-cells $c$. Note that the domain of dual cells should be taken into account, although they are written in the same notation.

If we choose a maximal tree of $\Gamma$ and give an order as in Figure $9(\mathrm{~b})$, the boundary map $\widetilde{\partial}_{i}$ of the Morse complex $\left(M_{i}\left(U D_{n} \Gamma\right), \tilde{\partial}\right)$ is zero for all $i$ by Theorem 3.6 and so is the coboundary map $\tilde{\delta}_{i}$ of $M^{*}\left(U D_{n} \Gamma ; \mathbb{Z}_{2}\right)$. Then $c^{*}$ is a cocycle, and so $\widetilde{R}^{*}\left(c^{*}\right)$ is a cocycle of $C^{*}\left(U D_{n} \Gamma ; \mathbb{Z}_{2}\right)$. Since homology groups $H_{*}\left(M_{*}\left(U D_{n} \Gamma\right)\right)$ are free abelian groups generated by critical cells, the cohomology algebra $H^{*}\left(M_{*}\left(U D_{n} \Gamma\right) ; \mathbb{Z}_{2}\right)$ is 
generated by cohomology classes $\left[c^{*}\right]$. Since $\left(C^{*}\left(U D_{n} \Gamma\right), \delta\right)$ and $\left(M^{*}\left(U D_{n} \Gamma\right), \tilde{\delta}\right)$ are chain homotopy equivalent via $\widetilde{R}^{*}$, cohomology classes $\left[\widetilde{R}^{*}\left(c^{*}\right)\right]$ for critical cells $c$ generate the cohomology algebra $H^{*}\left(C_{*}\left(U D_{n} \Gamma\right) ; \mathbb{Z}_{2}\right)$. Therefore the algebra structure $H^{*}\left(B_{n} \Gamma ; \mathbb{Z}_{2}\right)$ is determined by the cup product $\left[\widetilde{R}^{*}\left(c^{*}\right)\right] \cup\left[\widetilde{R}^{*}\left(c^{\prime *}\right)\right]$ in $H^{*}\left(U D_{n} \Gamma ; \mathbb{Z}_{2}\right)$ for critical cells $c$ and $c^{\prime}$. One may try to compute cup products within the Morse cochain complex; then all the complications are concentrated at the coboundary map $\tilde{\delta}$. In this section, we rather want to disperse the complication so that we can quickly decide whether or not a cup product vanishes.

For an $i$-cell $a$ in $U D_{n} \Gamma, \widetilde{R}^{*}\left(c^{*}\right)(a)=c^{*}(\widetilde{R}(a))$, which equals 1 if $c$ occurs in $\widetilde{R}(a)$, or 0 otherwise. By Lemma 3.5, $\widetilde{R} \widetilde{V}(a)=\widetilde{R}(a)$. Since $\widetilde{V}(a)$ is either critical or collapsible, $\widetilde{R}(a)$ consists of one critical cell or is 0 . So $\widetilde{R}^{*}\left(c^{*}\right)=\sum_{\widetilde{R}(a)=c} a^{*}$. $\widetilde{R}(a)=\widetilde{R} \widetilde{V}(a)=c$ if and only if $\widetilde{V}(a)=c$. Therefore, $\widetilde{R}^{*}\left(c^{*}\right)=\sum_{\widetilde{V}(a)=c} a^{*}$. Note that $i$-cells $a$ satisfying $\widetilde{V}(a)=c$ can be characterized as follows: Suppose $c=$ $\left\{e_{1}, \ldots, e_{i}, v_{i+1}, \ldots, v_{n}\right\}$. Then $a$ contains the same edges as $c$ and each connected component of $\Gamma-\bar{e}_{1} \cup \cdots \cup \bar{e}_{i}$ contains the same number of vertices from $c$ as from $a$ where $\bar{e}$ denotes the closure of the open cell $e$. This precisely gives the equivalence relation considered by Farley and Sabalka in [10. Let $c=\left\{e_{1}, \ldots, e_{i}, v_{i+1}, \ldots, v_{n}\right\}$ be a critical $i$-cell and $c^{\prime}=\left\{e_{1}^{\prime}, \ldots, e_{j}^{\prime}, v_{j+1}^{\prime}, \ldots, v_{n}^{\prime}\right\}$ be a critical $j$-cell in $U D_{n} \Gamma$. Then

$$
\left[\widetilde{R}^{*}\left(c^{*}\right)\right] \cup\left[\widetilde{R}^{*}\left(c^{\prime *}\right)\right]=\left[\widetilde{R}^{*}\left(c^{*}\right) \cup \widetilde{R}^{*}\left(c^{\prime *}\right)\right]=\left[\sum_{\widetilde{V}(a)=c} a^{*} \cup \sum_{\widetilde{V}(b)=c^{\prime}} b^{*}\right] .
$$

Since $U D_{n} \Gamma$ is a cubical complex, the cup product in $H^{*}\left(U D_{n} \Gamma ; \mathbb{Z}_{2}\right)$ can be geometrically formulated as

$$
\sum_{\widetilde{V}(a)=c} a^{*} \cup \sum_{\widetilde{V}(b)=c^{\prime}} b^{*}=\sum p^{*},
$$

where the sum on the right side is taken over all $(i+j)$-cells $p$ in $U D_{n} \Gamma$ satisfying the following conditions:

(1) $e_{1}, \ldots, e_{i}, e_{1}^{\prime}, \ldots, e_{j}^{\prime}$ are edges in $p$.

(2) If an $i$-cell $a$ is an $i$-dimensional face of $p$ and if $e_{1}, \ldots, e_{i}$ are edges of $a$, then $\widetilde{V}(a)=c$.

(3) If a $j$-cell $b$ is a $j$-dimensional face of $p$ and if $e_{1}^{\prime}, \ldots, e_{j}^{\prime}$ are edges of $b$, then $\widetilde{V}(b)=c^{\prime}$.

In general, the cup product in the cohomology algebra of the Morse cochain complex can be given by

$$
\left[c^{*}\right] \cup\left[c^{\prime *}\right]=\left[\varepsilon^{*} \widetilde{R}^{*}\left(c^{*}\right)\right] \cup\left[\varepsilon^{*} \widetilde{R}^{*}\left(c^{* *}\right)\right]=\varepsilon^{*}\left(\sum p^{*}\right) .
$$

In our situation, if $\sum p^{*}$ contains a summand $q^{*}$ for a critical $(i+j)$-cell $q$, then $\sum p^{*}$ is equal to $\sum_{\widetilde{V}(x)=q} x^{*}=\left[\widetilde{R}^{*}\left(q^{*}\right)\right]$, and so $\left[c^{*}\right] \cup\left[c^{\prime *}\right]=\left[q^{*}\right]$. Otherwise, we continue to modify $\sum p^{*}$ up to coboundary until it contains such a summand or it vanishes.

In order to reduce the amount of computation, we give several sufficient conditions for a cup product to vanish, which can be direct consequences of the conditions for $\sum p^{*}$ above. If a critical $i$-cell $c=\left\{e_{1}, \ldots, e_{i}, v_{i+1}, \ldots, v_{n}\right\}$ and a critical $j$-cell $c^{\prime}=\left\{e_{1}^{\prime}, \ldots, e_{j}^{\prime}, v_{j+1}^{\prime}, \ldots, v_{n}^{\prime}\right\}$ satisfy one of the following three conditions, then the 
cup product $\left[c^{*}\right] \cup\left[c^{*}\right]=0$ since each condition violates the corresponding condition given above.

(1) There are edges $e$ in $c$ and $e^{\prime}$ in $c^{\prime}$ such that $\bar{e} \cap \bar{e}^{\prime} \neq \emptyset$.

(2) Every $i$-cell $a$ with $\widetilde{V}(a)=c$ contains neither $\iota\left(e_{\ell}^{\prime}\right)$ nor $\tau\left(e_{\ell}^{\prime}\right)$ for some $\ell=1, \ldots, j$.

(3) Every $j$-cell $b$ with $\widetilde{V}(b)=c^{\prime}$ contains neither $\iota\left(e_{\ell}\right)$ nor $\tau\left(e_{\ell}\right)$ for some $\ell=1, \ldots, i$.

There are critical cells $c$ and $c^{\prime}$ that satisfy none of the vanishing conditions, but the cup product $\left[c^{*}\right] \cup\left[c^{*}\right]$ is still 0 . It is not easy to compute the cohomology algebra of the unordered configuration space of an arbitrary graph that contains $T_{0}$ but not $S_{0}$. But it is possible to compute cohomology algebras of graph braid groups of low braid indices if a graph is simple enough such as $T_{1}, T_{2}, T_{3}^{\prime}, T_{3}^{\prime \prime}$ and $T_{3}^{\prime \prime \prime}$.

The following lemma shows that the cohomology algebras of $U D_{4} T_{1}, U D_{3} T_{2}$, $U D_{2} T_{3}^{\prime}, U D_{2} T_{3}^{\prime \prime}$, and $U D_{2} T_{3}^{\prime \prime \prime}$ are exterior face algebras of non-flag complexes. Consequently, none of the braid groups $B_{4} T_{1}, B_{3} T_{2}, B_{2} T_{3}^{\prime}, B_{2} T_{3}^{\prime \prime}$, and $B_{2} T_{3}^{\prime \prime \prime}$ is a right-angled Artin group.

Lemma 3.7. The cohomology algebra $H^{*}\left(B_{n} \Gamma ; \mathbb{Z}_{2}\right)$ is isomorphic to the exterior face algebra $\Lambda(K)$ where the braid index $n$, the graph $\Gamma$, and the simplicial complex $K$ are given by rows of the following table:

\begin{tabular}{|c|c|c|c|}
\hline$n$ & $\Gamma$ & $K$ \\
\hline 4 & $T_{1}$ & the graph in Figure 10(a) with 9 extra vertices \\
3 & $T_{2}$ & the graph in Figure 10(b) with 5 extra vertices \\
2 & $T_{3}^{\prime}$ & the graph in Figure 10(c) with 5 extra vertices \\
2 & $T_{3}^{\prime \prime}$ & the graph in Figure 10 (c) with 6 extra vertices \\
2 & $T_{3}^{\prime \prime \prime}$ & the graph in Figure 10(c) with 6 extra vertices \\
\hline
\end{tabular}

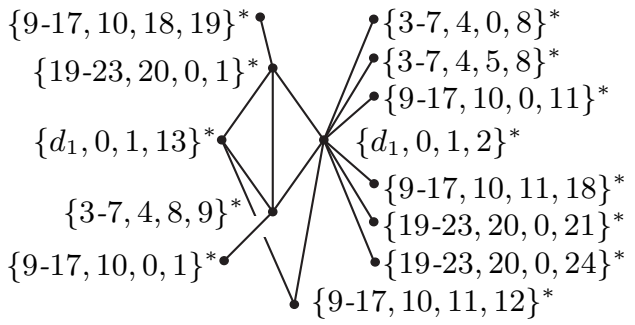

(a)

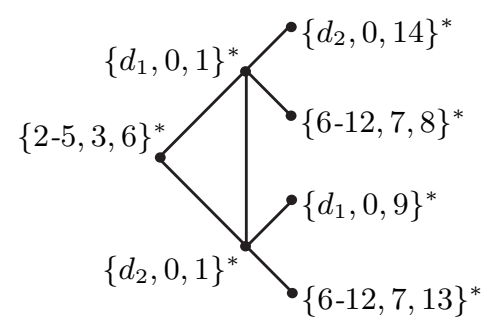

(b)

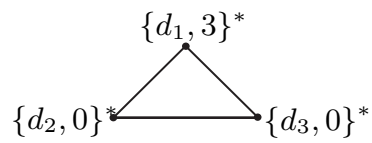

(c)

Figure 10. Complex $K$ labeled by 1-cocycles with no isolated vertices 
Proof. We only discuss $H^{*}\left(U D_{3} T_{2} ; \mathbb{Z}_{2}\right)$. The rest are similar using maximal trees and orders given in Figure 11. In the figure, vertices of valency $\geq 3$ labeled by their numbering and the base vertex 0 and deleted edges $d_{i}$ are indicated.

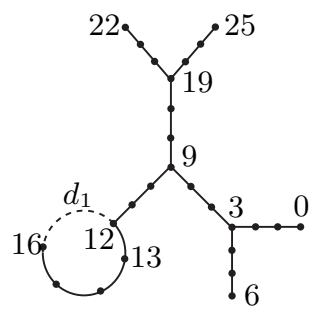

(a) $T_{1}$

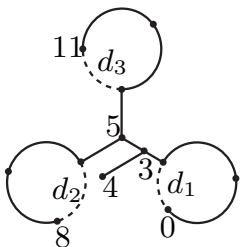

(c) $T_{3}^{\prime}$

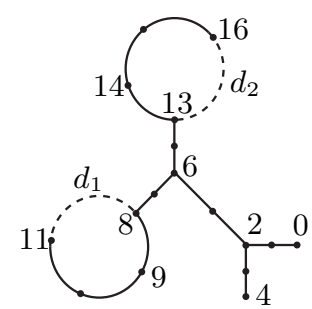

(b) $T_{2}$

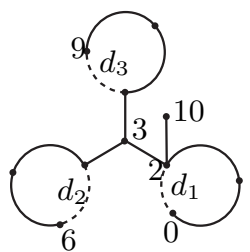

(e) $T_{3}^{\prime \prime \prime}$

FIgURE 11. Maximal trees with numbering on vertices

If we choose a maximal tree of $T_{2}$ and give an order as in Figure 11(b), then the complex $U D_{3} T_{2}$ has twelve critical 1-cells $\{2-5,3,0\},\{2-5,3,4\},\{2-5,3,6\}$, $\{6-12,7,0\},\{6-12,7,8\},\{6-12,7,13\},\left\{d_{1}, 0,1\right\},\left\{d_{1}, 0,9\right\},\left\{d_{1}, 9,10\right\},\left\{d_{2}, 0,1\right\}$, $\left\{d_{2}, 0,14\right\},\left\{d_{2}, 14,15\right\}$, and seven critical 2-cells $\left\{d_{1}, d_{2}, 0\right\},\left\{d_{1}, d_{2}, 9\right\},\left\{d_{1}, d_{2}, 14\right\}$, $\left\{2-5, d_{1}, 3\right\},\left\{2-5, d_{2}, 3\right\},\left\{6-12, d_{1}, 7\right\},\left\{6-12, d_{2}, 7\right\}$. Also, there are no critical $i$-cells for $i \geq 3$. Thus, the cohomology algebra $H^{*}\left(U D_{3} T_{2} ; \mathbb{Z}_{2}\right)$ is completely determined by the cup products of cohomology classes that are dual to critical 1-cells. Let $c=\left\{e_{1}, v_{2}, v_{3}\right\}$ and $c^{\prime}=\left\{e_{1}^{\prime}, v_{2}^{\prime}, v_{3}^{\prime}\right\}$ be critical 1-cells. Recall the formula

$$
\left[\widetilde{R}^{*}\left(c^{*}\right)\right] \cup\left[\widetilde{R}^{*}\left(c^{*}\right)\right]=\left[\sum_{\widetilde{V}(a)=c} a^{*} \cup \sum_{\widetilde{V}(b)=c^{\prime}} b^{*}\right]=\left[\sum p^{*}\right]
$$

where the sum on the right side is taken over all 2-cells $p$ in $U D_{3} T_{2}$ such that

(1) $e_{1}, e_{1}^{\prime} \in p$;

(2) $\widetilde{V}(a)=c$ for a 1-dimensional face $a$ of $p$ containing $e_{1}$;

(3) $\widetilde{V}(b)=c^{\prime}$ for a 1-dimensional face $b$ of $p$ containing $e_{1}^{\prime}$.

If $c$ and $c^{\prime}$ satisfy one of the vanishing conditions explained earlier, then the cup product vanishes. For example, consider the case $c=\{2-5,3,6\}$ and $c^{\prime}=\left\{d_{1}, 0,9\right\}$. Then $p$ must be a 2-cell that contains edges 2-5 and $d_{1}$. If $a$ is a face of $p$ containing the edge $2-5$ and $\widetilde{V}(a)=c$, then $p$ is either $\left\{2-5, d_{1}, 3\right\}$ or $\left\{2-5, d_{1}, 4\right\}$. On the 
other hand, if $b$ is a face of $p$ containing $d_{1}$ and $\widetilde{V}(b)=\left\{d_{1}, 0,9\right\}$, then $p$ is either $\left\{2-5, d_{1}, 9\right\}$ or $\left\{2-5, d_{1}, 10\right\}$. Thus no such 2-cell $p$ exists, and so $\left[\widetilde{R}^{*}\left(c^{*}\right)\right] \cup\left[\widetilde{R}^{*}\left(c^{*}\right)\right]=$ 0 and so $\left[\{2-5,3,6\}^{*}\right] \cup\left[\left\{d_{1}, 0,9\right\}^{*}\right]=0$. Similarly, the following cup products vanish:

$$
\begin{aligned}
& {\left[\{2-5,3,6\}^{*}\right] \cup\left[\{6-12,7,0\}^{*}\right], \quad\left[\{2-5,3,6\}^{*}\right] \cup\left[\left\{d_{2}, 0,14\right\}^{*}\right],} \\
& {\left[\{6-12,7,0\}^{*}\right] \cup\left[\left\{d_{1}, 0,9\right\}^{*}\right], \quad\left[\{6-12,7,13\}^{*}\right] \cup\left[\left\{d_{1}, 0,9\right\}^{*}\right] \text {, }} \\
& {\left[\{6-12,7,13\}^{*}\right] \cup\left[\left\{d_{2}, 0,14\right\}^{*}\right], \quad\left[\left\{d_{1}, 0,9\right\}^{*}\right] \cup\left[\left\{d_{2}, 0,14\right\}^{*}\right] .}
\end{aligned}
$$

If $\sum p^{*}$ contains a summand $q *$ for a critical 2-cell $q$, then $\left[c^{*}\right] \cup\left[c^{*}\right]=\left[q^{*}\right]$ as mentioned before. For example, consider the case $c=\{2-5,3,6\}$ and $c^{\prime}=\left\{d_{1}, 0,1\right\}$. Then

$$
\begin{aligned}
{\left[\widetilde{R}^{*}\left(c^{*}\right)\right] \cup\left[\widetilde{R}^{*}\left(c^{\prime *}\right)\right] } & =\left[\sum_{\substack{3 \leq i \leq 4 \\
6 \leq j \leq 16}}\{2-5, i, j\}^{*} \cup \sum_{k, \ell \in A}\left\{d_{1}, k, \ell\right\}^{*}\right] \\
& =\left[\sum_{\widetilde{V}(x)=\left\{2-5, d_{1}, 3\right\}} x^{*}\right]=\left[\widetilde{R}^{*}\left(\left\{2-5, d_{1}, 3\right\}^{*}\right)\right],
\end{aligned}
$$

where $A=\{0, \ldots, 7,12, \ldots, 16\}$. Thus $\left[\{2-5,3,6\}^{*}\right] \cup\left[\left\{d_{1}, 0,1\right\}^{*}\right]=\left[\left\{2-5, d_{1}, 3\right\}^{*}\right]$.

The following cup products are obtained similarly:

$$
\begin{aligned}
{\left[\{2-5,3,6\}^{*}\right] \cup\left[\left\{d_{2}, 0,1\right\}^{*}\right] } & =\left[\left\{2-5, d_{2}, 3\right\}^{*}\right], \\
{\left[\{6-12,7,8\}^{*}\right] \cup\left[\left\{d_{1}, 0,1\right\}^{*}\right] } & =\left[\left\{6-12, d_{1}, 7\right\}^{*}\right], \\
{\left[\{6-12,7,13\}^{*}\right] \cup\left[\left\{d_{2}, 0,1\right\}^{*}\right] } & =\left[\left\{6-12, d_{2}, 7\right\}^{*}\right], \\
{\left[\left\{d_{1}, 0,1\right\}^{*}\right] \cup\left[\left\{d_{2}, 0,1\right\}^{*}\right] } & =\left[\left\{d_{1}, d_{2}, 0\right\}^{*}\right], \\
{\left[\left\{d_{1}, 0,1\right\}^{*}\right] \cup\left[\left\{d_{2}, 0,14\right\}^{*}\right] } & =\left[\left\{d_{1}, d_{2}, 14\right\}^{*}\right], \\
{\left[\left\{d_{1}, 0,9\right\}^{*}\right] \cup\left[\left\{d_{2}, 0,1\right\}^{*}\right] } & =\left[\left\{d_{1}, d_{2}, 9\right\}^{*}\right] .
\end{aligned}
$$

If $\sum p^{*}$ contains no dual to any critical 2 -cell, then we continue to modify $\sum p^{*}$ up to coboundary until it contains such a summand or it vanishes. For the case $c=\{6-12,7,0\}$ and $c^{\prime}=\left\{d_{1}, 0,1\right\}$,

$$
\begin{aligned}
{\left[\widetilde{R}^{*}\left(c^{*}\right)\right] \cup\left[\widetilde{R}^{*}\left(c^{\prime *}\right)\right]=} & {\left[\left\{6-12, d_{1}, 0\right\}^{*}+\left\{6-12, d_{1}, 1\right\}^{*}+\left\{6-12, d_{1}, 2\right\}^{*}\right.} \\
& \left.+\left\{6-12, d_{1}, 3\right\}^{*}+\left\{6-12, d_{1}, 4\right\}^{*}+\left\{6-12, d_{1}, 5\right\}^{*}\right] .
\end{aligned}
$$

Since $\left\{6-12, d_{1}, 0\right\},\left\{6-12, d_{1}, 1\right\},\left\{6-12, d_{1}, 2\right\},\left\{6-12, d_{1}, 3\right\},\left\{6-12, d_{1}, 4\right\}$ and $\left\{6-12, d_{1}, 5\right\}$ are not critical, we consider the cochain $\alpha^{*}=\sum_{0 \leq i, j \leq 7}\left\{d_{1}, i, j\right\}^{*}$ in $C^{1}\left(U D_{3} T_{2} ; \mathbb{Z}_{2}\right)$. Then

$$
\delta\left(\alpha^{*}\right)=\widetilde{R}^{*}\left(\{6-12,7,0\}^{*}\right) \cup \widetilde{R}^{*}\left(\left\{d_{1}, 0,1\right\}^{*}\right)+\widetilde{R}^{*}\left(\{6-12,7,8\}^{*}\right) \cup \widetilde{R}^{*}\left(\left\{d_{1}, 0,1\right\}^{*}\right) .
$$

So,

$$
\left[\{6-12,7,0\}^{*}\right] \cup\left[\left\{d_{1}, 0,1\right\}^{*}\right]=\left[\{6-12,7,8\}^{*}\right] \cup\left[\left\{d_{1}, 0,1\right\}^{*}\right]=\left[\left\{6-12, d_{1}, 7\right\}^{*}\right] .
$$

For the case $c=\{6-12,7,8\}$ and $c^{\prime}=\left\{d_{1}, 0,9\right\}$,

$$
\left[\widetilde{R}^{*}\left(c^{*}\right)\right] \cup\left[\widetilde{R}^{*}\left(c^{*}\right)\right]=\left[\left\{6-12, d_{1}, 9\right\}^{*}+\left\{6-12, d_{1}, 10\right\}^{*}\right] .
$$

Since $\left\{6-12, d_{1}, 9\right\}$ and $\left\{6-12, d_{1}, 10\right\}$ are not critical, we consider the cochain $\beta^{*}=$ $\sum_{\substack{0 \leq i \leq 7 \\ 9 \leq j \leq 10}}\left\{d_{1}, i, j\right\}^{*}$ in $C^{1}\left(U D_{3} T_{2} ; \mathbb{Z}_{2}\right)$. Then $\delta\left(\beta^{*}\right)=\left\{6-12, d_{1}, 9\right\}^{*}+\left\{6-12, d_{1}, 10\right\}^{*}$. Thus $\left[\{6-12,7,8\}^{*}\right] \cup\left[\left\{d_{1}, 0,9\right\}^{*}\right]=0$. Similarly,

$$
\left[\widetilde{R}^{*}\left(\{6-12,7,13\}^{*}\right)\right] \cup\left[\widetilde{R}^{*}\left(\left\{d_{1}, 0,1\right\}^{*}\right)\right]=\left[\delta\left(\gamma^{*}\right)\right]=0
$$


for the cochain $\gamma^{*}=\sum_{12 \leq i, j \leq 16}\left\{d_{1}, i, j\right\}^{*}$ in $C^{1}\left(U D_{3} T_{2} ; \mathbb{Z}_{2}\right)$. In order to see the cohomology algebra $H^{*}\left(U D_{3} \bar{T}_{2} ; \mathbb{Z}_{2}\right)$ as an exterior face algebra of a complex, we need to change a basis by replacing $\left[\{6-12,7,0\}^{*}\right]$ by $\left[\{6-12,7,0\}^{*}\right]+\left[\{6-12,7,8\}^{*}\right]$, due to the relation that resulted from the coboundary $\delta\left(\alpha^{*}\right)$. Then

$$
\left(\left[\{6-12,7,0\}^{*}\right]+\left[\{6-12,7,8\}^{*}\right]\right) \cup\left[\left\{d_{1}, 0,1\right\}^{*}\right]=0 .
$$

Consequently, $H^{*}\left(U D_{3} T_{2} ; \mathbb{Z}_{2}\right)$ is isomorphic to an exterior face algebra of a graph in Figure 9(b) together with 5 extra vertices which is a non-flag complex. In the figure, each vertex is labeled by a 1-cocycle dual to a critical 1-cell.

It should be noted that the approach in this section cannot be used to compute the cohomology algebra of graph braid groups unless the boundary maps $\tilde{\partial}$ of the Morse chain complex are all zero and $\widetilde{R} \widetilde{V}(c)=\widetilde{R}(c)$.

3.3. Graphs that are not $T_{3}$ and contain $T_{0}$ but not $S_{0}$. Recall that for $k=0,1,2$, the set $\mathcal{G}_{k}$ consists of graphs that contain $T_{k}$ but do not contain $T_{k+1}$ and $\mathcal{G}_{3}$ consists of graphs containing $T_{3}^{\prime}, T_{3}^{\prime \prime}$ or $T_{3}^{\prime \prime \prime}$ but not $S_{0}$. If a graph $\Gamma$ is in $\bigcup_{k=0}^{3} \mathcal{G}_{k}$, we want to show $B_{n} \Gamma$ is not a right-angled Artin group. In view of Proposition 3.1 and Lemma 3.7, the only remaining ingredient is the following lemma.

Lemma 3.8. Let $\Gamma$ be a graph in $\mathcal{G}_{k}$ for some $k=0,1,2,3$. Then there is an embedding $i: P_{k} \rightarrow \Gamma$ that induces a degree-preserving epimorphism $i^{*}: H^{*}\left(B_{n} \Gamma ; \mathbb{Z}_{2}\right) \rightarrow$ $H^{*}\left(B_{n_{k}} P_{k}, \mathbb{Z}_{2}\right)$ of cohomology algebras such that $\operatorname{ker}\left(i^{*}\right)$ is generated by homogeneous elements of degree 1 and 2 , where $P_{j}=T_{j}$ for $j=0,1,2$ and $P_{3}$ is one of $T_{3}^{\prime}, T_{3}^{\prime \prime}$ and $T_{3}^{\prime \prime \prime}$, and where $n_{0}=4, n_{1}=4, n_{2}=3, n_{3}=2$ and $n \geq n_{i}$.

Proof. It is easy to find an embedding $i: P_{k} \rightarrow \Gamma$ satisfying the conditions discussed right after Theorem 3.6. Then the induced embedding $i: U D_{n_{k}} P_{k} \rightarrow U D_{n} \Gamma$ for $n \geq n_{k}$ preserves cell types and induces a chain map $\tilde{i}: M_{*}\left(U D_{n_{k}} P_{k}\right) \rightarrow$ $M_{*}\left(U D_{n} \Gamma\right)$ between Morse chain complexes. By Theorem 3.6, the homology groups $H_{*}\left(M_{*}\left(U D_{n_{k}} P_{k}\right)\right)$ and $H_{*}\left(M_{*}\left(U D_{n} \Gamma\right)\right)$ are free abelian groups that are generated by critical cells. Then the induced map $\tilde{i}_{*}: H_{*}\left(M_{*}\left(U D_{n_{k}} P_{k}\right)\right) \rightarrow H_{*}\left(M_{*}\left(U D_{n} \Gamma\right)\right)$ is injective and sends generators to generators. By the universal coefficient theorem, the induced map $\tilde{i}^{*}: H^{*}\left(M_{*}\left(U D_{n} \Gamma\right) ; \mathbb{Z}_{2}\right) \rightarrow H^{*}\left(M_{*}\left(U D_{n_{k}} P_{k}\right) ; \mathbb{Z}_{2}\right)$ of cohomology algebras is surjective and sends the generator $\left[c^{*}\right]$ to $\left[\tilde{i}^{*}\left(c^{*}\right)\right]$ if $c \in \operatorname{im}(i)$, or to 0 otherwise. Also, $\tilde{i}^{*}$ is clearly a degree-preserving algebra homomorphism.

We now show that $\operatorname{ker}\left(\tilde{i}^{*}\right)$ is generated by homogeneous elements of degree 1 and 2. Let $c$ be a critical $j$-cell in $U D_{n} \Gamma$ for $j \geq 3$. Since there is no critical $j$-cell in $U D_{n_{k}} P_{k}$ with $j \geq 3, H^{j}\left(U D_{n_{k}} P_{k}\right)=0$, and so $\left[c^{*}\right] \in \operatorname{ker}\left(\tilde{i}^{*}\right)$ since $\tilde{i}^{*}$ is degree-preserving. By induction on the degree of $c^{*}$, it suffices to show that $\left[c^{*}\right]$ is divisible by a cohomology class $\left[a^{*}\right] \in \operatorname{ker}\left(\tilde{i}^{*}\right)$ for some critical 1-cell $a$ in $U D_{n} \Gamma$. Since the embedding $i$ preserves cell type and there is no critical $j$-cell for $j \geq 3, c \notin \operatorname{im}(i)$. Thus there is either an edge or a vertex in $c$ that is not in $\operatorname{im}(i)$. If $c$ contains an edge $e^{\prime}$ that is not in $\operatorname{im}(i)$, then a 1-cell $b$ can be obtained by taking a 1-dimensional face of $c$ containing $e^{\prime}$. Since $c$ is critical, $c$ contains no order-respecting edges, and so $b$ contains no order-respecting edge either. Thus $\widetilde{V}(b)$ is a critical 1-cell. Let $a=\widetilde{V}(b)$. Then $\left[\widetilde{R}^{*}\left(c^{*}\right)\right]$ is divisible by $\left[\widetilde{R}^{*}\left(a^{*}\right)\right]$ by the construction of $b$. Thus $\left[c^{*}\right]$ is divided by $\left[a^{*}\right]$. Since $\widetilde{V}(b)$ contains the edge $e^{\prime}$, $a \notin \operatorname{im}(i)$. Therefore $\left[a^{*}\right] \in \operatorname{ker}\left(\tilde{i}^{*}\right)$. 
If $c$ contains a vertex $v^{\prime}$ that is not in $\operatorname{im}(i)$, there is an edge $e^{\prime}$ that is not orderrespecting, and $v^{\prime}$ is blocked by $e^{\prime}$. Recall that we say that a vertex $v$ is blocked by an edge $e$ in a cell $c$ if there are no vertices in $\Gamma-c$ that are smaller than $v$ and larger than an end of $e$ that is smaller than $v$. Let $b$ be a 1-dimensional face of $c$ containing $e^{\prime}$. Then $\left[\widetilde{R}\left(c^{*}\right)\right]$ is divisible by $\left[\widetilde{R}^{*}\left(a^{*}\right)\right]$, where $a=\widetilde{V}(b)$. Since $v^{\prime}$ is blocked by $e^{\prime}, v^{\prime}$ is still in the critical 1-cell $\widetilde{V}(b)$, and so $a \notin \operatorname{im}(i)$. Thus $\left[a^{*}\right] \in \operatorname{ker}\left(\tilde{i}^{*}\right)$ and $\left[c^{*}\right]$ is divisible by $\left[a^{*}\right] \in \operatorname{ker}\left(\tilde{i}^{*}\right)$.

Corollary 3.9. Using the notation of Lemma 3.8, the braid group $B_{n} \Gamma$ is not a right-angled Artin group for $n \geq n_{k}$ if a graph $\Gamma$ is in $\mathcal{G}_{k}$ for $k=0,1,2,3$.

Proof. Immediate from Proposition 3.1 together with Lemmas 3.7 and 3.8 .

3.4. Braid group of $T_{3}$. By a computation similar to those in the previous section, it is not hard to see that the cohomology algebra $H^{*}\left(B_{2} T_{3} ; \mathbb{Z}_{2}\right)$ is the exterior face algebra of the complex consisting of a triangle together with 4 extra vertices. As explained earlier, this computation cannot be carried over to the braid group over $T_{3}$ of arbitrarily large braid indices due to the non-existence of vertices of valency 1 in $T_{3}$. We introduce another tool that, in the rest of the article, will provide several necessary conditions for a group to be a right-angled Artin group.

A group $G$ is called a commutator-related group if it has a finite presentation $\left\langle x_{1}, \ldots, x_{n} \mid r_{1}, \ldots, r_{m}\right\rangle$ such that each relator $r_{j}$ belongs to the commutator subgroup $[F, F]$ of the free group $F$ generated by $x_{1}, \ldots, x_{n}$. Let $R$ be the normal subgroup of $F$ generated by $r_{1}, \ldots, r_{m}$. Then we can define a homomorphism

$$
\Phi_{G}: R /[F, R] \rightarrow[F, F] /[F,[F, F]]
$$

induced by the inclusion $R \rightarrow[F, F]$. Then $H_{2}(G)$ is identified with $R /[F, R]$ by Hopf's isomorphism, $H_{1}(G)$ is canonically identified with $F /[F, F]$, and $H_{1}(G) \wedge$ $H_{1}(G)$ is identified with $[F, F] /[F,[F, F]]$ by sending $(a[F, F]) \wedge(b[F, F])$ onto the coset $[a, b][F,[F, F]]$ for $a, b \in F$. So $\Phi_{G}$ can be regarded as a homomorphism

$$
\Phi_{G}: H_{2}(G) \rightarrow H_{1}(G) \wedge H_{1}(G)
$$

induced by $\Phi_{G}$. The following proposition says the homomorphism $\Phi_{G}$ is related to something more familiar.

Proposition 3.10 (Matei-Suciu [13]). If $G$ is a commutator-related group, then $\Phi_{G}: H_{2}(G) \rightarrow H_{1}(G) \wedge H_{1}(G)$ is the dual of the cup product $H^{1}(G) \wedge H^{1}(G) \rightarrow$ $H^{2}(G)$.

If $G$ is a right-angled Artin group, then the cohomology ring $H^{*}(G)$ is an exterior face ring of a flag complex by Proposition 3.1(1). So $H^{*}(G)$ is completely determined by the cup product $H^{1}(G) \wedge H^{1}(G) \rightarrow H^{2}(G)$. Since a right-angled Artin group is a commutator-related group and the cup product is the dual of $\Phi_{G}$, the cohomology ring $H^{*}(G)$ is completely determined by the homomorphism $\Phi_{G}$.

We show that $B_{n} T_{3}$ is a commutator-related group for $n \geq 2$ and find a presentation such that $\Phi_{G}$ sends its relators to commutators of its generators. Then we can compute the cup product $H^{1}(G) \wedge H^{1}(G) \rightarrow H^{2}(G)$ directly from the presentation and the homomorphism $\Phi_{G}$. Suppose $B_{n} T_{3}$ is a right-angled Artin group. Then we can compute the rank of $H_{3}\left(B_{n} T_{3}\right)$ by counting certain triples of generators given by the duality of Proposition 3.10 .

On the other hand, we also compute the rank of $H_{3}\left(B_{n} T_{3}\right)$ using the Morse complex of $U D_{n} T_{3}$ since $U D_{n} T_{3}$ is a $K\left(B_{n} T_{3}, 1\right)$ space. If two ranks are different, 
then we conclude that the contradiction is caused by the assumption that $B_{n} T_{3}$ is a right-angled Artin group. This strategy proves the following:

Theorem 3.11. Let $T_{3}$ be a graph in Figure 8 and let $n \geq 2$. The group $B_{n} T_{3}$ is not a right-angled Artin group.

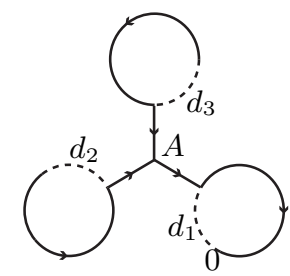

Figure 12. Maximal tree of $T_{3}$ and deleted edges

Proof. If we choose a maximal tree of $T_{3}$ and give an order as in Figure 12, the complex $U D_{n} T_{3}$ has two kinds of critical 1-cells:

$$
d_{i}(k), \quad A_{2}(\vec{a}),
$$

and two kinds of critical 2-cells: for $i \neq j$

$$
A_{2}(\vec{a}) \cup d_{i}(k), \quad d_{i}(k) \cup d_{j}(\ell),
$$

and two kinds of critical 3-cells: for $i \neq j$

$$
A_{2}(\vec{a}) \cup d_{i}(k) \cup d_{j}(\ell), \quad d_{1}(k) \cup d_{2}(\ell) \cup d_{3}(m) .
$$

Here $i, j \in\{1,2,3\}$ and $d_{i}(k)$ denotes the set consisting of $d_{i}$ together with $k$ blocked vertices from $\tau\left(d_{i}\right)$ if $i=2,3$ and $\iota\left(d_{i}\right)$ if $i=1$.

We consider the Morse complex $M_{i}\left(U D_{n} T_{3}\right)$ of $U D_{n} T_{3}$ determined by the above critical $i$-cells. By Theorem 3.6, $H_{i}\left(M_{*}\left(U D_{n} T_{3}\right)\right)$ is the free group generated by the critical $i$-cells. We first consider the number $N_{A, i, j}$ of all critical 3-cells of the form $A_{2}(\vec{a}) \cup d_{i}(k) \cup d_{j}(\ell)$ that consists of $d_{i}, d_{j}$ and $A_{2}(1,1)$ together with $n-4$ blocked vertices. Each blocked vertex is placed on $T_{3}-\left(\left\{\bar{d}_{i}, \bar{d}_{j}\right\} \cup A_{2}(1,1)\right)$ that consists of five connected components where $\bar{d}_{i}$ is the closure of $d_{i}$. Thus $N_{A, i, j}$ is the number of ways to choose $n-4$ objects from 5 objects with repetitions, and so

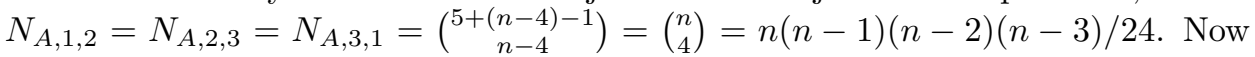
consider the number $N_{1,2,3}$ of all critical 3-cells of the form $d_{1}(k) \cup d_{2}(\ell) \cup d_{3}(m)$ that consists of $d_{1}, d_{2}$ and $d_{3}$ together with $n-3$ blocked vertices placed on $T_{3}-$ $\left\{\bar{d}_{1}, \bar{d}_{2}, \bar{d}_{3}\right\}$ consisting of four connected components. So $N_{1,2,3}=\left(\begin{array}{c}4+(n-3)-1 \\ n-3\end{array}\right)=$ $\left(\begin{array}{l}n \\ 3\end{array}\right)=n(n-1)(n-2) / 6$. Thus $\operatorname{rank}\left(H_{3}\left(B_{n} T_{3}\right)\right)=\operatorname{rank}\left(M_{3}\left(U D_{n} T_{3}\right)\right)=n(n-1)$ $(n-2)(n-3) / 8+n(n-1)(n-2) / 6$.

On the other hand, $B_{n} T_{3}$ has the presentation whose generators are the critical 1-cells and whose relators are the rewritten boundary words of critical 2-cells given 
as follows:

$$
\begin{aligned}
& \tilde{r}\left(\partial\left(A_{2}(\vec{a}) \cup d_{1}(k)\right)\right)=\left[A_{2}(\vec{a}), d_{1}(|\vec{a}|+k)\right], \\
& \tilde{r}\left(\partial\left(A_{2}(\vec{a}) \cup d_{2}(k)\right)\right) \\
& \quad=\left[\left(\Pi_{a_{2}-1}^{1}(k)\right)^{-1}\left(A_{2}\left(k+1, a_{2}\right)\right)^{-1} A_{2}\left(\vec{a}+(k+1) \vec{\delta}_{1}\right) \Pi_{a_{2}-1}^{1}(k), d_{2}(k)\right], \\
& \tilde{r}\left(\partial\left(A_{2}(\vec{a}) \cup d_{3}(k)\right)\right)=\left[A_{2}\left(\vec{a}+(k+1) \vec{\delta}_{2}\right), d_{3}(k)\right], \\
& \tilde{r}\left(\partial\left(d_{1}(k) \cup d_{2}(\ell)\right)\right)=\left[d_{1}(k+\ell+1), d_{2}(\ell)\right], \\
& \tilde{r}\left(\partial\left(d_{1}(k) \cup d_{3}(\ell)\right)\right)=\left[d_{1}(k+\ell+1), d_{3}(\ell)\right], \\
& \tilde{r}\left(\partial\left(d_{2}(k) \cup d_{3}(\ell)\right)\right)=\left[\left(\Pi_{\ell+1}^{1}(k)\right)^{-1} d_{3}(\ell) \Pi_{\ell+1}^{1}(k), d_{2}(k)\right] .
\end{aligned}
$$

Here $\vec{a}=\left(a_{1}, a_{2}\right), \Pi_{i}^{1}(k)=A_{2}(k+1, i) A_{2}(k+1, i-1) \cdots A_{2}(k+1,1)$, and $\vec{\delta}_{k}$ is the $k$-th coordinate unit vector. Thus $B_{n} T_{3}$ is a commutator-related group, and then we apply Proposition 3.10 to assert that $[a, b]$ is $\operatorname{in} \operatorname{im}\left(\Phi_{B_{n} T_{3}}\right)$ up to $[F,[F, F]]$ for generators $a, b$ of $B_{n} T_{3}$ if and only if $a^{*} \cup b^{*} \neq 0$ in $H^{2}\left(B_{n} T_{3}\right)$ for the cohomology classes $a^{*}, b^{*}$ dual to the homology classes represented by $a, b$.

Suppose that $B_{n} T_{3}$ has a presentation $\left\langle x_{1}, \ldots, x_{s} \mid r_{1}, \ldots, r_{t}\right\rangle$ such that for each $i=1, \ldots, t, \Phi_{B_{n} T_{3}}\left(r_{i}\right)$ is a commutator of generators $x_{1}, \ldots, x_{s}$ up to $[F,[F, F]]$. Then $H^{2}\left(B_{n} T_{3} ; \mathbb{Z}_{2}\right)$ is generated by $x_{i}^{*} \cup x_{j}^{*}$ for $1 \leq i, j \leq s$ and there are no relations among $x_{i}^{*} \cup x_{j}^{*}$ that are non-trivial. Let $L$ be the graph with vertices $x_{1}, \ldots, x_{s}$ such that there is an edge between $x_{i}$ and $x_{j}$ if $x_{i}^{*} \cup x_{j}^{*} \neq 0$. Then the exterior face algebra $\Lambda(L)$ is isomorphic to the cohomology algebra $H^{*}\left(B_{n} T_{3}\right)$ modulo degree 3 or higher cohomology classes.

Now suppose that $B_{n} T_{3}$ is a right-angled Artin group. Then $H^{*}\left(B_{n} T_{3}\right)$ is an exterior face algebra of a flag complex $K$. Then for the 1-skeleton $K^{1}$ of $K, \Lambda\left(K^{1}\right)$ is also isomorphic to $H^{*}\left(B_{n} T_{3}\right)$ modulo degree 3 or higher cohomology classes. As noted in the introduction of \$3, a simplicial complex is completely determined by its exterior face algebras over $\mathbb{Z}_{2}$, and so $L$ is simplicially homeomorphic to $K^{1}$. Thus the number of triangles in $L$ is equal to that in $K$. Since $\operatorname{rank}\left(H_{3}\left(B_{n} T_{3}\right)\right)$ is the number of triangles in $K^{1}, \operatorname{rank}\left(H_{3}\left(B_{n} T_{3}\right)\right)$ is equal to the number of unordered triples $\left\{x_{i}, x_{j}, x_{k}\right\}$ such that $\left[x_{i}, x_{j}\right],\left[x_{j}, x_{k}\right],\left[x_{k}, x_{i}\right] \in \operatorname{im}\left(\Phi_{B_{n} T_{3}}\right)$.

The presentation obtained from the Morse complex of $U D_{n} T_{3}$ is not yet in the form described above because

$$
\begin{aligned}
& \Phi_{B_{n} T_{3}}\left(\tilde{r}\left(\partial\left(A_{2}(\vec{a}) \cup d_{2}(k)\right)\right)\right) \\
& \quad=\left[\left(A_{2}\left(k+1, a_{2}\right)\right)^{-1} A_{2}\left(\vec{a}+(k+1) \vec{\delta}_{1}\right), d_{2}(k)\right] \quad(\bmod [F,[F, F]]),
\end{aligned}
$$

which is not a commutator of generators. So we modify the presentation by Tietze transformations.

For $a_{1}, a_{2}>0$, we introduce new generators $\bar{A}(\vec{a})$ by adding the relations $\bar{A}\left(\vec{a}+\vec{\delta}_{1}\right)=\left(A_{2}(\vec{a})\right)^{-1} A_{2}\left(\vec{a}+\vec{\delta}_{1}\right)$ and $\bar{A}\left(1, a_{2}\right)=A_{2}\left(1, a_{2}\right)$, and then eliminate the generators $A_{2}(\vec{a})$. So we obtain a presentation of $B_{n} T_{3}$ with two kinds of generators $d_{i}(k)$ and $\bar{A}(\vec{a})$, and six kinds of relators as follows:

$$
\begin{array}{ll}
{\left[\bar{A}(\vec{a}), d_{1}(|\vec{a}|+k)\right],} & {\left[\left(\bar{\Pi}_{a_{2}-1}^{1}(k)\right)^{-1} \bar{A}\left(\vec{a}+(k+1) \vec{\delta}_{1}\right) \bar{\Pi}_{a_{2}-1}^{1}(k), d_{2}(k)\right],} \\
{\left[\bar{A}\left(\vec{a}+(k+1) \vec{\delta}_{2}\right), d_{3}(k)\right],} & {\left[d_{1}(k+\ell+1), d_{2}(\ell)\right],} \\
{\left[d_{1}(k+\ell+1), d_{3}(\ell)\right],} & {\left[\left(\bar{\Pi}_{\ell+1}^{1}(k)\right)^{-1} d_{3}(\ell) \bar{\Pi}_{\ell+1}^{1}(k), d_{2}(k)\right] .}
\end{array}
$$


Here $\bar{\Pi}_{i}^{1}(k)=\bar{A}(1, i) \cdots \bar{A}(k+1, i) \bar{A}(1, i-1) \cdots \bar{A}(k+1, i-1) \cdots \bar{A}(1,1) \cdots \bar{A}(k+$ $1,1)$. Then the images of the above relators under $\Phi_{B_{n} T_{3}}$ are given as follows:

$$
\begin{aligned}
& {\left[\bar{A}(\vec{a}), d_{1}(|\vec{a}|+k)\right], \quad\left[\bar{A}\left(\vec{a}+(k+1) \vec{\delta}_{1}\right), d_{2}(k)\right],} \\
& {\left[\bar{A}\left(\vec{a}+(k+1) \vec{\delta}_{2}\right), d_{3}(k)\right], \quad\left[d_{1}(k+\ell+1), d_{2}(\ell)\right],} \\
& {\left[d_{1}(k+\ell+1), d_{3}(\ell)\right], \quad\left[d_{3}(\ell), d_{2}(k)\right] \quad(\bmod [F,[F, F]]) .}
\end{aligned}
$$

As explained earlier, if the braid group $B_{n} T_{3}$ were a right-angled Artin group, then $\operatorname{rank}\left(H_{3}\left(B_{n} T_{3}\right)\right)$ is equal to the number of unordered triples $\left\{x_{1}, x_{2}, x_{3}\right\}$ of generators such that all of the mutual commutators $\left[x_{i}, x_{j}\right]$ appear in the above images. It is sufficient to consider the following four cases:

$\left\{\bar{A}(\vec{a}), d_{1}(k), d_{2}(\ell)\right\},\left\{\bar{A}(\vec{a}), d_{2}(k), d_{3}(\ell)\right\},\left\{\bar{A}(\vec{a}), d_{1}(k), d_{3}(\ell)\right\},\left\{d_{1}(k), d_{2}(\ell), d_{3}(m)\right\}$.

Let $\bar{N}_{A, i, j}$ be the number of triples $\left\{\bar{A}(\vec{a}), d_{i}(k), d_{j}(\ell)\right\}$ in which mutual commutators are all in $\operatorname{im}\left(\Phi_{B_{n} T_{3}}\right)$. In order for the commutators $\left[\bar{A}(\vec{a}), d_{i}(k)\right],\left[d_{i}(k), d_{j}(\ell)\right]$ and $\left[\bar{A}(\vec{a}), d_{j}(\ell)\right]$ to appear in the images, the parameters must satisfy the following inequalities:

$$
\begin{aligned}
n-1 \geq k \geq|\vec{a}|, a_{1} \geq \ell+2, a_{2} \geq 1, \ell \geq 0 & \text { if }(i, j)=(1,2), \\
n-1 \geq k \geq|\vec{a}|, a_{1} \geq 1, a_{2} \geq \ell+2, \ell \geq 0 & \text { if }(i, j)=(1,3), \\
n \geq|\vec{a}|, a_{1} \geq k+2, a_{2} \geq \ell+2, k, \ell \geq 0 & \text { if }(i, j)=(2,3) .
\end{aligned}
$$

Let

$$
\begin{aligned}
n_{1}=n-k-1, n_{2}=k-|\vec{a}|, n_{3}=a_{1}-\ell-2, n_{4}=a_{2}-1 & \text { if }(i, j)=(1,2), \\
n_{1}=n-k-1, n_{2}=k-|\vec{a}|, n_{3}=a_{1}-1, n_{4}=a_{2}-\ell-2 & \text { if }(i, j)=(1,3), \\
n_{1}=n-|\vec{a}|, n_{2}=a_{1}-k-2, n_{3}=a_{1}-\ell-2, n_{4}=k & \text { if }(i, j)=(2,3) .
\end{aligned}
$$

Then we have the equation

$$
n-4=n_{1}+n_{2}+n_{3}+n_{4}+\ell, \quad n_{i}, \ell \geq 0 .
$$

Also, $\bar{N}_{A, i, j}$ is the number of non-negative integer solutions for the quintuple $\left(n_{1}, n_{2}, n_{3}, n_{4}, \ell\right)$ to the above equation. So

$$
\bar{N}_{A, i, j}=\left(\begin{array}{c}
5+(n-4)-1 \\
n-4
\end{array}\right)=n(n-1)(n-2)(n-3) / 24,
$$

and so $\bar{N}_{A, i, j}=N_{A, i, j}$.

Let $\bar{N}_{1,2,3}$ be the number of triples $\left\{d_{1}(k), d_{2}(\ell), d_{3}(m)\right\}$ such that $\left[d_{1}(k), d_{2}(\ell)\right]$, $\left[d_{2}(\ell), d_{3}(m)\right]$, and $\left[d_{1}(k), d_{3}(m)\right]$ appear in $\operatorname{im}\left(\Phi_{B_{n} T_{3}}\right)$. The following inequalities must hold:

$$
n-1 \geq k \geq \ell+1, m+1, \quad n-2 \geq \ell+m, \quad \ell, m \geq 0 .
$$

Then $\bar{N}_{1,2,3}$ is equal to the number of non-negative integer solutions for the triples $(k, \ell, m)$ to the inequalities. Recall $N_{1,2,3}=n(n-1)(n-2) / 6$. We regard $N_{1,2,3}$ as the number of subsets $\{a, b, c\} \subset\{1, \ldots, n\}$. We can assume that $a>b>c$. Define a function $f:\{\{a, b, c\}\} \rightarrow\{(k, \ell, m)\}$ as follows:

$$
f(\{a, b, c\})=\left\{\begin{array}{lr}
(a-1, b-1, c-1) & \text { if } \quad b+c \leq n, \\
(n-c, n-a, n-b) & \text { otherwise. }
\end{array}\right.
$$

Then it is easy to see that $f$ is well-defined and injective. Also, the image of $f$ does not contain $(1,0,0)$. So $\bar{N}_{1,2,3}>N_{1,2,3}$. This is a contradiction because 
$\operatorname{rank}\left(H_{3}\left(B_{n} T_{3}\right)\right)$ computed from the presentation under the assumption that $B_{n} T_{3}$ is a right-angled Artin group is larger than that obtained from the Morse complex of the configuration space.

\section{Graphs Containing $S_{0}$}

Recall the homomorphism $\Phi_{G}: R /[F, R] \rightarrow[F, F] /[F,[F, F]]$ induced by the inclusion $R \hookrightarrow[F, F]$ for a commutator-related group $G=F / R$. If $G$ is a rightangled Artin group, then $\Phi_{G}$ is injective since $\Phi_{G}$ is regarded as the dual of the cup product $H^{1}(G) \wedge H^{1}(G) \rightarrow H^{2}(G)$ which is surjective for a right-angled Artin group $G$. We state this as a proposition, together with a simple proof.

Proposition 4.1. If $G$ is a right-angled Artin group, then $\Phi_{G}$ is injective.

Proof. Suppose that $G=F / R$. Since $G$ is a right-angled Artin group, $R$ is normally generated by a collection $C$ of commutators of generators. Modulo $[F, R]$, every conjugation acts trivially on $C$ and $R /[F, R]$ is identified to the free abelian group generated by $C$. Similarly, $[F, F] /[F,[F, F]]$ is identified to the free abelian group generated by basic commutators by the Hall basis theorem. Since elements of $C$ are basic commutators, $\Phi_{G}$ is merely an inclusion under these identifications.

The converse of the proposition is not true. The braid groups of the tree $T_{0}$ and all the graphs in Figure 8 are not a right-angled Artin group, but the homomorphism $\Phi_{G}$ is injective for these groups. For example, it is not hard to see that $\Phi_{B_{n} T_{3}}$ is injective from the presentation of $B_{n} T_{3}$ given in the proof of Theorem 3.11. In fact, it is sufficient to check that $\operatorname{rank}\left(H_{2}\left(B_{n} T_{3}\right)\right)=\operatorname{rank}\left(\operatorname{im} \Phi_{B_{n} T_{3}}\right)$, since they are a free abelian group. The former rank can be obtained from the Morse complex, and the latter rank can be seen from the presentation.

In contrast, we will prove in 4.1 that the braid groups of $S_{0}$ and the $\theta$-shaped graph $\Theta$ are not a right-angled Artin group by showing that $\Phi_{G}$ is not injective.

We now consider a homomorphism between two commutator-related groups. Let $G=F / R$ and $G^{\prime}=F^{\prime} / R^{\prime}$ be commutator-related groups so that $R \subset[F, F]$ and $R^{\prime} \subset\left[F^{\prime}, F^{\prime}\right]$, and let $h: G^{\prime} \rightarrow G$ be a homomorphism. Then it is clear that the diagram

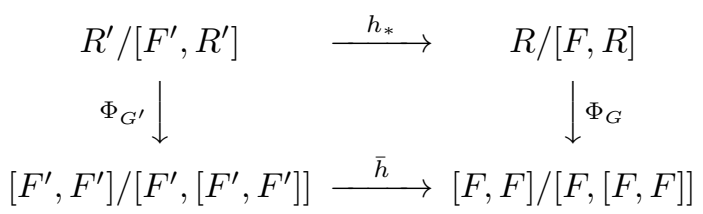

commutes, where $h_{*}$ and $\bar{h}$ are homomorphisms derived from $h$.

The assumption that $\Phi_{G^{\prime}}$ is not injective and $h_{*}$ is injective implies that $\Phi_{G}$ is not injective, and so $G$ is not a right-angled Artin group by Proposition [4.1 In order to show that $B_{n} \Gamma$ is not a right-angled Artin group for a given graph $\Gamma$, we introduce a graph $\Gamma^{\prime}$ contained in $\Gamma$ such that $\Phi_{B_{n} \Gamma^{\prime}}$ is defined and is not injective. Then we choose an embedding $i: \Gamma^{\prime} \rightarrow \Gamma$. We may assume that $B_{n} \Gamma$ is commutatorrelated; otherwise, we are done. If we show that $i$ induces an injection on the second homologies of braid groups, then $\Phi_{B_{n} \Gamma}$ is not injective, and we conclude that $B_{n} \Gamma$ is not a right-angled Artin group.

We try to apply this strategy to all graphs containing $S_{0}$. Unfortunately, there are graphs $\Gamma$ such that no embedding $i: S_{0} \rightarrow \Gamma$ induces an injection $i_{*}: H_{2}\left(B_{n} S_{0}\right)$ $\rightarrow H_{2}\left(B_{n} \Gamma\right)$. In fact, $\Theta$ is one of such examples as shown in the following lemma. 
Lemma 4.2. For $n \geq 5$, there is no embedding $i: S_{0} \rightarrow \Theta$ that induces an injection $i_{*}: H_{2}\left(B_{n} S_{0}\right) \rightarrow H_{2}\left(B_{n} \Theta\right)$.

Proof. It is sufficient to show that $\operatorname{rank}\left(H_{2}\left(B_{n} S_{0}\right)\right)>\operatorname{rank}\left(H_{2}\left(B_{n} \Theta\right)\right)$. Indeed, $\operatorname{rank}\left(H_{2}\left(B_{n} S_{0}\right)\right)=(n-3)(n-2)(n-1) / 6$, as we will see in the proof of Lemma4.4. and $\operatorname{rank}\left(H_{2}\left(B_{n} \Theta\right)\right)=(n-3)(n-2) / 2$, as we will see in the remark following Lemma 4.5 .

If $\Gamma$ contains $S_{0}$ but does not contain $\Theta$, then there is an embedding $i: S_{0} \rightarrow \Gamma$ that induces an injection $i_{*}: H_{2}\left(B_{n} S_{0}\right) \rightarrow H_{2}\left(B_{n} \Gamma\right)$, as shown in Lemma 4.22, On the other hand, if $\Gamma$ contains $\Theta$, then it seems difficult to directly show that there is an embedding $i: \Theta \rightarrow \Gamma$ that induces an injection $i_{*}: H_{2}\left(B_{n} \Theta\right) \rightarrow H_{2}\left(B_{n} \Gamma\right)$. Fortunately, $\Phi_{B_{n} \Theta}$ is the trivial map, as shown in Lemma 4.5 and therefore in order to show that $\Phi_{B_{n} \Gamma}$ is not injective, it suffices to construct an embedding $i: \Theta \rightarrow \Gamma$ that induces a non-trivial homomorphism $i_{*}: H_{2}\left(B_{n} \Theta\right) \rightarrow H_{2}\left(B_{n} \Gamma\right)$, which is shown in Lemma 4.23 under the extra assumption that $\Gamma$ is planar.

For non-planar graphs, we use yet another method to prove that non-planar graph braid groups are not a right-angled Artin group. In 4.4 it will be shown that for a non-planar graph $\Gamma$ and $n \geq 2, H_{1}\left(B_{n} \Gamma\right)$ always has a torsion, and so $B_{n} \Gamma$ cannot be a right-angled Artin group.

Consequently, the following theorem is proved by combining results in this section.

Theorem 4.3. If a graph $\Gamma$ contains $S_{0}$, then $B_{n} \Gamma$ is not a right-angled Artin group for $n \geq 5$.

To obtain the lemmas in 4.3 and $\$ 4.4$, it is necessary to extract certain information on boundary homomorphisms in Morse complexes. Since these computations are lengthy and rather technical, they are separately presented in 4.2 .

4.1. Braid groups of $S_{0}$ and $\Theta$. In Example 2.3. $B_{4} S_{0}$ was shown to be a rightangled Artin group. One can show that $B_{3} \Theta$ is also a right-angled Artin group. In this section, we show that $\Phi_{B_{n} S_{0}}\left(\Phi_{B_{n} \Theta}\right)$ is not injective for $n \geq 5(n \geq 4$, respectively). By Proposition 4.1] this implies the braid groups of $S_{0}$ and $\Theta$ are not a right-angled Artin group for the higher braid indices.

Lemma 4.4. For $n \geq 5, \Phi_{B_{n} S_{0}}$ is not injective, and so $B_{n} S_{0}$ is not a right-angled Artin group.

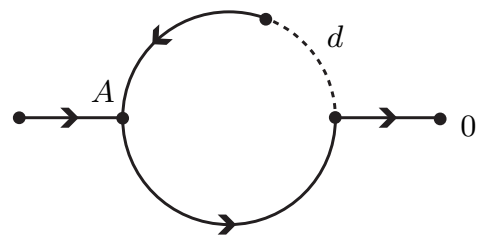

FiguRE 13. Maximal tree and order of $S_{0}$

Proof. Choose a maximal tree of $S_{0}$ and give an order as in Figure 13, Let $d(k)$ denote the set consisting of the deleted edge $d$ together with $k$ vertices blocked by $\tau(d)$. The configuration space $U D_{n} S_{0}$ has two kinds of critical 1-cells, $d(k), A_{2}(a, b)$ for $0 \leq k \leq n-1,1 \leq a, b$, and $a+b \leq n$, critical 2-cells $A_{2}(a, b) \cup d(\ell)$ for $1 \leq a, b$, 
$0 \leq \ell$, and $\ell+a+b \leq n-1$, and no critical $i$-cells for $i \geq 3$. We obtain relations by rewriting the boundaries of critical 2-cells. That is,

$$
\tilde{r}\left(\partial\left(A_{2}(a, b) \cup d(\ell)\right)\right)=(d(\ell+a+b))\left(A_{2}(a, b)\right)(d(\ell+a+b))^{-1}\left(A_{2}(a, b+1)\right)^{-1} .
$$

When $\ell+a+b=n-1$, we have the relations

$$
A_{2}(a, b+1)=d(n-1) A_{2}(a, b) d(n-1)^{-1} .
$$

Applying Tietze transformations on $A_{2}(a, b+1)=d(n-1)^{b} A_{2}(a, 1) d(n-1)^{-b}$, we eliminate the generators $A_{2}(a, b+1)$ for $b \geq 1$. For each $\ell+a+b \leq n-2$, the boundary word is rewritten as $\left[d(n-1)^{-b} d(\ell+a+b) d(n-1)^{b-1}, A_{2}(a, 1)\right]$. Let $m=$ $\ell+a+b$. We introduce new generators $\bar{d}(m)$ by adding the relations $\bar{d}(m)=(d(n-$ $1))^{-1} d(m)$ and then eliminating the generators $d(m)$. We obtain a presentation of $B_{n} S_{0}$ whose generators are $\bar{d}(0), \ldots, \bar{d}(n-2), d(n-1), A_{2}(1,1), \ldots, A_{2}(n-1,1)$ and whose relators are $\left[d(n-1)^{1-b} \bar{d}(m) d(n-1)^{b-1}, A_{2}(a, 1)\right]$ for $1 \leq a, b$ and $2 \leq a+b \leq m \leq n-2$. Thus $B_{n} S_{0}$ is a commutator-related group, and so the homomorphism $\Phi_{B_{n} S_{0}}$ is defined. Also,

$\Phi_{B_{n} S_{0}}\left(\left[d(n-1)^{1-b} \bar{d}(m) d(n-1)^{b-1}, A_{2}(a, 1)\right]\right)=\left[\bar{d}(m), A_{2}(a, 1)\right] \quad(\bmod [F,[F, F]])$, where $F$ is the free group on the set of generators. Since $b$ becomes irrelevant, we set $b=1$ to enumerate the number of commutators $\left[\bar{d}(m), A_{2}(a, 1)\right]$. Then we have $2 \leq a+1 \leq m \leq n-2$. The number of pairs $(a, m)$ satisfying the inequalities is $(n-2)(n-3) / 2$, which is equal to $\operatorname{rank}\left(\operatorname{im} \Phi_{B_{n} S_{0}}\right)$.

On the other hand, the Morse chain complex of $U D_{n} S_{0}$ looks like

$$
0 \longrightarrow M_{2}\left(U D_{n} S_{0}\right) \stackrel{\widetilde{\partial}}{\longrightarrow} M_{1}\left(U D_{n} S_{0}\right) \stackrel{0}{\longrightarrow} \mathbb{Z} .
$$

$\operatorname{Then} \operatorname{rank}\left(H_{2}\left(B_{n} S_{0}\right)\right)=\operatorname{rank}\left(M_{2}\left(U D_{n} S_{0}\right)\right)-\operatorname{rank}(\operatorname{im} \widetilde{\partial})$. Since $\widetilde{\partial}\left(A_{2}(a, b) \cup d(\ell)\right)=$ $A_{2}(a, b+1)-A_{2}(a, b), \ell$ is irrelevant. We set $\ell=0$. Then $\operatorname{im} \widetilde{\partial}$ is generated by $A_{2}(a, b+1)-A_{2}(a, b)$ for $1 \leq a, b$ and $a+b \leq n-1$. The number of pairs $(a, b)$ satisfying the inequalities is $(n-1)(n-2) / 2$, which is $\operatorname{rank}(\operatorname{im} \widetilde{\partial})$. Also, $\operatorname{rank}\left(M_{2}\left(U D_{n} S_{0}\right)\right)$ is equal to the number of triples $(a, b, \ell)$ satisfying $a, b \geq 1$, $\ell \geq 0$, and $a+b+\ell \leq n-1$. So $\operatorname{rank}\left(M_{2}\left(U D_{n} S_{0}\right)\right)=n(n-1)(n-2) / 6$. Thus $\operatorname{rank}\left(H_{2}\left(B_{n} S_{0}\right)\right)=(n-1)(n-2)(n-3) / 6$.

Consequently, since $(n-2)(n-3) / 2<(n-1)(n-2)(n-3) / 6$ for $n \geq 5, \Phi_{B_{n} S_{0}}$ cannot be injective.

Lemma 4.5. For $n \geq 4, \Phi_{B_{n} \Theta}$ is the trivial map. However, $H_{2}\left(B_{n} \Theta\right)$ is not trivial, and so $B_{n} \Theta$ is not a right-angled Artin group.

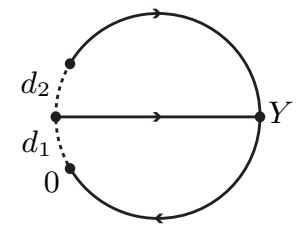

Figure 14. Maximal tree and order of $\Theta$

Proof. Choose a maximal tree $\Theta$ and give an order as in Figure 14. Then $U D_{n} \Theta$ has two kinds of critical 1-cells, $d_{\ell}, Y_{2}(a, b)$ for $1 \leq a, b, a+b \leq n, \ell=1,2$, critical 
2-cells $Y_{2}(a, b) \cup d_{\ell}$ for $1 \leq a, b, a+b \leq n-1, \ell=1,2$, and no critical $i$-cells for $i \geq 3$.

There are two types of relations obtained by rewriting boundary words of critical 2-cells as follows:

$$
\begin{aligned}
& Y_{2}(a+1, b)=\Pi_{b}^{1} d_{1} Y_{2}(a, b) d_{1}^{-1}\left(\Pi_{b-1}^{1}\right)^{-1}, \\
& Y_{2}(a, b+1)=d_{2}\left(\Pi_{b}^{1}\right)^{-1} Y_{2}(a+1, b) \Pi_{b-1}^{1} d_{2}^{-1}=d_{2} d_{1} Y_{2}(a, b)\left(d_{2} d_{1}\right)^{-1},
\end{aligned}
$$

where $\Pi_{k}^{1}=Y_{2}(1, k) Y_{2}(1, k-1) \cdots Y_{2}(1,1)$. Using the relations

$$
\begin{aligned}
Y_{2}(a+1,1) & =Y_{2}(1,1) d_{1} Y_{2}(a, 1) d_{1}^{-1}=\left(Y_{2}(1,1) d_{1}\right)^{a} Y_{2}(1,1) d_{1}^{-a}, \\
Y_{2}(a, b+1) & =\left(d_{2} d_{1}\right)^{b} Y_{2}(a, 1)\left(d_{2} d_{1}\right)^{-b} \\
& =\left(d_{2} d_{1}\right)^{b}\left(Y_{2}(1,1) d_{1}\right)^{a-1} Y_{2}(1,1) d_{1}^{1-a}\left(d_{2} d_{1}\right)^{-b},
\end{aligned}
$$

we eliminate the generators $Y_{2}(a, b)$ except $Y_{2}(1,1)$. Then there are three generators $d_{1}, d_{2}$, and $Y_{2}(1,1)$, and one type of relator as follows: For $1 \leq a, 2 \leq b$, and $a+b \leq n-1$,

$$
\left[d_{1}^{-1}\left\{\left(d_{2} d_{1}\right)^{-1} Y_{2}(1,1)\right\}^{b-1} d_{2}^{-1}\left(d_{2} d_{1}\right)^{b},\left(Y_{2}(1,1) d_{1}\right)^{a} d_{1}^{-a}\right] .
$$

Thus $B_{n} \Theta$ is a commutator-related group, and we can consider $\Phi_{B_{n} \Theta}$. It is easy to see that $\Phi_{B_{n} \Theta}$ annihilates the relations above. So $\operatorname{rank}\left(\operatorname{im} \Phi_{B_{n} \Theta}\right)=0$. But $H_{2}\left(B_{n} \Theta\right)$ is not trivial because there are commutator relators for $n \geq 4$. Thus $\operatorname{rank}\left(H_{2}\left(B_{n} \Theta\right)\right) \neq 0$ and $\Phi_{B_{n} \Theta}$ cannot be injective.

Remark. With not much extra work, we can now compute $\operatorname{rank}\left(H_{2}\left(B_{n} \Theta\right)\right)$. Since $B_{n} \Theta$ is a commutator-related group over three generators as in the proof of Lemma 4.5 $\operatorname{rank}\left(H_{1}\left(B_{n} \Theta\right)\right)=3$. Since $\operatorname{rank}\left(M_{1}\left(U D_{n} \Theta\right)\right)=n(n-1) / 2+2$,

$$
\operatorname{rank}\left(\operatorname{im}\left(\widetilde{\partial}_{2}\right)\right)=\operatorname{rank}\left(M_{1}\left(U D_{n} \Theta\right)\right)-\operatorname{rank}\left(H_{1}\left(B_{n} \Theta\right)\right)=(n-2)(n+1) / 2 .
$$

Since $\operatorname{rank}\left(M_{2}\left(U D_{n} \Theta\right)\right)=(n-1)(n-2)$,

$$
\operatorname{rank}\left(\operatorname{ker}\left(\widetilde{\partial}_{2}\right)\right)=\operatorname{rank}\left(M_{2}\left(U D_{n} \Theta\right)\right)-\operatorname{rank}\left(\operatorname{im}\left(\widetilde{\partial}_{2}\right)\right)=(n-2)(n-3) / 2 .
$$

Since $M_{3}\left(U D_{n} \Theta\right)=0$,

$$
\operatorname{rank}\left(H_{2}\left(B_{n} \Theta\right)\right)=\operatorname{rank}\left(\operatorname{ker}\left(\widetilde{\partial}_{2}\right)\right)=(n-2)(n-3) / 2 .
$$

4.2. Boundary homomorphisms in Morse complexes. In this section we will discuss the behavior of boundary homomorphisms in Morse chain complexes. In general, it is not easy to fully compute boundary homomorphisms for Morse complexes of even small graphs such as $K_{5}$. One of the reasons is that there are too many kinds of critical cells. Another reason is that Morse complexes heavily depend on geometric information such as choices of maximal trees and orderings. To show that the first homology of a non-planar braid group has a torsion, we need to understand the second boundary homomorphism of its Morse complex under a particular choice of a maximal tree. To show that braid groups of planar graphs that contain $S_{0}$ are not right-angled Artin groups, we need to look into certain images under the third boundary homomorphism.

Recall that $\widetilde{\partial}=\widetilde{R} \partial$ and that the image of an $i$-cell $c$ in $U D_{n} \Gamma$ under $\partial$ is the sum of $i$ pairs of faces of $c$ in opposite sign. So if the images of a pair of faces under $\widetilde{R}$ are identical, then this pair has no contribution in $\widetilde{\partial}(c)$. This is why $\widetilde{\partial}$ for any graph not containing $S_{0}$ is trivial, as shown in $\$ 3.1$. We introduce useful properties for $\widetilde{R}$ before discussing images under $\widetilde{\partial}$. 
Let $c$ be a redundant $i$-cell in $U D_{n} \Gamma, v$ be an unblocked vertex in $c$ and $e$ be the edge in $T$ starting from $v$. The vertex $v$ is said to be simply unblocked if there is no vertex $w$ that is either in $c$ or is an end vertex of an edge in $c$ and satisfies $\tau(e)<w<\iota(e)$. Let $W_{e}(c)\left(V_{e}(c)\right.$, respectively) denote the $(i+1)$-cell (the $i$-cell) obtained from $c$ by replacing $v$ by $e$ (by $\tau(e))$.

Lemma 4.6. Suppose a redundant cell $c$ has a simply unblocked vertex $v$ and $e$ is the edge starting from $v$. Then $\widetilde{R}(c)=\widetilde{R} V_{e}(c)$.

Proof. Every cell $c_{j}$ except $c$ and $V_{e}(c)$ that is a summand in $\partial W_{e}(c)$ has an order-respecting edge $e$ starting from $v$ that vacuously satisfies the hypothesis of Lemma 3.3. Thus $\widetilde{R}\left(c_{j}\right)=0$ as well as $\widetilde{R}\left(W_{e}(c)\right)=0$. Then

$$
0=\widetilde{\partial} \widetilde{R}\left(W_{e}(c)\right)=\widetilde{R} \partial\left(W_{e}(c)\right)=\widetilde{R}\left( \pm\left(c-V_{e}(c)\right)+\sum_{j} c_{j}\right) .
$$

Thus $\widetilde{R}(c)=\widetilde{R} V_{e}(c)$.

Since $\widetilde{\partial}=\widetilde{R} \partial$, the computation of $\widetilde{\partial}(c)$ for a cell $c$ in $U D_{n} \Gamma$ involves numerous rewriting of $\widetilde{R}$, and so it is important to have an efficient way to compute $\widetilde{R}$. Our strategy is basically to repeat the following two steps:

(I) Via repeated applications of this lemma to the smallest simply unblocked vertex, we may replace any redundant cell $c$ by another redundant cell $c^{\prime}$ such that $\widetilde{R}(c)=\widetilde{R}\left(c^{\prime}\right)$ and $c^{\prime}$ has no simply unblocked vertices.

(II) Compute $R\left(c^{\prime}\right)$, that is, replace $c^{\prime}$ by the alternating sum of faces of $W\left(c^{\prime}\right)$ other than $c^{\prime}$. Go back to step (I) for each redundant cell in the sum.

We now consider the relationship between the choice of maximal tree and the dimension of the corresponding Morse complex. An edge in a critical cell can be either a deleted edge or a non-order-respecting edge together with a blocked vertex near a vertex of valency $\geq 3$. Thus the dimension of the Morse complex over a graph $\Gamma$ under the choice of maximal tree $T$ is bounded above by

$$
\min \left\{n, N_{d}+\left\lfloor\left(n-N_{d}\right) / 2\right\rfloor, N_{d}+N_{v}\right\}
$$

where $n$ is the braid index, $N_{d}$ is the number of deleted edges in $\Gamma$, and $N_{v}$ is the number of vertices of valency $\geq 3$ in $T$.

When the braid index is relatively large, we had better obtain a maximal tree by deleting edges incident to a vertex of valency $\geq 3$ in order to achieve the minimal dimension of a Morse complex. This is how we chose the maximal trees in Lemma 4.4 and Lemma 4.5 .

However, this choice is not so wise when we consider the second boundary homomorphism in Morse complexes of non-planar graphs because deleted edges may block other vertices, and this makes the rewriting process extremely complicated. Thus for a non-planar graph $\Gamma$, we will choose a maximal tree $T$ by deleting edges whose ends are of valency two in $\Gamma$. By choosing a planar embedding of $T$, vertices of $\Gamma$ are ordered and $U D_{n} \Gamma$ collapses to a Morse complex as explained in 2.2 . Then there are no vertices blocked by deleted edges due to the construction of $T$ and there are three kinds of critical 2-cells $A_{k}(\vec{a}) \cup B_{\ell}(\vec{b}), A_{k}(\vec{a}) \cup d$, and $d \cup d^{\prime}$, where $A$ and $B$ are vertices with valency $\geq 3$ and where $d$ and $d^{\prime}$ are deleted edges. Remember that we are omitting the $0_{s}$ part, that is, a sequence of $s$ vertices blocked by the base vertex. We note that a critical 2-cell $A_{k}(\vec{a}) \cup B_{\ell}(\vec{b})$ is contained in the 
tree $T$ and vanishes under $\widetilde{\partial}$ as shown in [8] or in Theorem 3.6. In 4 4.4, we need to know the image of critical 2-cells $d \cup d^{\prime}$ under $\widetilde{\partial}: M_{2}\left(U D_{n} \Gamma\right) \rightarrow M_{1}\left(U D_{n} \Gamma\right)$. To help understand this, we first look at an example.

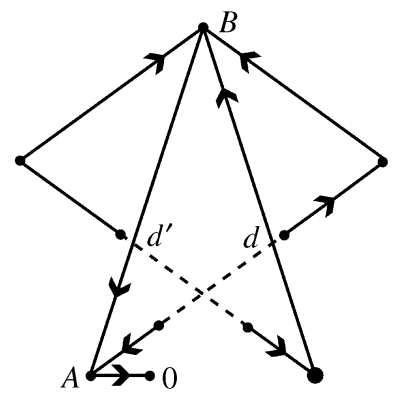

FiguRE 15. Graph containing $c=d \cup d^{\prime}$

Example 4.7. Let $\Gamma$ be a graph in Figure 15 and let a maximal tree and an order be given as the figure. As always, we assume $\Gamma$ is subdivided sufficiently according to the braid index. We want to compute $\widetilde{\partial}(c)$ for the 2-cell $c=d \cup d^{\prime}$ in $M_{2}\left(U D_{n} \Gamma\right)$.

Since $\iota\left(d^{\prime}\right)<\iota(d)$, using Lemma 4.6 we have

$$
\begin{aligned}
\widetilde{\partial}(c) & =\widetilde{R}\left(d^{\prime} \cup \iota(d)\right)-\widetilde{R}\left(d^{\prime} \cup \tau(d)\right)-\widetilde{R}\left(d \cup \iota\left(d^{\prime}\right)\right)+\widetilde{R}\left(d \cup \tau\left(d^{\prime}\right)\right) \\
& =\widetilde{R}\left(d^{\prime} \cup A(0,1)\right)-\widetilde{R}\left(d^{\prime} \cup B(0,1,0)\right)-\widetilde{R}(d \cup B(0,0,1))+d .
\end{aligned}
$$

Since $d^{\prime} \cup A_{2}(0,1)$ is collapsible, using Lemma 4.6, we have

$$
\begin{aligned}
0 & =\widetilde{\partial} \widetilde{R}\left(d^{\prime} \cup A_{2}(0,1)\right)=\widetilde{R} \partial\left(d^{\prime} \cup A_{2}(0,1)\right) \\
& =\widetilde{R}\left(d^{\prime} \cup A(0,1)-d^{\prime} \cup\{A\}-A_{2}(0,1) \cup \iota\left(d^{\prime}\right)+A_{2}(0,1) \cup \tau\left(d^{\prime}\right)\right) \\
& =\widetilde{R}\left(d^{\prime} \cup A(0,1)\right)-d^{\prime}-A_{2}(1,1)+A_{2}(1,1),
\end{aligned}
$$

and so $\widetilde{R}\left(d^{\prime} \cup A(0,1)\right)=d^{\prime}$. Similarly,

$$
\begin{aligned}
0 & =\widetilde{\partial} \widetilde{R}\left(d^{\prime} \cup B_{2}(0,1,0)\right)=\widetilde{R}\left(\partial\left(d^{\prime} \cup B_{2}(0,1,0)\right)\right) \\
& =\widetilde{R}\left(B_{2}(0,1,0) \cup \iota\left(d^{\prime}\right)-B_{2}(0,1,0) \cup \tau\left(d^{\prime}\right)-d^{\prime} \cup B(0,1,0)+d^{\prime} \cup\{B\}\right) \\
& =\widetilde{R}\left(B_{2}(0,1,0) \cup \iota\left(d^{\prime}\right)\right)-B_{2}(1,1,0)-\widetilde{R}\left(d^{\prime} \cup B(0,1,0)\right)+d^{\prime} .
\end{aligned}
$$

So $\widetilde{R}\left(d^{\prime} \cup B(0,1,0)\right)=d^{\prime}-B_{2}(1,1,0)$ since $B_{2}(0,1,0) \cup \iota\left(d^{\prime}\right)$ is collapsible. Then

$$
\begin{aligned}
0 & =\widetilde{\partial} \widetilde{R}\left(d \cup B_{3}(0,0,1)\right)=\widetilde{R}\left(\partial\left(d \cup B_{3}(0,0,1)\right)\right) \\
& =\widetilde{R}\left(B_{3}(0,0,1) \cup \iota(d)-B_{3}(0,0,1) \cup \tau(d)-d \cup B(0,0,1)+d \cup\{B\}\right) \\
& =\widetilde{R}\left(B_{3}(0,0,1) \cup \iota(d)\right)-B_{3}(0,1,1)-\widetilde{R}(d \cup B(0,0,1))+d,
\end{aligned}
$$

so $\widetilde{R}(d \cup B(0,0,1))=d-B_{3}(0,1,1)$ since $B_{3}(0,0,1) \cup \iota(d)$ is collapsible. Consequently, we have

$$
\widetilde{\partial}(c)=B_{2}(1,1,0)+B_{3}(0,1,1) .
$$


In order to give a general formula for $\widetilde{\partial}\left(d \cup d^{\prime}\right)$, we classify them into several cases depending on the relative locations of end vertices of deleted edges. The following notation are handy for this purpose and will be used later to compute the boundary of critical 3-cells. For each vertex $v$ in $\Gamma$, there is a unique edge path $\gamma_{v}$ from $v$ to the base vertex 0 in the tree $T$. For vertices $v, w$ in $\Gamma, v \wedge w$ denotes the vertex that is the first intersection between $\gamma_{v}$ and $\gamma_{w}$. Obviously, $v \wedge w \leq v$ and $v \wedge w \leq w$. For a vertex $v$ of valency $\mu$, we assign $0,1, \ldots, \mu-1$ to the branches incident to $v$ clockwise starting from the branch joined to the base vertex. Then $g(v, w)$ denotes the number assigned to the branch of $v$ containing $w$. In the case of $v$ with valency two, $g(v, w)=1$ if $v=w \wedge v$ and $g(v, w)=0$ if $v>w \wedge v$.

Although the following lemma will be used later in 4.4 we present it here in order to keep mechanical notation and ideas in one place. Also, its proof is somewhat helpful in understanding the rest of this technical section.

Lemma 4.8. Suppose that a Morse complex is obtained by choosing a maximal tree $T$ of a (non-planar) graph $\Gamma$ so that both ends of every deleted edge have valency two in $\Gamma$. For a critical 2-cell $d \cup d^{\prime}$ in $M_{2}\left(U D_{n} \Gamma\right)$ such that $d$ and $d^{\prime}$ are deleted edges with $\tau(d)<\tau\left(d^{\prime}\right)$, let $A=\tau\left(d^{\prime}\right) \wedge \tau(d), B=\tau\left(d^{\prime}\right) \wedge \iota(d), C=\iota\left(d^{\prime}\right) \wedge \tau(d)$ and $D=\iota\left(d^{\prime}\right) \wedge \iota(d)$. Then the images under the boundary map are as follows:

(1) If $\tau(d)<\iota(d)<\tau\left(d^{\prime}\right)<\iota\left(d^{\prime}\right)$, then

$$
\begin{aligned}
\widetilde{\partial}\left(d \cup d^{\prime}\right)= & A_{g\left(A, \tau\left(d^{\prime}\right)\right)}\left(\vec{\delta}_{g\left(A, \tau\left(d^{\prime}\right)\right)}+\vec{\delta}_{g(A, \tau(d))}\right) \\
& -B_{g\left(B, \tau\left(d^{\prime}\right)\right)}\left(\vec{\delta}_{g\left(B, \tau\left(d^{\prime}\right)\right)}+\vec{\delta}_{g(B, \iota(d))}\right) \\
& -C_{g\left(C, \iota\left(d^{\prime}\right)\right)}\left(\vec{\delta}_{g\left(C, \iota\left(d^{\prime}\right)\right)}+\vec{\delta}_{g(C, \tau(d))}\right) \\
& +D_{g\left(D, \iota\left(d^{\prime}\right)\right)}\left(\vec{\delta}_{g\left(D, \iota\left(d^{\prime}\right)\right)}+\vec{\delta}_{g(D, \iota(d))}\right) .
\end{aligned}
$$

(2) If $\tau(d)<\tau\left(d^{\prime}\right)<\iota\left(d^{\prime}\right)<\iota(d)$, then

$$
\begin{aligned}
\widetilde{\partial}\left(d \cup d^{\prime}\right)= & -A_{g\left(A, \tau\left(d^{\prime}\right)\right)}\left(\vec{\delta}_{g\left(A, \tau\left(d^{\prime}\right)\right)}+\vec{\delta}_{g(A, \tau(d))}\right) \\
& -B_{g(B, \iota(d))}\left(\vec{\delta}_{g\left(B, \tau\left(d^{\prime}\right)\right)}+\vec{\delta}_{g(B, \iota(d))}\right) \\
& +C_{g\left(C, \iota\left(d^{\prime}\right)\right)}\left(\vec{\delta}_{g\left(C, \iota\left(d^{\prime}\right)\right)}+\vec{\delta}_{g(C, \tau(d))}\right) \\
& +D_{g(D, \iota(d))}\left(\vec{\delta}_{g\left(D, \iota\left(d^{\prime}\right)\right)}+\vec{\delta}_{g(D, \iota(d))}\right) .
\end{aligned}
$$

(3) If $\tau(d)<\tau\left(d^{\prime}\right)<\iota(d)<\iota\left(d^{\prime}\right)$, then

$$
\begin{aligned}
\widetilde{\partial}\left(d \cup d^{\prime}\right)= & A_{g\left(A, \tau\left(d^{\prime}\right)\right)}\left(\vec{\delta}_{g\left(A, \tau\left(d^{\prime}\right)\right)}+\vec{\delta}_{g(A, \tau(d))}\right) \\
& +B_{g(B, \iota(d))}\left(\vec{\delta}_{g\left(B, \tau\left(d^{\prime}\right)\right)}+\vec{\delta}_{g(B, \iota(d))}\right) \\
& -C_{g\left(C, \iota\left(d^{\prime}\right)\right)}\left(\vec{\delta}_{g\left(C, \iota\left(d^{\prime}\right)\right)}+\vec{\delta}_{g(C, \tau(d))}\right) \\
& +D_{g\left(D, \iota\left(d^{\prime}\right)\right)}\left(\vec{\delta}_{g\left(D, \iota\left(d^{\prime}\right)\right)}+\vec{\delta}_{g(D, \iota(d))}\right),
\end{aligned}
$$

where there may be 1-cells that are not critical and should be ignored.

Proof. The boundary of a critical 2-cell made of only deleted edges consists of four 1-cells of the form $d \cup\{v\}$, where $d$ is a deleted edge and $v$ is a vertex. Since both ends of every deleted edge are of valency two in $\Gamma, v$ is a vertex of valency one in the tree $T$. Let $E=\iota(d) \wedge v$ and $F=\tau(d) \wedge v$. Then

$$
\widetilde{R}(d \cup\{v\})=d+E_{g(E, v)}\left(\vec{\delta}_{g(E, v)}+\vec{\delta}_{g(E, \iota(d))}\right)-F_{g(F, v)}\left(\vec{\delta}_{g(F, v)}+\vec{\delta}_{g(F, \tau(d))}\right) .
$$


If $v<\iota(d)$, that is, $g(E, v)<g(E, \iota(d))$, then the term $E_{g(E, v)}\left(\vec{\delta}_{g(E, v)}+\vec{\delta}_{g(E, \iota(d))}\right)$ represents a collapsible 1-cell and so disappears in the formula.

Given two deleted edges $d$ and $d^{\prime}$, there are three possible relative positions of them as classified in the lemma. In case $(1)$, since $\iota(d)<\iota\left(d^{\prime}\right)$,

$$
\widetilde{\partial}\left(d \cup d^{\prime}\right)=\widetilde{R}\left(d \cup \iota\left(d^{\prime}\right)\right)-\widetilde{R}\left(d \cup \tau\left(d^{\prime}\right)\right)-\widetilde{R}\left(d^{\prime} \cup \iota(d)\right)+\widetilde{R}\left(d^{\prime} \cup \tau(d)\right) .
$$

Since $\tau(d)<\tau\left(d^{\prime}\right)<\iota\left(d^{\prime}\right), \widetilde{R}\left(d^{\prime} \cup \tau(d)\right)=d^{\prime}$, and since $\iota(d)<\tau\left(d^{\prime}\right)<\iota\left(d^{\prime}\right)$, $\widetilde{R}\left(d^{\prime} \cup \iota(d)\right)=d^{\prime}$. So we obtain the desired formula. Similarly, we can obtain the other two formulae.

Next we consider the third boundary homomorphisms in Morse complexes that are needed to prove that braid groups of planar graphs containing $S_{0}$ are not rightangled Artin groups. Let $\Gamma^{\prime}$ be either $S_{0}$ or $\Theta$. Choose a maximal tree $T^{\prime}$ of $\Gamma^{\prime}$ and its planar embedding as in Figure 16] so that there are no $i$-cells in $U D_{n} \Gamma^{\prime}$ for $i \geq 3$. As explained in \$2.2, a planar embedding of $T^{\prime}$ and a choice of a vertex as a base vertex determine an order on vertices of $\Gamma^{\prime}$. Now suppose there is an embedding $i: \Gamma^{\prime} \rightarrow \Gamma$ into another planar graph $\Gamma$. We may choose a maximal tree $T$ of $\Gamma$ so that $\left.i\right|_{T^{\prime}}: T^{\prime} \rightarrow T$ is an embedding. Then the image of the base vertex $T^{\prime}$ naturally becomes the base vertex of $T$, and we may extend the planar embedding of $T^{\prime}$ over $T$. The extended planar embedding of $T$, together with the choice of base vertex, determine an order on vertices of $\Gamma$. Then it is easy to see that the embedding $i$ preserves the base vertex, the order, and the deleted edges. Thus the embedding $i$ naturally induces the chain map $\tilde{i}$ between two Morse chain complexes, and so it induces homomorphisms $i_{*}: H_{*}\left(B_{n} \Gamma^{\prime}\right) \rightarrow H_{*}\left(B_{n} \Gamma\right)$. Note that the requirement of an embedding $i: \Gamma^{\prime} \rightarrow \Gamma$ is simpler than that in $\$ 3.3$ because the braid indices for $\Gamma^{\prime}$ and $\Gamma$ are the same in this section.

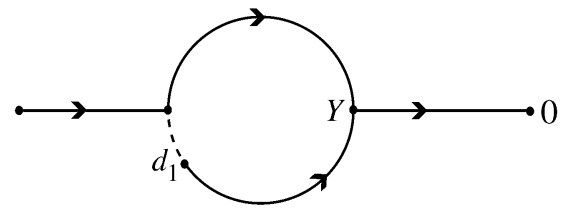

(a) $S_{0}$

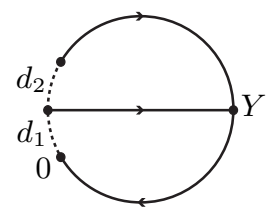

(b) $\Theta$

FiguRE 16. Maximal trees and orders of $S_{0}$ and $\Theta$

It is difficult to give a general formula for the boundary homomorphism $\widetilde{\partial}$ : $M_{3}\left(U D_{n} \Gamma\right) \rightarrow M_{2}\left(U D_{n} \Gamma\right)$. Fortunately, all we will need in $\$ 4.3$ is the relationship between $\widetilde{\partial}\left(M_{3}\left(U D_{n} \Gamma\right)\right)$ and $\widetilde{i}\left(M_{2}\left(U D_{n} \Gamma^{\prime}\right)\right)$. Using the notation in Figure 16, every critical 2-cell in $U D_{n} S_{0}$ must contain $Y_{2}(a, b) \cup d_{1}$ as a subset and every critical 2-cell in $U D_{n} \Theta$ is the form $Y_{2}(a, b) \cup d_{j}$ for $j=1,2$. It turns out that we need not consider critical 2-cells containing $d_{2}$ for $U D_{n} \Theta$. Let $c$ be a critical 2-cell containing $d_{1}$ in $U D_{n} \Gamma^{\prime}$. Then $\tilde{i}(c)$ must contain $X_{s}\left(a \vec{\delta}_{r}+b \vec{\delta}_{s}\right) \cup q$ as a subset for $1 \leq r<s \leq \mu$, where $i(Y)=X, i\left(d_{1}\right)=q$, and $\mu+1$ is the valency of $X$ in the maximal tree $T$ of $\Gamma$. Consider the subgroup $M_{q}$ of $M_{2}\left(U D_{n} \Gamma\right)$ generated by all critical 2-cells containing the deleted edge $q$ of $\Gamma$ that is the image of the deleted edge $d_{1}$ of $\Gamma^{\prime}$ under the embedding $i$. In particular, $\widetilde{i}\left(M_{2}\left(U D_{n} \Gamma^{\prime}\right)\right) \subset M_{q}$ if $\Gamma^{\prime}=S_{0}$. For the projection $\pi: M_{2}\left(U D_{n} \Gamma\right) \rightarrow M_{q}$, the composites $\pi \circ \widetilde{\partial}$ and $\pi \circ \widetilde{R}$ will be denoted by $\widetilde{\partial}_{q}$ and 
$\widetilde{R}_{q}$. From now on, a vertex denoted by the letter $X$ and a deleted edge denoted by the letter $q$ will be reserved as above when we consider a graph $\Gamma$ containing $S_{0}$ or $\Theta$.

Let $c$ be a critical 3-cell in $U D_{n} \Gamma$ containing $q$. When we compute $\widetilde{\partial}_{q}(c)$, two faces of $c$ determined by taking end vertices of $q$ do not contain $q$, and so we only need to consider the remaining four faces of $\partial(c)$. Consequently, any vertex blocked by $q$ plays no role in computing $\widetilde{\partial}_{q}(c)=\widetilde{R}_{q} \partial(c)$. Thus we immediately obtain the following modified version of Lemma 4.6,

Lemma 4.9. Suppose a redundant cell $c$ has an unblocked vertex $v$ and $e$ is the edge starting from $v$. Assume also that if a vertex $w$ that is either in $c$ or an end vertex of an edge other than $q$ in $c$ satisfies $\tau(e)<w<\iota(e)$, then $w$ is blocked by q. Then $\widetilde{R}_{q}(c)=\widetilde{R}_{q} V_{e}(c)$.

Let $v$ be a vertex in $\Gamma$ with the maximal tree $T$ and $e$ be an edge in $\Gamma$. The edge $e$ is said to be separated by $v$ if $\iota(e)$ and $\tau(e)$ lie in two distinct components of $T-\{v\}$. It is clear that only a deleted edge can be separated by a vertex. If a deleted edge $d$ is not separated by $v$, then $\iota(d), \tau(d)$, and $\iota(d) \wedge \tau(d)$ are all in the same component of $T-\{v\}$. We only need to compute images of redundant 2-cells under $\widetilde{R}_{q}$, and we can strengthen Lemma 4.9 for redundant 2-cells.

Lemma 4.10. Let c be a redundant 2-cell containing the deleted edge $q$ and another edge $p$. Suppose the redundant 2-cell $c$ has an unblocked vertex $v$ and $e$ is the edge starting from $v$ satisfying the following conditions:

(a) Every vertex $w$ in $c$ satisfying $\tau(e)<w<\iota(e)$ is blocked.

(b) If an end vertex $w$ of $p$ satisfies $\tau(e)<w<\iota(e)$, then $p$ is not separated by any of $\tau(e)$ and end vertices of $q$.

Then $\widetilde{R}_{q}(c)=\widetilde{R}_{q} V_{e}(c)$. Therefore if $p$ is not a deleted edge, then $\widetilde{R}_{q}(c)=\widetilde{R}_{q} \widetilde{V}(c)$.

Proof. If both end vertices of $p$ are not between $\tau(e)$ and $\iota(e)$, then $c$ satisfies the hypothesis of Lemma 4.9. So we are done. Otherwise $p$ is not separated by any of $\tau(e)$ and two end vertices of $q$. So $\iota(p)$ and $\tau(p)$ are in the same component $T_{p}$ of $T-\{\tau(e), \iota(q), \tau(q)\}$. Let $w$ be any vertex in $T_{p}$. Let $c_{w}$ denote a cell obtained from $c$ replacing $v$ and $p$ by $e$ and $w$. Let $c^{\prime}$ be a redundant 2-cell obtained from $c_{\iota(p)}$ replacing vertices in $T_{p}$ to other vertices that are all in $T_{p}$. Note that $\left\{c^{\prime}\right\}$ is a finite set since the number of vertices in $T_{p}$ is finite. If $c^{\prime}$ has no unblocked vertex in $T_{p}$, then $c^{\prime}$ is unique because $\Gamma$ is sufficiently subdivided. This 2-cell is denoted by $c_{p}$. If $c^{\prime}$ has an unblocked vertex in $T_{p}$, then $c^{\prime}$ has the smallest unblocked vertex $u$ in $T_{p}$ that satisfies the hypothesis of Lemma 4.9 since $c^{\prime}$ contains exactly two edges $q$ and $e$ and every vertex in $T_{p}$ is between $\tau(e)$ and $\iota(e)$. So $\widetilde{R}_{q}\left(c^{\prime}\right)=\widetilde{R}_{q} V_{e^{\prime}}\left(c^{\prime}\right)$, where $e^{\prime}$ is the edge starting from $u$. By repeating this argument, we have $\widetilde{R}_{q}\left(c_{\iota(p)}\right)=$ $\widetilde{R}_{q}\left(c_{p}\right)=\widetilde{R}_{q}\left(c_{\tau(p)}\right)$ because $V_{e^{\prime}}\left(c^{\prime}\right)$ is also in $\left\{c^{\prime}\right\}$ that is a finite set. Thus

$$
\widetilde{R}_{q}(c)=\widetilde{R}_{q} V_{e}(c) \pm\left\{\widetilde{R}_{q}\left(c_{\iota(p)}\right)-\widetilde{R}_{q}\left(c_{\tau(p)}\right)\right\}=\widetilde{R}_{q} V_{e}(c),
$$

where the sign \pm is determined by the order between the initial vertices of three edges $p, q$ and $e$. Therefore if $p$ is not a deleted edge, then the smallest unblocked vertex in $c$ satisfies the hypothesis of this lemma. So $\widetilde{R}_{q}(c)=\widetilde{R}_{q} V(c)$. By repeating this argument, we have $\widetilde{R}_{q}(c)=\widetilde{R}_{q} \widetilde{V}(c)$.

To demonstrate how to compute an image of $\widetilde{\partial}_{q}$, we first give an example. 


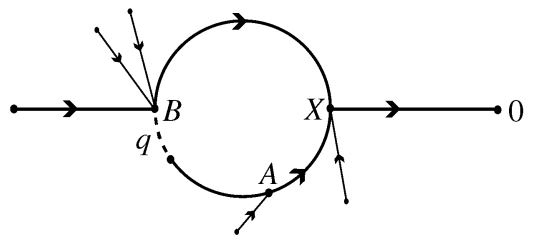

Figure 17. Graph containing $S_{0}$ but not $\Theta$

Example 4.11. For a graph $\Gamma$ and its maximal tree and the order given in Figure 17, we compute $\widetilde{\partial}_{q}(c)$ for the critical 3-cell $c=X_{3}(1,2,2) \cup q \cup A_{2}(1,1) \cup B(2,0,0)$ in $M_{3}\left(U D_{n} \Gamma\right)$.

Since $\iota\left(A_{2}(0,1)\right)<\iota\left(X_{3}(0,0,1)\right)<\iota(q)$, we have

$$
\begin{aligned}
\partial_{q}(c)= & +\left(X(1,2,2) \cup q \cup A_{2}(1,1) \cup B(2,0,0)\right) \\
& -\left(\dot{X}(1,2,1) \cup q \cup A_{2}(1,1) \cup B(2,0,0)\right) \\
& -\left(X_{3}(1,2,2) \cup q \cup A(1,1) \cup B(2,0,0)\right) \\
& +\left(X_{3}(1,2,2) \cup q \cup \dot{A}(1,0) \cup B(2,0,0)\right) .
\end{aligned}
$$

Consider the pair of faces determined by the edge $A_{2}(0,1)$. The edge $X_{3}(0,0,1)$ is not a deleted edge, and their images under $\widetilde{V}$ are equal, so their images $\widetilde{R}_{q}$ are also equal by Lemma 4.10. Thus the images cancel out in $\widetilde{\partial}_{q}(c)$ because they have opposite signs. Similarly, the images of the pair of faces determined by the edge $X_{3}(0,0,1)$ under $\widetilde{R}_{q}$ also cancel out. Thus

$$
\widetilde{\partial}_{q}\left(X_{3}(1,2,2) \cup q \cup A_{2}(1,1) \cup B(2,0,0)\right)=0 .
$$

Lemma 4.12. Let $\Gamma$ be a finite planar graph containing $S_{0}$ or $\Theta$. Suppose that $c$ is a critical 3-cell in $U D_{n} \Gamma$ as the form $A_{k}(\vec{a}) \cup B_{\ell}(\vec{b}) \cup q \cup C(\vec{c})$ where $C=\iota(q)$. Then $\widetilde{\partial}_{q}(c)=0$.

Proof. We consider two faces of $\partial(c)$ determined by $A_{k}\left(\vec{\delta}_{k}\right)$. They contain $B_{\ell}\left(\vec{\delta}_{\ell}\right)$ that is not a deleted edge and have the same image under $\widetilde{V}$. By Lemma 4.10, their images under $\widetilde{R}_{q}$ are the same. Since they have opposite signs, they cancel out each other in $\widetilde{\partial}_{q}(c)$, by the same reason images of two faces determined by $B_{\ell}\left(\vec{\delta}_{\ell}\right)$ under $\widetilde{R}_{q}$ cancel out each other in $\widetilde{\partial}_{q}(c)$. Consequently, $\widetilde{\partial}_{q}(c)=0$.

If a critical 3-cell $c$ contains other deleted edges in addition to $q$, we have to deal with too many cases to compute $\widetilde{\partial}_{q}(c)$. In order to narrow down the possibilities, we need the following lemma.

Lemma 4.13. Let $\Gamma^{\prime}$ be either $S_{0}$ or $\Theta$ embedded in a plane as in Figure 16, Let $\Gamma$ be a finite planar graph containing $\Gamma^{\prime}$. Assume that if $\Gamma^{\prime}$ is $S_{0}$, then $\Gamma$ does not contain $\Theta$. Then we can choose an embedding $i: \Gamma^{\prime} \rightarrow \Gamma$ and modify the planar embedding of $\Gamma$ so that all edges of $\Gamma$ that are incident to the vertex $X=i(Y)$ and are not in the image of $i$ lie between $i\left(e_{0}\right)$ and $i\left(e_{1}\right)$, where $e_{0}, e_{1}$ are edges incident to $Y$ in $\Gamma^{\prime}$ such that $e_{0}$ heads for the base vertex 0 and $e_{1}$ is next to $e_{0}$ clockwise.

Proof. Let $e_{2}$ denote the remaining edge incident to $Y$ in $\Gamma^{\prime}$. Figure 18 or Figure 19 show only the part of $\Gamma$ consisting of $i\left(\Gamma^{\prime}\right)$ and edges incident to $X$. Suppose that 
$\Gamma$ contains $S_{0}$ but not $\Theta$. Then we choose an embedding $i: S_{0} \rightarrow \Gamma$ such that $\Gamma$ has no edges incident to $X$ between $i\left(e_{2}\right)$ and $i\left(e_{0}\right)$. Since $\Gamma$ contains no $\Theta$, every edge incident to $X$ in $\Gamma$ between $i\left(e_{1}\right)$ and $i\left(e_{2}\right)$ should not be joined to $i\left(\Gamma^{\prime}\right)$ by an edge path (indicated by a dotted arc in Figure 18) unless it is a loop at $X$. Thus the subgraph attached to these edges is free from $i\left(\Gamma^{\prime}\right)$ except at $X$, and it can be flipped and placed between $i\left(e_{0}\right)$ and $i\left(e_{1}\right)$ by modifying the planar embedding of $\Gamma$.

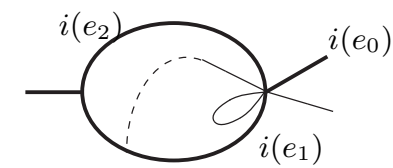

(a) Before modification

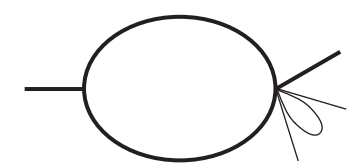

(b) After modification

FiguRE 18. $i: S_{0} \rightarrow \Gamma$

Suppose that $\Gamma$ contains $\Theta$. Then we choose an embedding $i: \Theta \rightarrow \Gamma$ such that (i) $i(\Theta)$ is outmost in the sense that it is engulfed by no other larger embedding $i^{\prime}$ of $\Theta$ such that $i^{\prime}(Y)=X$, and (ii) no other edge path joining $X$ and another vertex on $i\left(e_{1} \cup e_{2}\right)$ lies inside the circle $i\left(e_{1} \cup e_{2}\right)$. By (i), every edge incident to $X$ in $\Gamma$ between $i\left(e_{2}\right)$ and $i\left(e_{0}\right)$ should not be joined to $i\left(\Gamma^{\prime}\right)$ by an edge path (indicated by a dotted arc in Figure 19) unless it is a loop at $X$. By (ii), every edge incident to $X$ in $\Gamma$ between $i\left(e_{1}\right)$ and $i\left(e_{2}\right)$ should not be joined to $i\left(\Gamma^{\prime}\right)$ by an edge path unless it is a loop at $X$. Again the subgraphs attached to these edges are free from $i\left(\Gamma^{\prime}\right)$ except at $X$, and they can be placed between $i\left(e_{0}\right)$ and $i\left(e_{1}\right)$ by modifying the planar embedding of $\Gamma$.

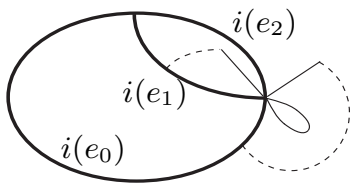

(a) Before modification

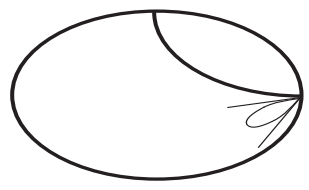

(b) After modification

Figure 19. $i: \Theta \rightarrow \Gamma$

After we choose an embedding $i: \Gamma^{\prime} \rightarrow \Gamma$ and a planar embedding of $\Gamma$ given by Lemma 4.13, we choose a maximal tree $T$ of $\Gamma$ so that it satisfies the following:

(1) Delete the edge that is the image of $d_{1}$ or $d_{2}$ in $S_{0}$ or $\Theta$ under $i$.

(2) Delete an edge incident to $\iota(q)$ whenever we need to break a circuit containing $\iota(q)$.

(3) Delete an edge incident to $X$ whenever we need to break a circuit containing $X$ but not $\iota(q)$ and $\Gamma$ does not contain $\Theta$.

(4) All other deleted edges have valency 2 at both ends. 
The conditions on a maximal tree $T$ of a planar graph $\Gamma$, together with an embedding $i: \Gamma^{\prime} \rightarrow \Gamma$ given by Lemma 4.13, will be referred to as $\operatorname{Cond}(\Gamma, i, T)$. There are useful consequences in assuming $\operatorname{Cond}(\Gamma, i, T)$. Condition (1) guarantees that $i$ induces a chain map between Morse complexes if $i$ preserves the order of $\Gamma^{\prime}$. Condition (2) guarantees that there are no deleted edges separated by $\iota(q)$. Condition (3) implies that if $X$ separates a deleted edge in a graph containing $S_{0}$ but not $\Theta$, then the deleted edge is $q$. There are no vertices blocked by deleted edges satisfying condition (4).

After fixing the base vertex of $\Gamma$ by choosing a vertex of valency 1 in $T$ that can be joined to $i(0)$ via an edge path without $i\left(e_{0}\right)$, we give $\Gamma$ the order determined by the planar embedding of $T$. Then the embedding $i: \Gamma^{\prime} \rightarrow \Gamma$ is order preserving, that is, $v_{1}<v_{2}$ implies $i\left(v_{1}\right)<i\left(v_{2}\right)$ for vertices $v_{1}, v_{2}$ of $\Gamma^{\prime}$. Since $\operatorname{Cond}(\Gamma, i, T)$ assumes the conclusion of Lemma 4.13, it implies

$$
\tilde{i}\left(Y_{2}(a, b) \cup d_{1}\right)=X_{s}\left(a \vec{\delta}_{\mu-1}+b \vec{\delta}_{\mu}\right) \cup q,
$$

where $\mu+1$ is the valency of $X$ in a maximal tree $T$ of $\Gamma$. The amount of computation of $\partial_{q}$ will be considerably reduced by assuming $\operatorname{Cond}(\Gamma, i, T)$ since it limits a great deal of possibilities.

In $\$ 4.3$, we will need the fact that $\tilde{i}\left(M_{2}\left(U D_{n} \Gamma^{\prime}\right)\right) \cap \tilde{\partial}\left(M_{3}\left(U D_{n} \Gamma\right)\right)=\{0\}$. Under the assumption Cond $(\Gamma, i, T), \tilde{i}\left(M_{2}\left(U D_{n} \Gamma^{\prime}\right)\right)$ is generated by critical 2-cells of the form $X_{\mu}(\vec{x}) \cup q$, where the vertex $X$ is of valency $\mu+1$ in $T$. To demonstrate how to compute $\widetilde{\partial}_{q}(c)$, let us first see an example.

Example 4.14. For a graph $\Gamma$ and its maximal tree and the order given in Figure 20, we compute $\widetilde{\partial}_{q}(c)$ for a 3 -cell $X_{2}(2,1) \cup q \cup d$ in $M_{3}\left(U D_{n} \Gamma\right)$.

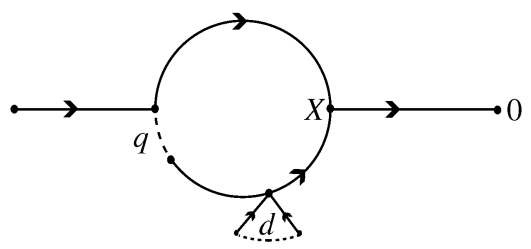

FiguRE 20. Graph containing $X_{2}(2,1) \cup q \cup d$

Since $\iota(d)<\iota\left(X_{2}(0,1)\right)<\iota(q)$, we have

$$
\begin{aligned}
\widetilde{\partial}_{q}(c)= & \widetilde{R}_{q}(q \cup d \cup X(2,1))-\widetilde{R}_{q}(q \cup d \cup \dot{X}(2,0)) \\
& -\widetilde{R}_{q}\left(X_{2}(2,1) \cup q \cup \iota(d)\right)+\widetilde{R}_{q}\left(X_{2}(2,1) \cup q \cup \tau(d)\right) .
\end{aligned}
$$

After the above four faces are rewritten to eliminate all unblocked vertices satisfying the hypothesis of Lemma 4.10, we have

$$
\begin{aligned}
& \widetilde{R}_{q}(q \cup d \cup X(2,1))=\widetilde{R}_{q}(q \cup d \cup \dot{X}(2,0))=q \cup d, \\
& \widetilde{R}_{q}\left(X_{2}(2,1) \cup q \cup \iota(d)\right)=\widetilde{R}_{q}\left(X_{2}(2,1) \cup q \cup \tau(d)\right)=X_{2}(3,1) \cup q .
\end{aligned}
$$

Consequently, we have $\widetilde{\partial}_{q}(c)=0$. 
Lemma 4.15. Let $\Gamma$ be a planar graph satisfying $\operatorname{Cond}(\Gamma, i, T)$. Suppose that $c$ is a critical 3-cell in $U D_{n} \Gamma$ of the form $A_{k}(\vec{a}) \cup q \cup d$. If c satisfies one of the following:

(i) $A \neq X$,

(ii) $A=X$ and $k<\mu$,

(iii) $A=X, k=\mu$ and $d$ is not separated by $X$,

then $\widetilde{\partial}(c)$ contains no critical 2 -cells of the form $X_{\mu}(\vec{x}) \cup q$ as a summand.

Proof. First consider two faces of $\partial_{q}(c)$ determined by $d$, that is, $A_{k}(\vec{a}) \cup q \cup\{v\}$, where $v$ is either $\iota(d)$ or $\tau(d)$. Since $A_{k}\left(\vec{\delta}_{k}\right)$ is not a deleted edge,

$$
\widetilde{R}_{q}\left(A_{k}(\vec{a}) \cup q \cup\{v\}\right)=\widetilde{R}_{q} \widetilde{V}\left(A_{k}(\vec{a}) \cup q \cup\{v\}\right)
$$

by Lemma 4.10. If $A \neq X$ or $k \neq \mu$, then $\widetilde{R}_{q} \widetilde{V}\left(A_{k}(\vec{a}) \cup q \cup\{v\}\right)$ cannot be a critical 2-cell of the form $X_{\mu}(\vec{x}) \cup q$. If $A=X$ and $k=\mu$, then their images under $\widetilde{R}_{q} \widetilde{V}$ are equal since $d$ is not separated by $X$. So the two faces cancel out each other in $\widetilde{\partial}_{q}(c)$.

Now consider two faces determined by $A_{k}\left(\vec{\delta}_{k}\right)$ under $\widetilde{R}_{q}$. If $A \neq X$, then $A$ and $A\left(\vec{\delta}_{k}\right)$ are in the same branch of $X$. If there is a critical 2-cell of the form $X_{\mu}(\vec{x}) \cup q$ in the image of a face under $\widetilde{R}_{q}$, then the critical 2-cell is also in the image of the other face under $\widetilde{R}_{q}$. Thus all critical 2-cells of the form $X_{\mu}(\vec{x}) \cup q$ cancel out in $\widetilde{\partial}_{q}(c)$ since each pair of faces has the opposite sign in $\partial_{q}(c)$. If $A=X$ and $k<\mu$, then all critical 2-cells of the form $X_{\mu}(\vec{x}) \cup q$ also cancel out in $\widetilde{\partial}_{q}(c)$ because the numbers of vertices in each pair of faces on the $\mu$-th branch of $X$ are equal. Finally, if $A=X$ and $k=\mu$, then there are no critical 2-cells of the form $X_{\mu}(\vec{x}) \cup q$ in the image of faces under $\widetilde{R}_{q}$ since $d$ is not separated by $X$.

If $\Gamma$ does not contain $\Theta$, then there are blocked vertices by a deleted edge $d_{X}$ incident to $X$. So we must consider a critical 3-cell in $U D_{n} \Gamma$ of the form $A_{k}(\vec{a}) \cup$ $q \cup d_{X} \cup X(\vec{x})$. Any vertex blocked by $X$ plays no role in the proof of the above lemma. Thus we have the following corollary.

Corollary 4.16. Let $\Gamma$ be a graph that does not contain $\Theta$ and satisfies $\operatorname{Cond}(\Gamma, i, T)$. Suppose that $c$ is a critical 3-cell in $U D_{n} \Gamma$ of the form $A_{k}(\vec{a}) \cup q \cup d_{X} \cup X(\vec{x})$. Then $\widetilde{\partial}(c)$ contains no critical 2-cells of the form $X_{\mu}(\vec{x}) \cup q$ as a summand.

Now consider a critical 3-cell containing $X_{\mu}(\vec{x})$ and a deleted edge $d$ such that $X$ separates $d$.

Example 4.17. Let $\Gamma$ be a graph as in Figure 21, We compute $\widetilde{\partial}_{q}(c)$ for the 3 -cell $c=X_{2}(1,2) \cup q \cup d$ in $M_{3}\left(U D_{n} \Gamma\right)$. Note that $A=X \wedge \tau(d)$.

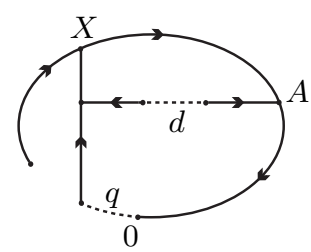

FiguRE 21. Graph containing $X_{2}(1,2) \cup q \cup d$ 
Since $\iota(d)<\iota(q)<\iota\left(X_{2}(0,1)\right)$, using Lemma 4.9 we have

$$
\begin{aligned}
\widetilde{\partial}_{q}(c)= & -\widetilde{R}_{q}(q \cup d \cup X(1,2))+\widetilde{R}_{q}(q \cup d \cup \dot{X}(1,1)) \\
& -\widetilde{R}_{q}\left(X_{2}(1,2) \cup q \cup \iota(d)\right)+\widetilde{R}_{q}\left(X_{2}(1,2) \cup q \cup \tau(d)\right) \\
= & -\widetilde{R}_{q}(q \cup d \cup X(0,2) \cup A(0,1))+\widetilde{R}_{q}(q \cup d \cup X(0,1) \cup A(0,2)) \\
& -\left(X_{2}(2,2) \cup q\right)+\left(X_{2}(1,2) \cup q\right) .
\end{aligned}
$$

Since $q \cup d \cup X(0,2) \cup A(0,1)$ has the negative sign in the boundary of the collapsible 3-cell $A_{2}(0,1) \cup q \cup d \cup X(0,2)$,

$$
\begin{aligned}
& \widetilde{R}_{q}(q \cup d \cup X(0,2) \cup A(0,1)) \\
&=\widetilde{R}_{q}\left(\partial\left(A_{2}(0,1) \cup q \cup d \cup X(0,2)\right)+q \cup d \cup X(0,2) \cup A(0,1)\right) \\
&=\widetilde{R}_{q}(q \cup d \cup\{A\} \cup X(0,2))+\widetilde{R}_{q}\left(A_{2}(0,1) \cup q \cup \iota(d) \cup X(0,2)\right) \\
&-\widetilde{R}_{q}\left(A_{2}(0,1) \cup q \cup \tau(d) \cup X(0,2)\right) \\
&= \widetilde{R}_{q}(q \cup d \cup X(0,2))-\left(A_{2}(1,3) \cup q\right) .
\end{aligned}
$$

By repeating a similar argument,

$$
\begin{aligned}
\widetilde{R}_{q}(q \cup d \cup X(0,2))= & \widetilde{R}_{q}\left(\partial\left(X_{2}(0,2) \cup q \cup d\right)+q \cup d \cup X(0,2)\right) \\
= & \widetilde{R}_{q}(q \cup d \cup \dot{X}(0,1))-\widetilde{R}_{q}\left(X_{2}(0,2) \cup q \cup \iota(d)\right) \\
& +\widetilde{R}_{q}\left(X_{2}(0,2) \cup q \cup \tau(d)\right) \\
= & \widetilde{R}_{q}(q \cup d \cup X(0,1))-\left(A_{2}(1,2) \cup q\right)-\left(X_{2}(1,2) \cup q\right)
\end{aligned}
$$

and $\widetilde{R}_{q}(q \cup d \cup \dot{X}(1,1))=\widetilde{R}_{q}(q \cup d \cup X(0,1))-\left(A_{2}(1,3) \cup q\right)-\left(A_{2}(1,2) \cup q\right)$. Consequently, we have $\widetilde{\partial}_{q}(c)=2\left(X_{2}(1,2) \cup q\right)-\left(X_{2}(2,2) \cup q\right)$.

A graph considered in the following lemma necessarily contains $\Theta$ since it satisfies Cond $(\Gamma, i, T)$ and has a deleted edge other than $q$ separated by $X$.

Lemma 4.18. Let $\Gamma$ be a planar graph satisfying $\operatorname{Cond}(\Gamma, i, T)$. Suppose that $c$ is a critical 3-cell in $U D_{n} \Gamma$ of the form $X_{\mu}(\vec{x}) \cup q \cup d$ and $d$ is separated by the vertex $X$ but not by $X\left(\vec{\delta}_{\mu}\right)$. Then

$$
\begin{aligned}
\widetilde{\partial}_{q}(c)= & \left(X_{\mu}\left(\vec{x}+\vec{\delta}_{g(X, \tau(d))}\right) \cup q\right)-\left(X_{\mu}\left(\vec{x}+\vec{\delta}_{g(X, \iota(d))}\right) \cup q\right) \\
& -\left(X_{\mu}\left(\vec{\delta}_{g(X, \tau(d))}+x_{\mu} \vec{\delta}_{\mu}\right) \cup q\right)+\left(X_{\mu}\left(\vec{\delta}_{g(X, \iota(d))}+x_{\mu} \vec{\delta}_{\mu}\right) \cup q\right)+\text { Irrelevant },
\end{aligned}
$$

where the part "Irrelevant" contains no critical 2-cells of the form $X_{\mu}(\vec{y}) \cup q$, and $x_{\mu}$ is the $\mu$-th component of $\vec{x}$. The expression may contain collapsible 2 -cells that should be regarded as trivial

Proof. Since $d$ is separated by $X$ but not by $\iota(q)$ due to $\operatorname{Cond}(\Gamma, i, T), X$ and both ends of $d$ are in the same component of $T-\{\iota(q)\}$. Since $d$ is not separated by $X\left(\vec{\delta}_{\mu}\right), \iota(d)<X\left(\vec{\delta}_{\mu}\right)$. Also, $\iota(d)<\iota(q)$ by $\operatorname{Cond}(\Gamma, i, T)$. Thus we only need to consider the two possibilities $\iota(d)<X\left(\vec{\delta}_{\mu}\right)<\iota(q)$ and $\iota(d)<\iota(q)<X\left(\vec{\delta}_{\mu}\right)$. Assume $\iota(d)<X\left(\vec{\delta}_{\mu}\right)<\iota(q)$. Then we have

$$
\begin{aligned}
\widetilde{\partial}_{q}(c)= & \widetilde{R}_{q}\left(X_{\mu}(\vec{x}) \cup q \cup \tau(d)\right)-\widetilde{R}_{q}\left(X_{\mu}(\vec{x}) \cup q \cup \iota(d)\right) \\
& -\widetilde{R}_{q}\left(q \cup d \cup \dot{X}\left(\vec{x}-\vec{\delta}_{\mu}\right)\right)+\widetilde{R}_{q}(q \cup d \cup X(\vec{x})) .
\end{aligned}
$$


For the pair of faces of $c$ determined by $d$, we can apply Lemma 4.10 since $X_{\mu}\left(\vec{\delta}_{\mu}\right)$ is not a deleted edge. Then we have

$$
\begin{aligned}
\widetilde{R}_{q}\left(X_{\mu}(\vec{x}) \cup q \cup \tau(d)\right)-\widetilde{R}_{q}\left(X_{\mu}(\vec{x}) \cup q \cup \iota(d)\right) \\
\quad=X_{\mu}\left(\vec{x}+\vec{\delta}_{g(X, \tau(d))}\right) \cup q-X_{\mu}\left(\vec{x}+\vec{\delta}_{g(X, \iota(d))}\right) \cup q .
\end{aligned}
$$

To consider the pair of faces determined by $X_{\mu}\left(\vec{\delta}_{\mu}\right)$, let $A=X \wedge \tau(d)$. Since $d$ is separated by $X, X \wedge \iota(d)=X$. Since the smallest unblocked vertex of $q \cup d \cup \dot{X}\left(\vec{x}-\vec{\delta}_{\mu}\right)$ is $X$, it does not give a critical 2-cell of the form $X_{\mu}\left(\overrightarrow{x^{\prime}}\right) \cup q$ in its image under $\widetilde{R}_{q}$ as follows:

$\widetilde{R}_{q}\left(q \cup d \cup \dot{X}\left(\vec{x}-\vec{\delta}_{\mu}\right)\right)=\widetilde{R}_{q}\left(q \cup d \cup X\left(\vec{x}-\vec{\delta}_{\mu}\right)\right)-A_{g(A, X)}\left(|\vec{x}| \vec{\delta}_{g(A, X)}+\vec{\delta}_{g(A, \tau(d))}\right) \cup q$,

where the second term disappears if $A=X$. On the other hand, if the smallest unblocked vertex $v$ of $q \cup d \cup X(\vec{x})$ satisfies $g(X, v) \neq \mu$, it does not produce a critical 2-cell of the form $X_{\mu}\left(\overrightarrow{x^{\prime}}\right) \cup q$ since $v<X\left(\vec{\delta}_{\mu}\right)$, that is, we have

$$
\widetilde{R}_{q}(q \cup d \cup X(\vec{x}))=\widetilde{R}_{q}(q \cup d \cup X(\vec{x}-1))+\text { Irrelevant terms. }
$$

By iterating this step, we have

$$
\widetilde{R}_{q}(q \cup d \cup X(\vec{x}))=\widetilde{R}_{q}\left(q \cup d \cup X\left(x_{\mu} \vec{\delta}_{\mu}\right)\right)+\text { Irrelevant terms. }
$$

From $\iota(d)<X\left(\vec{\delta}_{\mu}\right)<\iota(q)$, we have

$$
\begin{aligned}
\widetilde{R}_{q}\left(q \cup d \cup X\left(x_{\mu} \vec{\delta}_{\mu}\right)\right)= & \widetilde{R}_{q}\left(-\partial\left(q \cup d \cup X_{\mu}\left(x_{\mu} \vec{\delta}_{\mu}\right)\right)+q \cup d \cup X\left(x_{\mu} \vec{\delta}_{\mu}\right)\right) \\
= & \widetilde{R}_{q}\left(q \cup d \cup X\left(\left(x_{\mu}-1\right) \vec{\delta}_{\mu}\right)\right)+\widetilde{R}_{q}\left(X_{\mu}\left(x_{\mu} \vec{\delta}_{\mu}\right) \cup q \cup \iota(d)\right) \\
& -\widetilde{R}_{q}\left(X_{\mu}\left(x_{\mu} \vec{\delta}_{\mu}\right) \cup q \cup \tau(d)\right) \\
= & \widetilde{R}_{q}\left(q \cup d \cup X\left(\left(x_{\mu}-1\right) \vec{\delta}_{\mu}\right)\right. \\
& -A_{g(A, X)}\left(x_{\mu} \vec{\delta}_{g(A, X)}+\vec{\delta}_{g(A, \tau(d))}\right) \cup q \\
& +X_{\mu}\left(x_{\mu} \vec{\delta}_{\mu}+\vec{\delta}_{g(X, \iota(d))}\right) \cup q-X_{\mu}\left(x_{\mu} \vec{\delta}_{\mu}+\vec{\delta}_{g(X, \tau(d))}\right) \cup q .
\end{aligned}
$$

So we obtain the desired formula for the case $\iota(d)<X\left(\vec{\delta}_{\mu}\right)<\iota(q)$. Similarly, we can proceed in the case $\iota(d)<\iota(q)<X\left(\vec{\delta}_{\mu}\right)$.

Example 4.19. Let $\Gamma$ be a graph as in Figure 22, and we choose a maximal tree and give an order as in Figure 22. We compute $\widetilde{\partial}_{q}(c)$ for the 3-cell $q \cup d \cup d^{\prime}$ in $M_{3}\left(U D_{n} \Gamma\right)$.

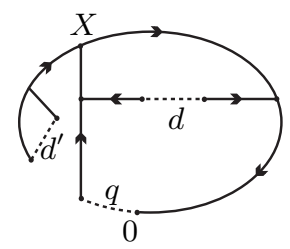

FiguRE 22. Graph containing $q \cup d \cup d^{\prime}$ 
Since $\iota(d)<\iota(q)<\iota\left(d^{\prime}\right)$, using Lemma 4.10 we have

$$
\begin{aligned}
\widetilde{\partial}_{q}(c)= & -\widetilde{R}_{q}\left(q \cup d^{\prime} \cup \iota(d)\right)+\widetilde{R}_{q}\left(q \cup d^{\prime} \cup \tau(d)\right) \\
& -\widetilde{R}_{q}\left(q \cup d \cup \iota\left(d^{\prime}\right)\right)+\widetilde{R}_{q}\left(q \cup d \cup \tau\left(d^{\prime}\right)\right) \\
= & -\left(q \cup d^{\prime}\right)+\left(q \cup d^{\prime}\right)-\widetilde{R}_{q}\left(q \cup d \cup\left\{X\left(\vec{\delta}_{\mu}\right)\right\}\right)+\widetilde{R}_{q}\left(q \cup d \cup\left\{X\left(\vec{\delta}_{\mu}\right)\right\}\right)=0 .
\end{aligned}
$$

Lemma 4.20. Let $\Gamma$ be a planar graph satisfying $\operatorname{Cond}(\Gamma, i, T)$. Suppose that $c$ is a critical 3-cell in $U D_{n} \Gamma$ of the form $q \cup d \cup d^{\prime}$. If both $d$ and $d^{\prime}$ are not separated by $X\left(\vec{\delta}_{\mu}\right)$, then $\widetilde{\partial}(c)$ contains no critical 2-cells of the form $X_{\mu}(\vec{x}) \cup q$ as a summand.

Proof. Let $T_{c}^{\prime}$ be the smallest subtree of $T$ that contains the base vertex and four ends of $d$ and $d^{\prime}$. Note that $\widetilde{\partial}_{q}(c)$ is determined by $T_{c}^{\prime}$. If both $d$ and $d^{\prime}$ have no end vertex $v$ such that $g(X, v)=\mu$, then $T_{c}^{\prime}$ does not contain the vertex $X\left(\vec{\delta}_{\mu}\right)$, and so the edge $X_{\mu}\left(\vec{\delta}_{\mu}\right)$ does not appear in the computation of $\widetilde{\partial}_{q}(c)$ since end vertices of $d$ and $d^{\prime}$ are smaller than $X\left(\vec{\delta}_{\mu}\right)$. Thus we are done.

If both $d$ and $d^{\prime}$ have end vertices $v$ such that $g(X, v)=\mu$, then the valency of $X$ in $T_{c}^{\prime}$ is two since $d$ and $d^{\prime}$ are not separated by $X\left(\vec{\delta}_{\mu}\right)$. So $\widetilde{\partial}_{q}(c)$ does not contain $X_{\mu}(\vec{x}) \cup q$ as summands. Otherwise we may assume that two ends of $d$ are on the last branch of $X$. Then $\iota(d)>\tau(d)>\iota\left(d^{\prime}\right)>\tau\left(d^{\prime}\right)$. So $\widetilde{R}\left(q \cup d \cup \iota\left(d^{\prime}\right)\right)=$ $\widetilde{R}\left(q \cup d \cup \tau\left(d^{\prime}\right)\right)$. Since $\iota(d) \wedge \iota\left(d^{\prime}\right)=\tau(d) \wedge \iota\left(d^{\prime}\right)$ and $\iota(d) \wedge \tau\left(d^{\prime}\right)=\tau(d) \wedge \tau\left(d^{\prime}\right)$, $\widetilde{R}\left(q \cup d^{\prime} \cup \iota(d)\right)=\widetilde{R}\left(q \cup d^{\prime} \cup \tau(d)\right)$. These imply $\widetilde{\partial}_{q}(c)=0$ by Lemma 4.10.

If there are deleted edges $d_{X}$ incident to $X$ in a graph $\Gamma$ containing $S_{0}$ but not $\Theta$, we need to consider critical 3-cells in $U D_{n} \Gamma$ of the form $q \cup d_{X} \cup d \cup X(\vec{x})$. From (3) of Cond $(\Gamma, i, T)$, there are no deleted edges other than $q$ separated by $X$. Thus we have the following corollary of Lemma 4.10.

Corollary 4.21. Let $\Gamma$ be a graph containing no $\Theta$ and satisfying $\operatorname{Cond}(\Gamma, i, T)$. Suppose that $c$ is a critical 3-cell of the form $q \cup d_{X} \cup d \cup X(\vec{x})$ in $U D_{n} \Gamma$. Then $\widetilde{\partial}(c)$ contains no critical 2 -cells of the form $X_{\mu}(\vec{x}) \cup q$ as a summand.

Note that only limited kinds of critical 3-cells were considered in this section, but they are sufficient in achieving our goal in the following sections where graphs should be assumed to satisfy $\operatorname{Cond}(\Gamma, i, T)$.

4.3. Homomorphisms between the second homologies. Let $\Gamma^{\prime}$ be either $S_{0}$ or $\Theta$ and $\Gamma$ be a planar graph containing $\Gamma^{\prime}$. As explained in 4.2 , we choose an embedding $i: \Gamma^{\prime} \rightarrow \Gamma$ and their maximal trees and orders satisfying $\operatorname{Cond}(\Gamma, i, T)$ so that $i$ induces a chain map $\tilde{i}$ between Morse complexes as follows:

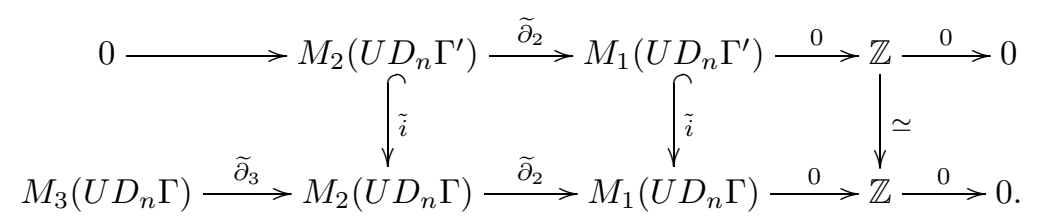

Recall the subgroup $M_{q}$ of $M_{2}\left(U D_{n} \Gamma\right)$ and the homomorphism $\widetilde{\partial}_{q}: M_{3}\left(U D_{n} \Gamma\right)$ $\rightarrow M_{q}$ defined in the previous section. When $\Gamma^{\prime}=S_{0}$, we want to prove that $\tilde{i}$ induces an injection $i_{*}: H_{2}\left(U D_{n} \Gamma^{\prime}\right) \rightarrow H_{2}\left(U D_{n} \Gamma\right)$ by showing a sufficient condition that $\tilde{i}\left(M_{2}\left(U D_{n} \Gamma^{\prime}\right)\right) \cap \widetilde{\partial}\left(M_{3}\left(U D_{n} \Gamma\right)\right)=\{0\}$. 
When $\Gamma^{\prime}=\Theta$, we want to prove that $i_{*}: H_{2}\left(U D_{n} \Gamma^{\prime}\right) \rightarrow H_{2}\left(U D_{n} \Gamma\right)$ is a nontrivial homomorphism by showing that there is a cycle $z$ in $M_{2}\left(U D_{n} \Gamma^{\prime}\right)$ and a homomorphism $\rho: M_{2}\left(U D_{n} \Gamma\right) \rightarrow \mathbb{Z}$ such that $\rho \circ \widetilde{\partial}\left(M_{3}\left(U D_{n} \Gamma\right)\right)=0$ but $\rho \circ \tilde{i}(z) \neq 0$.

Lemma 4.22. Let $\Gamma$ be a graph that contains $S_{0}$ but does not contain $\Theta$ and $n \geq 5$. Then there is an embedding $i: S_{0} \rightarrow \Gamma$ that induces an injection $i_{*}: H_{2}\left(B_{n} S_{0}\right) \rightarrow$ $H_{2}\left(B_{n} \Gamma\right)$.

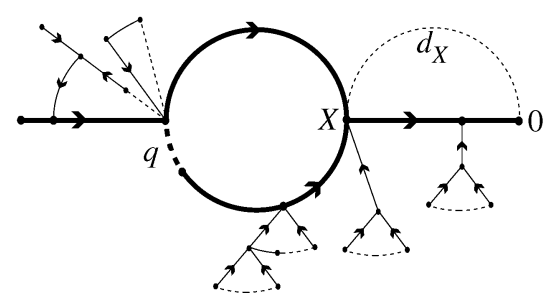

Figure 23. Maximal tree and order of graph containing $S_{0}$ but not $\Theta$

Proof. Consider an embedding $i: S_{0} \rightarrow \Gamma$ and a maximal tree $T$ of $\Gamma$ satisfying Cond $(\Gamma, i, T)$ as in Figure 23. We need to show that for each critical 3-cell $c$, $\widetilde{\partial}(c) \notin \operatorname{im}(\tilde{i})-\{0\}$. We know that $\operatorname{im}(\tilde{i})$ is generated by critical 2-cells of the form $X_{\mu}(\vec{x}) \cup q$. A critical 3-cell $c$ must contain two deleted edges including $q$. Otherwise, $\tilde{\partial}_{q}(c)=0$ by Lemma 4.12, Lemma 4.20 and Corollary 4.21 . So $c$ can be either $A_{k}(\vec{a}) \cup q \cup d$ or $A_{k}(\vec{a}) \cup q \cup d_{X} \cup X(\vec{x})$ for some vertex $A$ of valency $\geq 3$. Since $\operatorname{Cond}(\Gamma, i, T)$ implies that $d$ is not separated by $X, \widetilde{\partial}(c) \notin \operatorname{im}(\tilde{i})-\{0\}$ by Lemma 4.15 and Corollary 4.16.

Lemma 4.23. Let $\Gamma$ be a planar graph that contains $\Theta$ and $n \geq 4$. There exists an embedding $i: \Theta \rightarrow \Gamma$ such that the induced map $i_{*}: H_{2}\left(B_{n} \Theta\right) \rightarrow H_{2}\left(B_{n} \Gamma\right)$ is non-trivial.

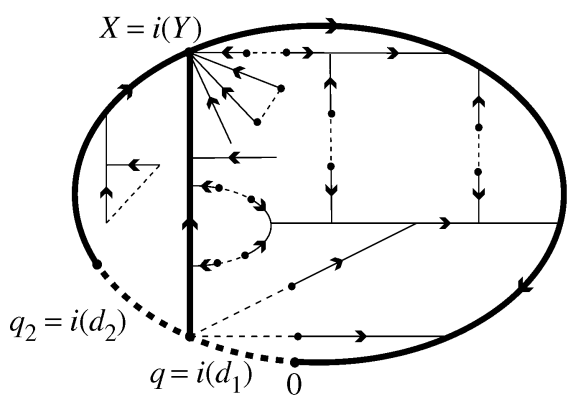

Figure 24. Planar graph containing $\Theta$

Proof. Choose an embedding $i: \Theta \rightarrow \Gamma$ and a maximal tree $T$ of $\Gamma$ such that they satisfy Cond $(\Gamma, i, T)$. Then $g\left(X, \iota\left(q_{2}\right)\right)=\mu$ and $g(X, \iota(q))=\mu-1$, where $q_{2}=i\left(d_{2}\right)$ as in Figure [24, and so $X\left(\vec{\delta}_{\mu}\right)$ separates no deleted edges. Thus the hypothesis of Lemma 4.18 and Lemma 4.20 are automatically satisfied. 
To show that $\tilde{i}_{*}$ is non-trivial, let $\alpha=\left(Y_{2}(1,2) \cup d_{1}\right)-\left(Y_{2}(2,1) \cup d_{1}\right)+\left(Y_{2}(1,1) \cup\right.$ $\left.d_{1}\right)-2\left(Y_{2}(1,1) \cup d_{2}\right)+\left(Y_{2}(2,1) \cup d_{2}\right)$, where $Y$ is the vertex and $d_{1}$ and $d_{2}$ are deleted edges in the graph $\Theta$ with the planar embedding given by Figure 16. Then $\tilde{\partial}(\alpha)=\left(2 Y_{2}(1,2)-Y_{2}(2,2)\right)-\left(Y_{2}(2,1)+Y_{2}(1,1)-Y_{2}(3,1)\right)+\left(2 Y_{2}(1,1)-Y_{2}(2,1)\right)-$ $2\left(Y_{2}(1,2)-Y_{2}(2,1)+Y_{2}(1,1)\right)+\left(Y_{2}(2,2)-Y_{2}(3,1)+Y_{2}(1,1)\right)=0$. Thus $\alpha$ is a 2 -cycle, and so $\tilde{i}_{*}(\alpha)=\left(X_{\mu}\left(\vec{\delta}_{\mu-1}+2 \vec{\delta}_{\mu}\right) \cup q\right)-\left(X_{\mu}\left(2 \vec{\delta}_{\mu-1}+\vec{\delta}_{\mu}\right) \cup q\right)+\left(X_{\mu}\left(\vec{\delta}_{\mu-1}+\right.\right.$ $\left.\left.\vec{\delta}_{\mu}\right) \cup q\right)-2\left(X_{\mu}\left(\vec{\delta}_{\mu-1}+\vec{\delta}_{\mu}\right) \cup q_{2}\right)+\left(X_{\mu}\left(2 \vec{\delta}_{\mu-1}+\vec{\delta}_{\mu}\right) \cup q_{2}\right)$ represents a homology class of $H_{2}\left(B_{n} \Gamma\right)$.

Define a homomorphism $\rho: M_{2}\left(U D_{n} \Gamma\right) \rightarrow \mathbb{Z}$ by $\rho\left(X_{\mu}(\vec{x}) \cup q\right)=x_{\mu}\left(x_{1}+\cdots+\right.$ $\left.x_{\mu-1}\right)$, and $\rho(c)=0$ for any other critical 2-cell $c$. To show $\rho$ induces a homomorphism on homologies, we need the fact that $\rho(\operatorname{im} \widetilde{\partial})$ is trivial. By the definition of $\rho$ we need to show $\rho(c)=0$ for each critical 3-cell $c$ in $M_{3}\left(U D_{n} \Gamma\right)$ such that $\widetilde{\partial}(c)$ contains a 2-cell of the form $X_{\mu}(\vec{x}) \cup q$ as a summand. By Lemma4.12, Lemma4.15. and Lemma 4.20, it suffices to consider $c=X_{\mu}(\vec{x}) \cup q \cup d$ for some $\vec{x}$ and a deleted edge $d$. Then by Lemma 4.18 ,

$$
\begin{aligned}
\widetilde{\partial}(c)= & \left(X_{\mu}\left(\vec{x}+\vec{\delta}_{g(X, \tau(d))}\right) \cup q\right)-\left(X_{\mu}\left(\vec{x}+\vec{\delta}_{g(X, \iota(d))}\right) \cup q\right) \\
& +\left(X_{\mu}\left(\vec{\delta}_{g(X, \iota(d))}+x_{\mu} \vec{\delta}_{\mu}\right) \cup q\right)-\left(X_{\mu}\left(\vec{\delta}_{g(X, \tau(d))}+x_{\mu} \vec{\delta}_{\mu}\right) \cup q\right)+\text { Irrelevant. }
\end{aligned}
$$

Then $\rho(\widetilde{\partial}(c))=0$, and so $\rho$ induces a homomorphism $\rho_{*}: H_{2}\left(M_{*}\left(U D_{n} \Gamma\right)\right) \rightarrow \mathbb{Z}$. Since $\rho\left(\tilde{i}_{*}(\alpha)\right)=1, \rho_{*}\left(\tilde{i}_{*}(\alpha)\right)$ is a non-trivial class in $H_{2}\left(M_{*}\left(U D_{n} \Gamma\right)\right)$. So the homomorphism $i_{*}: H_{2}\left(B_{n} \Theta\right) \rightarrow H_{2}\left(B_{n} \Gamma\right)$ is non-trivial.

4.4. Non-planar graphs. If we choose a maximal tree of $K_{3,3}$ and give an order as in Figure 25, then we obtain a presentation of $B_{2} K_{3,3}$ with five generators $d_{1}$, $d_{2}, d_{3}, d_{4}, B_{2}(1,1)$ and one relator $B_{2}(1,1) d_{3}^{-1} B_{2}(1,1) d_{2} d_{3} d_{1}^{-1} d_{2}^{-1} d_{4} d_{2} d_{1} d_{2}^{-1} d_{4}^{-1}$. The abelianization of the relator is $\left(B_{2}(1,1)\right)^{2}$. Thus $H_{1}\left(B_{2} K_{3,3}\right)$ has a 2-torsion, even though every graph braid group is torsion-free.

However, there are two difficulties in a straightforward extension of this argument to show that the first homology group of any non-planar graph braid group has a torsion. One is that it is quickly getting out of hand to obtain a presentation of the $n$-braid group of $K_{5}$ or $K_{3,3}$ as the braid index $n$ increases. The other is that both $K_{5}$ or $K_{3,3}$ have no vertices of valency 1 , and so there is no room to accommodate extra punctures when we construct an embedding to obtain corresponding results for larger braid indices. Instead of computing the whole $H_{1}\left(B_{n} \Gamma\right)$, we will find a critical 1-cell that produces a non-trivial torsion homology class. Lemma 4.8 is useful to show it is in fact a 2-torsion. The homomorphism $\pi_{*}: H_{1}\left(B_{n} \Gamma\right) \rightarrow \mathbb{Z}_{2}(=$ $H_{1}\left(S_{n}\right)$ ) discussed after Example 2.3 is used to check that it is non-trivial.

Let a graph $\Gamma$ be ordered by a planar embedding of its maximal tree $T$ satisfying the fact that both ends of every deleted edge have valency two in $\Gamma$. Suppose the vertex $i$ in $0_{n}$ is regarded as a puncture labeled by $n-i$ for $0 \leq i \leq n-1$. If neither ends of a deleted edge $d$ are the base vertex $0, \pi(d)$ is the identity, and so $\pi_{*}([d])=0$. If one of the ends of $d$ is the base vertex $0, \pi(d)=(1, n, n-1, \ldots, 2)$, and so $\pi_{*}([d]) \equiv n-1(\bmod 2)$. For a critical 1-cell $A_{k}(\vec{a}), \pi\left(A_{k}(\vec{a})\right)=\left(|\vec{a}|,|\vec{a}|-1, \ldots,|\vec{a}|_{k}\right)$, and so

$$
\pi_{*}\left(\left[A_{k}(\vec{a})\right]\right) \equiv|\vec{a}|-|\vec{a}|_{k} \quad(\bmod 2),
$$

where $|\vec{a}|_{k}=a_{k}+a_{k+1}+\cdots+a_{m}$ for $\vec{a}=\left(a_{1}, \ldots, a_{m}\right)$. 
Lemma 4.24. For the complete bipartite graph $K_{3,3}$ of 6 vertices and for $n \geq 2$, $H_{1}\left(B_{n} K_{3,3}\right)$ has a torsion.

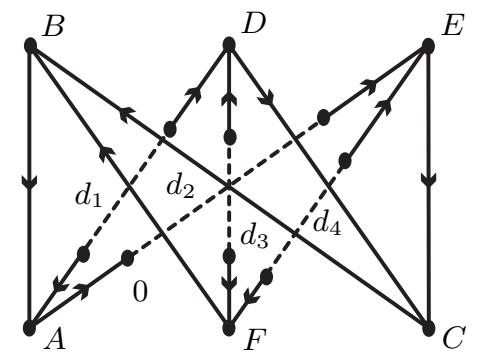

Figure 25. Maximal tree and order of $K_{3,3}$

Proof. If we choose a maximal tree of $K_{3,3}$ and its planar embedding as in Figure 25. then the complex $U D_{n} K_{3,3}$ has critical 1-cells $B_{2}(1,1), C_{2}(1,1)$ and critical 2-cells $d_{1} \cup d_{4}, d_{2} \cup d_{3}$. Using Lemma 4.8 and the maximal tree, the boundaries of critical 2-cells are given by

$$
\widetilde{\partial}\left(d_{1} \cup d_{4}\right)=B_{2}(1,1)-C_{2}(1,1), \quad \widetilde{\partial}\left(d_{2} \cup d_{3}\right)=B_{2}(1,1)+C_{2}(1,1) .
$$

Thus $2 B_{2}(1,1)$ is a boundary, and so it represents 0 in $H_{1}\left(M_{*}\left(U D_{n} K_{3,3}\right)\right)$. However, $\pi_{*}\left(\left[B_{2}(1,1)\right]\right)=1$, so $B_{2}(1,1)$ represents a non-trivial torsion class in $H_{1}\left(B_{n} K_{3,3}\right)$.

Lemma 4.25. For the complete graph $K_{5}$ of 5 vertices and for $n \geq 2, H_{1}\left(B_{n} K_{5}\right)$ has a torsion.

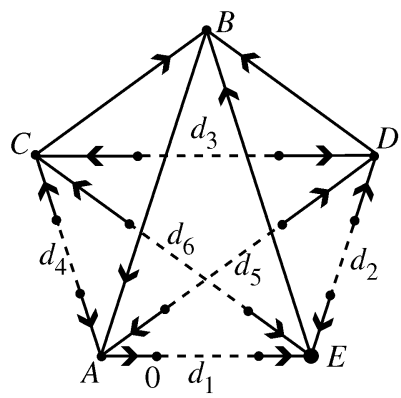

FiguRE 26. Maximal tree and order of $K_{5}$

Proof. If we choose a maximal tree of $K_{5}$ and a planar embedding as in Figure 26, then $U D_{n} K_{5}$ has critical 1-cells $B_{2}(1,1,0), B_{3}(1,0,1), B_{3}(0,1,1)$ and critical 2cells $d_{1} \cup d_{3}, d_{2} \cup d_{4}, d_{5} \cup d_{6}$. Using Lemma 4.8 and the maximal tree, the boundaries of critical 2-cells are given by $\widetilde{\partial}_{2}\left(d_{1} \cup d_{3}\right)=B_{3}(1,0,1)-B_{3}(0,1,1)$, $\widetilde{\partial}_{2}\left(d_{5} \cup d_{6}\right)=B_{2}(1,1,0)+B_{3}(0,1,1), \widetilde{\partial}_{2}\left(d_{2} \cup d_{4}\right)=B_{3}(1,0,1)-B_{2}(1,1,0)$. Thus $2 B_{2}(1,1,0)$ is a boundary and represents the trivial class in $H_{1}\left(M_{*}\left(U D_{n} K_{5}\right)\right)$. But $\pi_{*}\left(\left[B_{2}(1,1,0)\right]\right)=1$, and so it represents a torsion class. So $H_{1}\left(B_{n} K_{3,3}\right)$ has a torsion. 
Lemma 4.26. For any non-planar graph $\Gamma$ and for $n \geq 2, H_{1}\left(B_{n} \Gamma\right)$ has a torsion, and so $B_{n} \Gamma$ is not a right-angled Artin group.

Proof. Since every non-planar graph contains $K_{5}$ or $K_{3,3}$, there is an embedding $i: K \rightarrow \Gamma$ where $K$ is $K_{5}$ or $K_{3,3}$. We choose a maximal tree $T_{K}$ and its planar embedding as in Figure 25] or 26 and choose a maximal tree $T$ of $\Gamma$ satisfying the following conditions:

(1) Both ends of each deleted edge have valency two in $\Gamma$.

(2) The base vertex of $K$ maps to the base vertex on $\Gamma$ under $i$.

(3) For an edge of $K, i(e)$ is a deleted edge of $\Gamma$ if and only if $e$ is a deleted edge of $K$.

(4) $i$ is order preserving.

We note that conditions (3) and (4) automatically hold if $T$ contains $T_{K}$ as a subgraph in the plane. Under these conditions, it is not hard to see that the embedding $i$ preserves critical cells and boundaries of critical cells, that is, $i$ induces a chain map on Morse chain complexes. Thus it induces a homomorphism $i_{*}$ : $H_{*}\left(M_{*}\left(U D_{n} K\right)\right) \rightarrow H_{*}\left(M_{*}\left(U D_{n} \Gamma\right)\right)$.

Let $\left[B_{2}(\vec{a})\right]$ be a torsion class in $H_{1}\left(M_{*}\left(U D_{n} K\right)\right)$ as given in Lemma 4.24 or Lemma 4.25. Then $\pi_{*}\left(i_{*}\left(\left[B_{2}(\vec{a})\right]\right)\right)=1$, and so $i_{*}\left(\left[B_{2}(\vec{a})\right]\right)$ is a torsion class in $H_{1}\left(M_{*}\left(U D_{n} \Gamma\right)\right)$.

\section{Conjectures}

We finish the article with a few observations and conjectures.

An $n$-nucleus is a minimal graph whose $n$-braid group is not a right-angled Artin group, that is, the $n$-braid group of an $n$-nucleus is not a right-angled Artin group and the $n$-braid group of any graph contained in an $n$-nucleus is a right-angled Artin group. Our main result says that for $n \geq 5$, a graph $\Gamma$ contains no $n$-nuclei if and only if $B_{n} \Gamma$ is a right-angled Artin group and that there are two $n$-nuclei $T_{0}$ and $S_{0}$. It is natural to expect that the corresponding statement works for all braid indices, and so we first propose

Conjecture 5.1. For any braid index $n \geq 2$, a graph $\Gamma$ contains no $n$-nuclei if and only if $B_{n} \Gamma$ is a right-angled Artin group.

Then we propose a complete list of 4-nuclei and 3-nuclei as follows:

Conjecture 5.2. There are four 4-nuclei as given in Figure 27 and ten 3-nuclei as given in Figure 28.

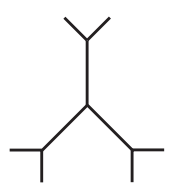

(a)

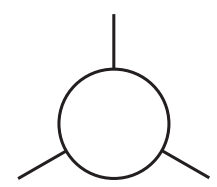

(b)

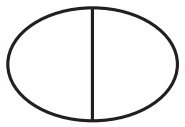

(c)

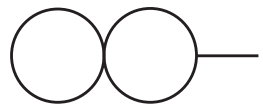

(d)

FigURE 27. 4-Nuclei

We found more than sixty 2-nuclei as given in Figure 29. Due to their plethora, only representatives from each type of 2-nuclei are listed, and each type contains as many graphs as the number in parentheses. 


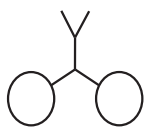

(a)

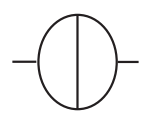

(b)

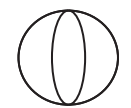

(c)

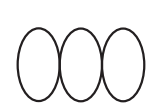

(d)

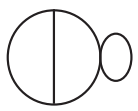

(e)

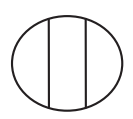

(f)

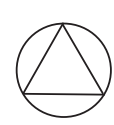

(g)

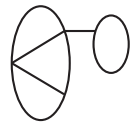

(h)

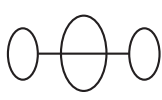

(i)

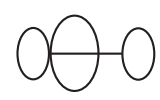

(j)

FiguRE 28. 3-Nuclei

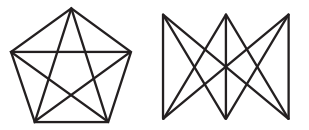

(a) Non-planar (2)

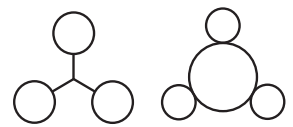

(b) Type 1 (5)

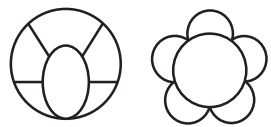

(c) Type 2 (7)

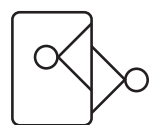

(d) Type 3 (17)

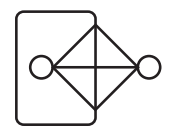

Figure 29. 2-Nuclei by types
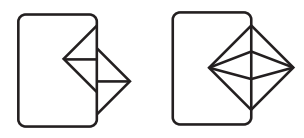

(e) Type $4(\geq 30)$

Given a planar graph, the presentation of its $n$-braid group obtained by choosing a maximal tree as explained in 2.2 seems relatively nice in the sense that there is a systematic application of Tietze transformations to make it a commutator-ralated group. We state this as a conjecture:

Conjecture 5.3. Let $\Gamma$ be a planar graph and $n \geq 2$. Then $B_{n} \Gamma$ is a commutatorralated group.

In 4.4 we show that if a given graph is non-planar, the first homology of its braid group has a 2-torsion. The converse is a corollary of the previous conjecture, and so we propose

Conjecture 5.4. For $n \geq 2$, a graph $\Gamma$ is planar if and only if $H_{1}\left(B_{n} \Gamma\right)$ is torsionfree.

We can prove the above conjecture for braid index $n=2$ by using Lemma 4.8 as follows.

Theorem 5.5. A graph $\Gamma$ is planar if and only if $H_{1}\left(B_{2} \Gamma\right)$ is torsion-free.

Proof. It only remains to prove that if a graph is planar, then $H_{1}\left(B_{2} \Gamma\right)$ is torsionfree. We first assume that each path between two vertices of valency $\neq 2$ or each simple loop in $\Gamma$ passes through exactly 3 edges. We choose the maximal tree $T$ of $\Gamma$ and give an order on vertices so that for each deleted edge $d$, both $\iota(d)$ and $\tau(d)$ are of valency 2 and $\iota(d) \wedge \tau(d)$ is always the nearest vertex of valency $\geq 3$ from $\tau(d)$ unless $\tau(d)=0$. This can be achieved by the following steps:

(I) Choose a planar embedding of $\Gamma$, and choose a vertex of valency 1 as a base vertex 0 if there is one. Let $T=\Gamma$. If there are no vertices of valency 1 , 
choose a vertex of valency 2 as a base vertex 0 and delete the edge, one of whose end vertices is 0 , and let $T$ be the rest of $\Gamma$. Proceed to (II).

(II) Take a regular neighborhood $R$ of $T$. As traveling the outmost component of $\partial R$ clockwise from the vertex numbered the last, number the unnumbered vertices of $T$ until either coming back to 0 or numbering a vertex of valency 2 that belongs to a circuit in $T$. If the former is the case, we are done. If the latter is the case, delete the edge incident to the vertex in front and let $T$ be the rest. Repeat (II).

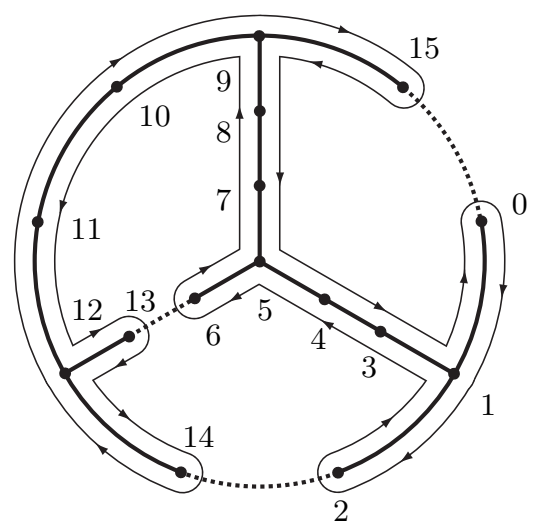

FIGURE 30. Numbering a maximal tree $T$ of $K_{4}$

Then it is clear that we have the property that

(*) for each deleted edge $d$, there are no vertices of valency $\geq 3$ between $\tau(d)$ and $\iota(d) \wedge \tau(d)$ in $T$.

A 2-cell cannot contain any non-order-respecting edge in $U D_{2} \Gamma$, and so there is only one kind of critical 2-cell of the form $d \cup d^{\prime}$ for deleted edges $d, d^{\prime}$.

To classify these critical 2-cells $c$ and to compute their images under $\widetilde{\partial}$, we consider the smallest subtree $T_{c}$ of the maximal tree $T$ that contains four ends of $d$ and $d^{\prime}$ together with the base vertex. By condition $(*)$, there are eight possibilities for $T_{c}$ given by Figure 31(a)-(h) if none of the four ends is the base vertex. Also, there are three possibilities for $T_{c}$ given by Figure 31(i)-(k) if one of the four ends is the base vertex. We may consider each possible $T_{c}$ together with the formulae for $\widetilde{\partial}_{2}\left(d \cup d^{\prime}\right)$ in Lemma 4.8. Note that the order of $T_{c}$ is given as in Step I of $\$ 2.2$.

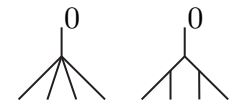

(a) (b)

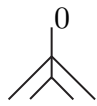

(c)

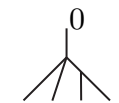

(d)

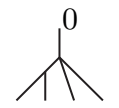

(e)

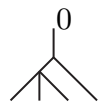

(f)
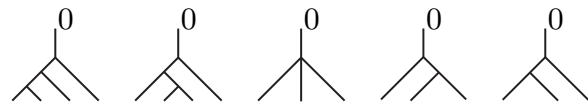

(g)

(h)

(i)

(j)

(k)

Figure 31 . Possible $T_{c}$ 
Since $\Gamma$ is planar, we do not have case (3) of Lemma 4.8. The critical 2-cell satisfying Lemma $4.8(\ell)$ for $\ell=1,2$ and whose four end vertices form the subtree of $T$ given in Figure $31(\alpha)$ for $\alpha=a, \ldots, k$ is denoted by $c_{\ell \alpha}$. For example,

$$
\begin{aligned}
\widetilde{\partial}\left(c_{1 a}\right)= & A_{g\left(A, \tau\left(d^{\prime}\right)\right)}\left(\vec{\delta}_{g\left(A, \tau\left(d^{\prime}\right)\right)}+\vec{\delta}_{g(A, \tau(d))}\right)-A_{g\left(A, \tau\left(d^{\prime}\right)\right)}\left(\vec{\delta}_{g\left(A, \tau\left(d^{\prime}\right)\right)}+\vec{\delta}_{g(A, \iota(d))}\right) \\
& -A_{g\left(A, \iota\left(d^{\prime}\right)\right)}\left(\vec{\delta}_{g\left(A, \iota\left(d^{\prime}\right)\right)}+\vec{\delta}_{g(A, \tau(d))}\right)+A_{g\left(A, \iota\left(d^{\prime}\right)\right)}\left(\vec{\delta}_{g\left(A, \iota\left(d^{\prime}\right)\right)}+\vec{\delta}_{g(A, \iota(d))}\right),
\end{aligned}
$$

where $A=\iota(d) \wedge \tau(d)=\iota\left(d^{\prime}\right) \wedge \tau\left(d^{\prime}\right)$. It is easy to see that every critical 2-cell except the five kinds of critical 2-cells $c_{1 a}, c_{1 c}, c_{2 a}, c_{2 d}$, and $c_{2 e}$ gives either the trivial relation or a relation which is the difference between two critical 1-cells. By condition $(*), c_{1 c}$ and $c_{2 d}$ cannot occur. For each of the other three cases, its

boundary contains $B_{g\left(B, \tau\left(d^{\prime}\right)\right)}\left(\vec{\delta}_{g\left(B, \tau\left(d^{\prime}\right)\right)}+\vec{\delta}_{g(B, \tau(d))}\right)$ as a summand which does not appear in the boundary of any other critical 2-cells where $B=\tau(d) \wedge \tau\left(d^{\prime}\right)$.

Consequently, we have a presentation matrix of $H_{1}\left(B_{2} \Gamma\right)$ whose rows satisfy one of the following:

(i) consisting of all zeros;

(ii) consisting of zeros except a pair of entries 1 and -1 ;

(iii) containing 1 that is the only non-zero entry in the column in which it belongs.

Then it is easy to see via elementary row and column operations that this implies that $H_{1}\left(B_{2} \Gamma\right)$ is torsion-free.

\section{REFERENCES}

[1] A. Abrams, Configuration space of braid groups of graphs, Ph.D. thesis in UC Berkeley, ProQuest LLC, Ann Arbor, MI, 2000. MR2701024

[2] A. Abrams and R. Ghrist, Finding topology in factory: Configuration spaces, Amer. Math. Monthly 109(2) (2002), 140-150. MR.1903151 (2003c:68222)

[3] W. Bruns and J. Gubeladze, Combinatorial invariance of Stanley-Reisner rings, Georgian Math. J. 3(4) (1996), 315-318. MR1397814 (97d:13025)

[4] R. Charney, An introduction to right-angled Artin groups, Geom. Dedicate 125 (2007), 141158. MR2322545 (2008f:20076)

[5] R. Charney and M. Davis, Finite $K(\pi, 1)$ s for Artin groups, Prospects in topology, Ann. of Math. Stud. 138, Princeton Univ. Press, Princeton, NJ, 1995, pp. 110-124. MR.1368655 (97a:57001)

[6] F. Connelly and M. Doig, Braid groups and right-angled Artin groups, arXiv:math.GT/0411368.

[7] J. Crisp and B. Wiest, Embeddings of graph braid and surface groups in right-angled Artin groups and braid groups, Algebr. Geom. Topol. 4 (2004), 439-472. MR2077673(2005e:20052)

[8] D. Farley, Homology of tree braid groups, Contemp. Math. 394 (2006), 102-112. MR2216709 (2007c:20093)

[9] D. Farley and L. Sabalka, Discrete Morse theory and graph braid groups, Algebr. Geom. Topol. 5 (2005), 1075-1109 (electronic). MR 2171804 (2006f:20051)

[10] D. Farley and L. Sabalka, On the cohomology rings of tree braid groups, J. Pure Appl. Algebra 212(1) (2007), 53-71. MR2355034 (2008j:20107)

[11] R. Forman, Morse theory for cell complexes, Adv. Math. 143(1) (1998), 90-145. MR.1612391 (99b:57050)

[12] R. Ghrist, Configuration spaces and braid groups on graphs in robotics, Knots, braids, and mapping class groups-papers dedicated to Joan S. Birman (New York, 1998), pp. 2940, AMS/IP Stud. Adv. Math. 24, Amer. Math. Soc., Providence, RI, 2001. MR.1873106 (2002j:55015) 
[13] D. Matei and A. Suciu, Cohomology rings and nilpotent quotients of real and complex arrangements, Arrangements-Tokyo 1998, Adv. Stud. Pure Math. 27, Kinokuniya, Tokyo, 2000, pp. 185-215. MR1796900 (2002b:32045)

[14] L. Sabalka, Embedding right-angled Artin groups into graph braid groups, Geom. Dedicata 124 (2007), 191-198. MR 2318544 (2008e:20057)

Department of Mathematics, Korea Advanced Institute of Science and Technology, DAEJEON, 305-701, KoreA

E-mail address: kimjeehyoun@kaist.ac.kr

Department of Mathematics, Korea Advanced Institute of Science and Technology, DAEJEON, 305-701, KOREA

E-mail address: knot@kaist.ac.kr

Department of Mathematics, Korea Advanced Institute of Science and Technology, DAejeon, 305-701, Korea

E-mail address: H.W.Park@kaist.ac.kr 